\title{
Mobile Phone Technologies and their Impacts on Household Welfare and Rural Development in Uganda
}

Dissertation

to obtain the Ph.D. degree

in the International Ph. D. Program for Agricultural Sciences in Göttingen (IPAG) at the Faculty of Agricultural Sciences,

Georg-August-University Göttingen, Germany

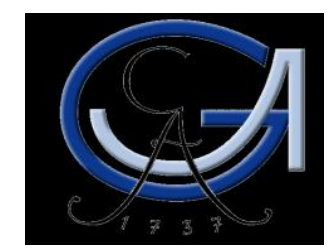

presented by

Haruna Ahmad Sekabira

born in Kampala, Uganda

Göttingen, $26^{\text {th }}$ July 2017 
1. Name of supervisor: Prof. Dr. Matin Qaim

2. Name of co-supervisor: Prof. Xiaohua $\mathrm{Yu}, \mathrm{PhD}$

3. Name of co-supervisor: Prof. Dr. Stephan von Cramon-Taubadel

Date of dissertation: 11 May, 2017 


\section{Summary}

In the past two decades, since around 1995, mobile phone (MP) technologies have been widely adopted in developing countries - with the highest penetration rates in Sub-Saharan Africa, to levels of about $89 \%$ for adults. Previous research has shown MP use to enhance market access through information exchange and market price integration - availing timely updates on prices of inputs and outputs. Various applications of MP technologies, for instance mobile money (MM) services, where money is transferred electronically between the sender and the receiver using mobile phones, have also cropped up and are widely predicted to have life-enhancing effects for rural households. More specifically, the available literature has shown that MM services can contribute to welfare gains in smallholder farm households via several pathways. One important pathway for MM-related welfare gains are higher remittances received by MM users from relatives and friends.

However, the impact of MP use and many of its key applications, like MM services, on several smallholder welfare aspects has barely been investigated. In particular, we are not aware of any studies that have analyzed the effects of MP use on gender equality and nutrition - two welfare dimensions that are of particular importance in the context of the United Nations' Sustainable Development Goals (SDGs), that aim at ending hunger, achieving food security and improved nutrition, as well as attaining gender equality and women empowerment. Furthermore, there is yet no study examining the role of MM services and its several impact pathways on household welfare. These are significant gaps in the literature given that mobile phone technologies are so widely adopted among rural and urban households in the developing world. The impacts of MP technologies are predicted to be higher in developing countries than in the developed world, 
given that the infrastructure for other communication technologies and related services is much less developed in the developing world.

We address these research gaps by using panel data from smallholder farm households in Uganda. Specifically, we examine the impact of MP use on household incomes, gender equality, and nutrition. Furthermore, the impact of MM services on household welfare and impact pathways are examined, especially focusing on agricultural marketing and off-farm economic activities.

Using panel regression models, we find that MP use has positive influence on household income, gender equality, and dietary diversity. Gender-disaggregated data analysis shows that female MP use bears stronger influences on household incomes, gender equality, and nutrition than male MP use. Using simultaneous equations, we establish that female MP use's positive nutrition effects are channeled through increased incomes and gender equality. These effects are due to lower transaction costs and better access to information through MP use.

Furthermore, regression models show that the adoption of MM technology has contributed to higher household incomes and consumption levels. Off-farm income gains are also identified to be an important pathway through which MM services enhance household income, even when excluding remittances from the calculation of off-farm income. Other off-farm income sources include small businesses in trade (like retail shops, sale of forest products), transport (like motor cycle riding services for transportation of goods or humans), and handicrafts (like brick laying, mats making, clothes sewing, and carpentry services). These economic activities benefit from novel savings and money transfer opportunities through MM services. In terms of agricultural marketing, MM users sell a larger proportion of their coffee as shelled beans (a high value form 
of coffee, sold after processing) to buyers in high-value markets, instead of selling to local traders immediately after harvest. MM services also help to reduce cash constraints and facilitate quick and reliable transactions with buyers from outside local regions.

We conclude that the use of MP technologies contributes to a broader inclusive and comprehensive rural development and poverty reduction, encompassing improved household income, food security, and gender equality. We also conclude that MM services can contribute to rural development through various important pathways - especially enhancing volumes of offfarm incomes earned by rural households. The observed adoption patterns suggest that MM services are socially inclusive. In terms of policy recommendations, we conclude that gendersensitive dissemination policies for mobile phones and related technologies could broaden household income and nutrition welfare effects. 


\section{Acknowledgements}

First, I am thankful to God for the health and wisdom granted to me during the demanding period of writing this dissertation. Then, I acknowledge my gratefulness towards many persons and organizations that made this dissertation possible. Primarily, I am sincerely grateful to my main supervisor, Prof. Dr. Matin Qaim who worked with me since 2011 as I developed my research proposal for submission to the German Academic Exchange Service (DAAD). I am especially grateful for his effort and coordination with Makerere University to enable access to my Master's degree results required for my admission to the University of Goettingen. He has also continuously guided me technically throughout the writing of all papers. I am sincerely grateful to all his guidance, support, and inspiration. I also thank and appreciate Prof. Xiaohua $\mathrm{Yu}, \mathrm{PhD}$, and Prof. Dr. Stephan von Cramon-Taubadel, for accepting to co-supervise this dissertation, and be part of the examination committee. Their comments during doctoral seminars as discussants helped shape the work better.

My colleagues and fellow doctoral students at the GlobalFood Research Training Group (RTG 1666) were also very supportive and useful with good comments. I am appreciative to Eva Meemken, Kathrin Demmler, and Kerstin Grosch who were my discussants for my three mandatory doctoral seminars. More specially, I am thankful to Eva Meemken who provided a very good work relationship, support, and guidance during the three months of data collection in Masaka and Luwero. I also appreciate all my other fellow doctoral students for both their informal and formal relationships and collaborations that made my stay in Goettingen more enjoyable, to facilitate the completion of this dissertation. I am also thankful to Brian Chiputwa who collected the first round of the data used in this dissertation. I am also thankful to fellow doctoral students at the Chair of International Food Economics and Rural Development for their support and the interactions we had. Our enumerators Yusuf Kaweesa, George Jjaggwe, Nashiba Nakabira, Mariam Ssekabira, Sarah Mugerwa, Mariam Nalumansi, and Lydia Nnamakula, as well as our local guides, and host institution Kibinge coffee, are 
also appreciated. More importantly, I am thankful to all survey respondents and their families who participated in our interviews. All those who supported our work are also much appreciated.

This research was financially supported by the German Research Foundation (Deutsche Forschungsgemeinschaft, DFG) as part of the GlobalFood RTG. My stipend for the three years spent in Germany was provided by the German Academic Exchange Service (DAAD). This financial support is thankfully acknowledged.

Finally, my family is much appreciated for all visible and invisible support rendered to me to make this work complete. First, I appreciate all efforts by my late mother - God be pleased with her; Jamiida (Jamiilah) Nabisubi for the good soft meaningful guidance that she always rendered, which shaped my intelligence, patience, and in depth understanding of situations. My father, Diriisa Walusimbi is also much appreciated for his vision to keep supporting us financially and mentally towards pursuing our studies with excellence. My brother Yusuf Kaweesa, is also much appreciated for his support, guidance and back-up driving support during field work, and making my colleague Eva Meemken comfortable during field visits, as well as leading our field teams successfully.

More finally, my nuclear family is so much appreciated as this made my stay in Germany more comfortable and possible. My wife, Zainab Nansubuga, my son, Haruna Sekabira, and my daughter, Jamiilah Nabisubi are gratefully appreciated in this regard, for their love, prayers, comfort, guidance, smiles, support, and maintenance of hope. My maternal grandmother, Joweria Nazziwa, and late paternal grandfather, Yusuf Kaweesa Katanyooleka, are also appreciated for their guidance.

Most finally, my special friend Shamim Nalunga Lugoloobi is also dearly appreciated for her support, guidance, comfort, fun times, and continued encouragement.

This dissertation is dedicated to my late mother, may God ever be pleased with her. 


\section{Table of Contents}

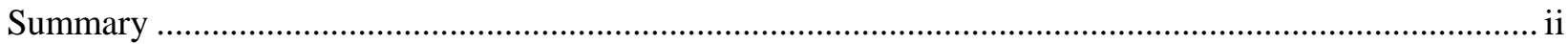

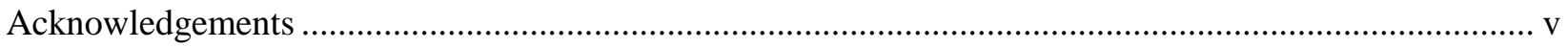

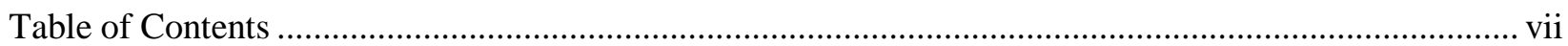

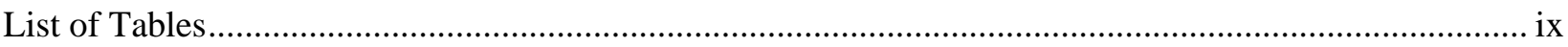

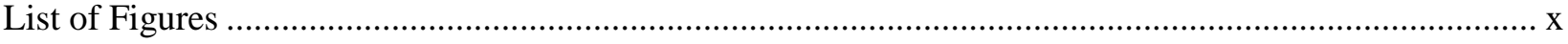

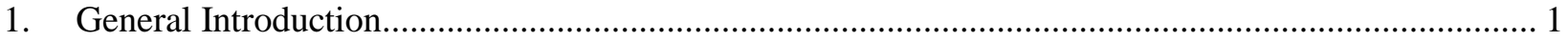

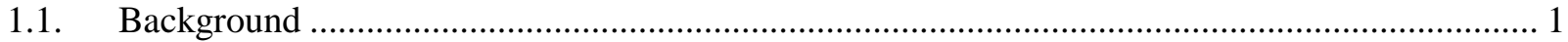

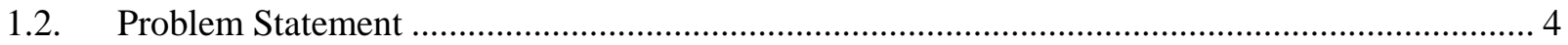

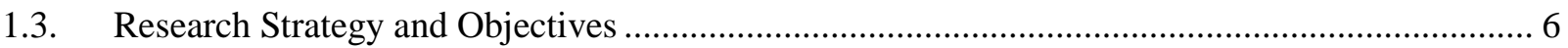

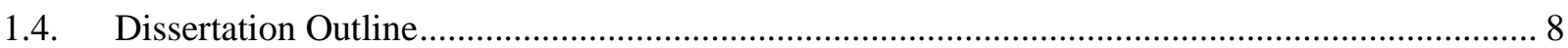

2. Can Mobile Phones Improve Gender Equality And Nutrition? Panel Data Evidence from Farm

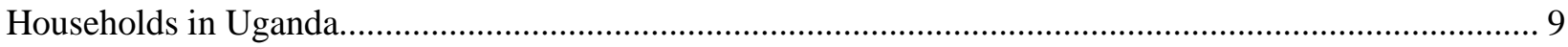

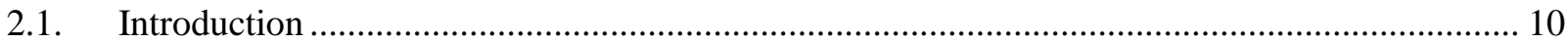

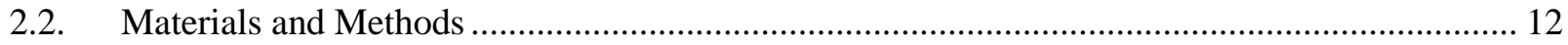

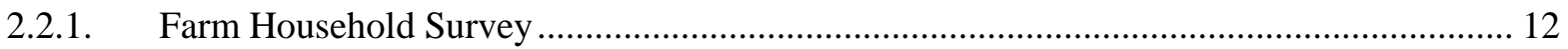

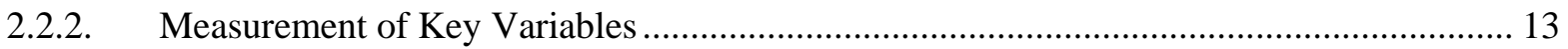

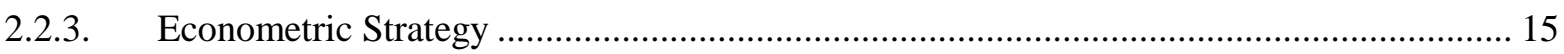

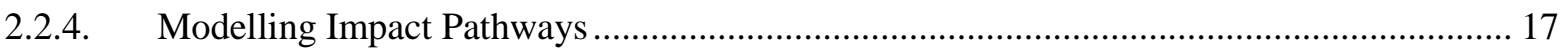

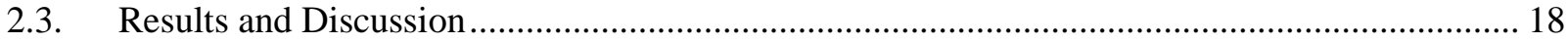

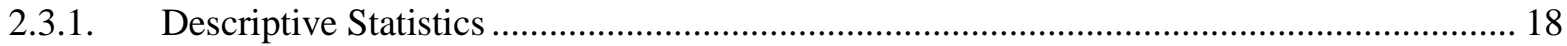

2.3.2. Aggregate Effects of Mobile Phone Use ......................................................................... 21

2.3.3. Gender Disaggregated Effects of Mobile Phone use ....................................................... 24

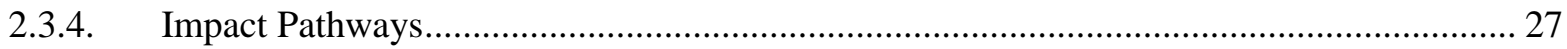

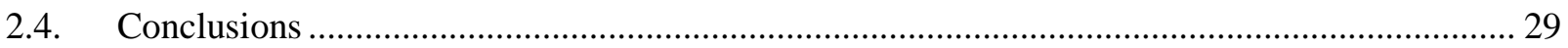

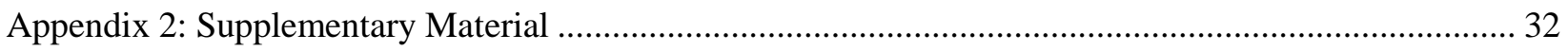

3. Mobile Money, Agricultural Marketing, and Off-Farm Income in Uganda.................................... 35

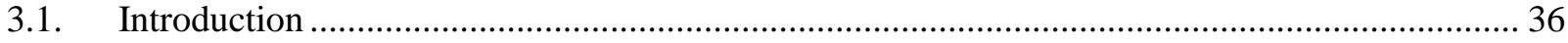

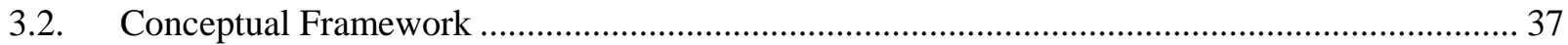

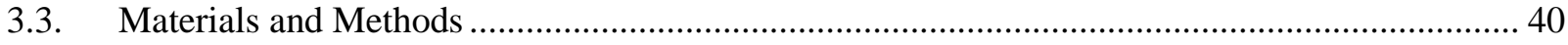




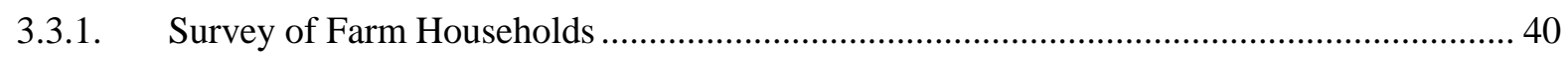

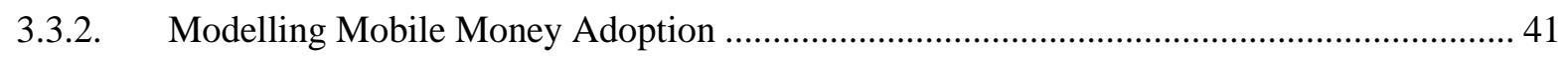

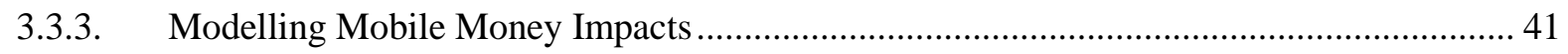

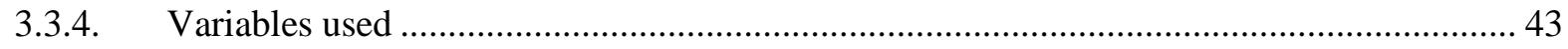

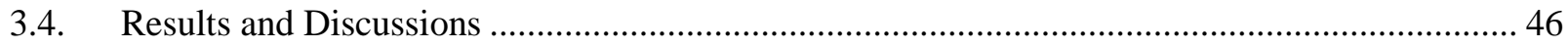

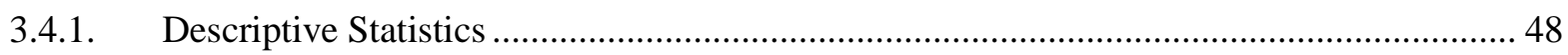

3.4.2. Determinants of Mobile Money Adoption ........................................................................ 50

3.4.3. Impact of Mobile Money Use on Household Welfare ..................................................... 53

3.4.4. Impact of Mobile Money Use on Remittances and Off-Farm Income .............................. 54

3.4.5. Impact of Mobile Money Use on Agricultural Marketing ............................................... 56

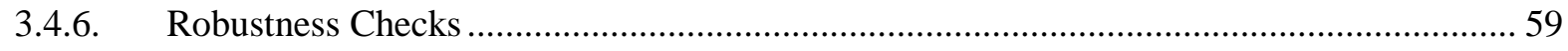

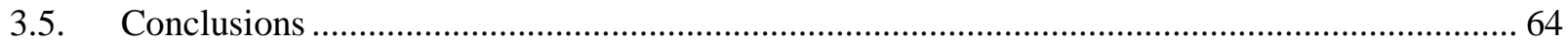

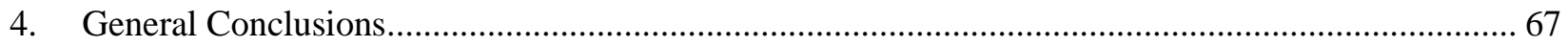

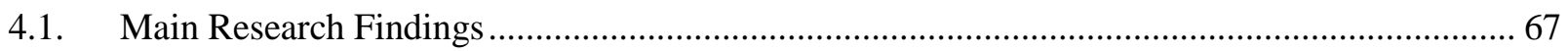

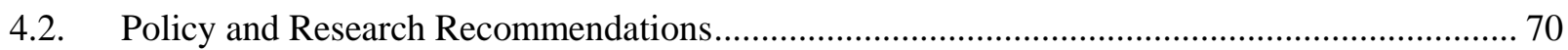

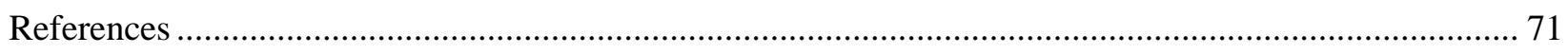

General Appendix (Household Survey Questionnaire - Uganda 2015) .............................................. 80 


\section{List of Tables}

Table 2.1: Number of households in the sample using and not using mobile phones............................... 19

Table 2.2: Socioeconomic characteristics by mobile phone use ........................................................... 19

Table 2.3: Household income, gender equality, and nutrition by mobile phone use ............................... 20

Table 2.4: Effects of mobile phone use on household income and gender equality ................................ 22

Table 2.5: Effects of mobile phone use on household nutrition ............................................................ 23

Table 2.6: Effects of female and male mobile phone use on household income and gender equality ........ 25

Table 2.7: Effects of female and male mobile phone use on household nutrition..................................... 26

Table 2.8: Effects of female mobile phone use (summary of causal pathways) ...................................... 27

Table 2.9: Socioeconomic characteristics by female mobile phone use (FMP) ....................................... 32

Table 2.10: Socioeconomic characteristics by male mobile phone use (MMP) ...................................... 33

Table 2.11: Effects of female mobile phone use (causal pathways) ....................................................... 34

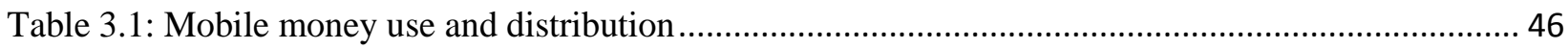

Table 3.2: Descriptive statistics by users and non-users of mobile money ............................................ 48

Table 3.3: Determinants of mobile money adoption .......................................................................... 51

Table 3.4: Impact of mobile money use on household welfare .............................................................. 53

Table 3.5: Impact of mobile money use on remittances and off-farm income........................................ 55

Table 3.6: Impact of mobile money use on proportion of coffee sold as shelled beans............................. 57

Table 3.7: Impact of mobile money use on coffee prices received ......................................................... 58

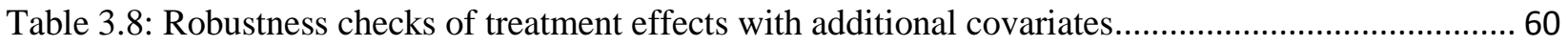

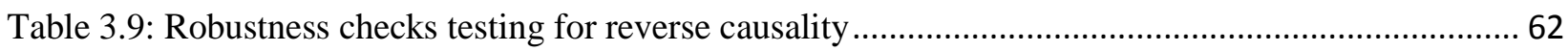




\section{List of Figures}

Figure 3.1: Impact pathways of mobile money use on household welfare ............................................ 38

Figure 3.2: Most important mobile money activity .............................................................................. 47

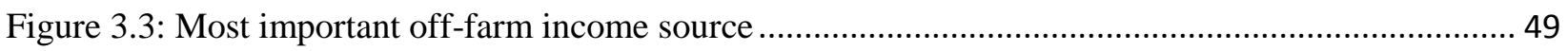




\section{General Introduction}

\subsection{Background}

Mobile phone (MP) technologies have become an important tool in facilitating markets' access thus promoting social and economic development (Jussawalla 1999; Scott et al. 2004; Torero and Von Braun 2005; Blattman, Jensen and Roman 2002; Aker and Mbiti 2010; Nakasone, Torero and Minten 2014). Mobile phones currently enable economic and social inclusiveness for populations that are deprived by inadequate infrastructure. Such deprivation has been more severe for rural areas that are inhabited by three quarters of the worlds' humanity. Through reducing negative effects of such market access and information deprivation; mobile phones have showed hope for sustainable and inclusive positive economic and social development impacts (Jensen 2010; Von Braun 2010; Aker and Mbiti 2010; Nakasone et al. 2014).

Mobile phones have been instrumental in Africa and Asia, used by populations to access information on key sectors for instance; education, health, housing, employment, transport, and markets. Mobile phones also enable cashless mobile money transfers, that would otherwise be difficult or impossible (Chowdhury, Negassa and Torero 2005; Jensen 2007 and 2010; Bhavnani et al, 2008; Aker and Mbiti, 2010; Sekabira, Bonabana and Asingwire 2012; Hoddinott, Rosegrant and Torero, 2013). More specifically, money transfer services via mobile phones enables smoothing of consumption in the mentioned sectors, through enabling access to cash money. Access to cash enables households pay for services like food, housing, education, medication, farm inputs, and others that are important for household consumption. Therefore mobile phones help the unbanked rural/urban poor access financial services, food aid, and 
business opportunities' security (Aker and Mbiti 2010; UNCTAD 2012; UCC 2013; Kirui et al. 2013; Kikulwe, Fischer and Qaim 2014 and GSMA 2014).

Despite such hypothesized importance of mobile phones highlighted in literature, there has been limited empirical evidence documenting the importance of mobile phones, and its key applications. More specifically, empirical research on economic welfare impacts of mobile phones on smallholder households is currently limited to prices (Jensen 2007; Aker, 2010), market participation (Muto and Yamato 2009) and social networks (Yamato 2012). Similarly, there is limited empirical evidence on welfare impacts of mobile money services on smallholder households. Current coverage of empirical evidence with regards to mobile money services impacts on smallholder households covers crop commercialization, input use, and incomes (Kirui et al. 2013 and Kikulwe et al. 2014). Yet, mobile phones, and its applications have broader social and economic welfare effects beyond prices, incomes, and information access, (Aker and Mbiti 2010; Nakasone et al. 2014; World Bank (2014).

In certain key areas of particular importance as regards the United Nation's Sustainable Development Goals (SDGs) like nutrition, and gender equality; these impacts have not been empirically investigated at all, constituting substantial empirical research gaps that this dissertation sought to bridge. Mobile phones can improve nutrition and gender equality through enabling awareness on nutrition and gender equality. Other established effects of mobile phones like improved household incomes, can also empower various gender economically thus enhancing gender equality. Improved incomes via mobile phone use can as well smooth household consumption, hence improving food security and dietary quality hence household 
nutrition. Based on gender of the household member that controls incomes from the use of mobile phones; such control can as well impact on household nutrition.

As motivation however, some studies on mobile phones' use have been done and established positive influence on a number of aspects for instance; global entrepreneurship (West 2012), investments (Jussawalla 1999 and Umeh 2008), educational and health services (Scott et al. 2004), electricity use (Malmodin et al, 2010), producer and consumer welfare (Torero, Chowdhury and Galdo 2002; Aker and Mbiti 2010; Jensen 2010), extension services (Mittal, Ghandhi and Tripathi 2010), technological adoption and market information systems (Aker 2011; Sekabira et al. 2012; Hoddinott et al. 2013), socio-political enrollment (Castells et al. 2004), prices and markets (Jensen 2007; Aker 2010), agricultural aiding skills like literacy and academic performance (Aker, Ksoll and Lybbert 2012), and sustainable poverty reduction (Bhavnani et al. 2008). However, none of these studies explored the effect of mobile phones or mobile money on household welfare through studying the technologies' influence on gender equality, nutrition, agricultural marketing, and off-farm incomes.

Mobile money services can help make money exchanges quick, prompt and with reduced transactions costs. Money exchange thus business transactions to be financed, hence making buying of farm inputs, selling of farm outputs, and trading of business commodities possible thus enabling agricultural marketing and off-farm income businesses. Mobile money services also allow households to receive remittances from distant relatives and friends. From such remittances, and off-farm income businesses, households can enhance their sources of household off-farm incomes. 


\subsection{Problem Statement}

Aker and Mbiti (2010) identify mobile money as one of the greatest recent significant innovations of mobile phones. Aker and Mbiti, (2010); Nakasone et al. (2014) affirm that empirical research on mobile phones and mobile money is still wanting. Burgess, Pande and Wong (2005) and Levine (2005), argue that extending financial services to the rural poor can bear important effects on economic development and poverty reduction.

However even though, mobile money services render a smooth pathway for money transfers to the unbanked, mobile money services are still poorly regulated. Therefore, numerous questions still need to be empirically answered to guide policy on the welfare impacts of mobile money services (Aker and Mbiti 2010). The impact of mobile money services can well be understood by separating the impact of using a mobile phone from that of using mobile money (Aker 2011). A number of studies however, have focused on studying the impact of mobile phones in general on household welfare (Jensen 2010; Muto and Yamato 2009; Aker 2010 and Muto 2012) but very little attention has been independently accorded to mobile phones and mobile money services. As our contribution in this dissertation, we study welfare impacts of both mobile phones and mobile money services on the same rural households in Uganda.

Kenya's M-pesa (mobile money) is the most documented mobile money service available in Sub

- Sahara Africa (SSA) (Kirui et al. 2013 and Kikulwe et al. 2014); yet country-specific differences exist. Recently, Murendo and Wollni (2016) and Munyegera and Matsumoto (2016) have also documented impacts of mobile money services on remittances and food security in Uganda. However, so much is still desired with regards to the UN's SGDs focus areas of 
significant importance, for instance gender equality, and nutrition that are directly impacted by mobile phones and mobile money services.

Due to the poor infrastructure, Uganda is one of the developing countries with limited number of financial institutions reaching out to the rural poor. Therefore, uses of mobile phones and mobile money services to connect the rural communities to information and markets are of particular importance. Moreover mobile money accounts in Uganda outweigh bank accounts (World Bank 2014). The country has had a steadily increasing number of mobile phone service providers rolling out mobile money services to both rural and urban poor.

By June 2012, mobile money subscribers had, since 2011, increased by $203 \%$ to 5.7 million, transactions number increased by $108 \%$ to 94.5 million, and transactions value increased by $131 \%$ to 4.9 trillion UGX (1.99 US\$ billions), equaling 10\% of Uganda's annual GDP (UBOS 2013). Proportions of mobile money subscriptions based on mobile phone subscription had also greatly risen from $36.5 \%$ in June 2012 to $72.7 \%$ in June 2013. The subscribers' mobile money balances continuously increased since 2011 to 124.4 billion UGX (48 US\$ million) by June 2013 (UCC 2013). Therefore, Uganda provides an interesting research opportunity for this dissertation to contribute to the empirical economic literature.

This dissertation contributes to the existing body of literature by identifying empirically welfare impacts of mobile phones and mobile money on rural smallholder farm households' gender equality through women empowerment, nutrition through dietary quality indicators, household incomes through farm output and off-farm income activities, and agricultural marketing through access and participation in high-value agricultural markets, using the case study of Uganda. 


\subsection{Research Strategy and Objectives}

This dissertation is composed of two papers that are both using panel data collected from rural smallholder farm households in Uganda in 2012 and 2015. The 2012 sample consists of 419 households, and the 2015 sample consists of 455 households. Structured questionnaires were used to collect data through personal interviews with the household head and for some sections also the spouse. The interviews were conducted by trained enumerators who were graduate students or recent university graduates. Data were collected in two districts of central Uganda, namely Luwero and Masaka. We use panel regression models for the empirical econometric analysis.

In an effort to systematically address the identified research gaps, this dissertation focuses on investigating the effect of mobile phones and mobile money use on the welfare of rural farming households in Uganda. Specifically, we analyze:

a) Use patterns of mobile phones and mobile money services among smallholder farming households and determinants of adoption of these technologies.

b) The impact of mobile phone technologies on rural household incomes, gender equality or women empowerment, and nutrition.

c) Impact pathways and effects of gender-disaggregated mobile phone use on farm household nutrition.

d) Impact of mobile money services on farm household welfare (household income and per capita consumption), remittances, and off-farm income.

e) Impact of mobile money services on agricultural marketing and participation in high-value agricultural markets. 
In the first paper, we address objectives (b) and (c) and part of (a), principally using mobile phone use by the households as the treatment variable. We hypothesize that mobile phone use has positive welfare effects for household income, women empowerment, and nutrition. We also hypothesize that female use of mobile phones has larger welfare effects compared to male use. We also hypothesize that income and women empowerment are the key impact pathways through which female mobile phone use positively influences nutrition. We use panel data and panel regression models to study objectives (b) and (a), while simultaneous equations are used for objective (c).

In the second paper, we address objectives (d) and (e), and part of (a), principally using mobile money services use by households as the treatment variable. We hypothesize that use of mobile money services has a positive impact on household incomes, remittances received, and off-farm income. We also hypothesize that use of mobile money services increases households' participation in high-value markets and enhance returns from such markets. Panel regression models are used to test these hypotheses. 


\subsection{Dissertation Outline}

The rest of this dissertation is structured as follows: Chapter 2 presents the first paper, which analyses the impacts of mobile phone use on rural households' income, gender equality, and nutrition. Chapter 3 presents the second paper, analyzing impacts of mobile money use on rural households' income and agricultural marketing. In Chapter 4, we present the dissertations' general conclusions and policy suggestions. The questionnaire that we used for the 2015 survey round is shown in the appendix. Specific limitations to each case study and other specific details are embedded in each respective chapter. 


\section{Can Mobile Phones Improve Gender Equality And Nutrition?}

\section{Panel Data Evidence from Farm Households in Uganda ${ }^{1}$}

Abstract: During the last 10-15 years, mobile phone technologies have been widely adopted in most developing countries, including adoption by rural households that never had access to landline phones before. Existing research shows that use of mobile phones has improved market access for smallholder farmers and thus household income. Beyond income, mobile phones can possibly also affect other dimensions of social welfare, such as gender equality and nutrition. Such broader social welfare effects have hardly been analyzed up till now. Here, we address this research gap, using panel data from smallholder farm households in Uganda. Regression results show that mobile phones have significantly contributed to household income gains and women empowerment. Mobile phone use has also improved household food security and dietary quality. Simultaneous equation models are estimated to show that the positive nutrition effects are primarily channeled through the influence of mobile phones on household income and gender equality. Gender disaggregation reveals that female mobile phone use has stronger positive welfare effects than if males alone use mobile phones. We conclude that equal access to mobile phones cannot only foster economic development, but can also contribute to gender equality, food security, and broader social development.

Key words: mobile phones, women empowerment, dietary diversity, Uganda, gender, incomes

\footnotetext{
${ }^{1}$ This paper has been co-authored with Matin Qaim. I conceptualized the idea, reviewed literature, collected data and analyzed it, and composed it into a paper draft. It's on the paper draft that Matin Qaim provided comments to improve the idea, and as well contributed in the final writing of the paper. The paper has been submitted to the journal "Food Policy" and is under revision.
} 


\subsection{Introduction}

During the last 10-15 years, mobile phone technologies have been widely adopted in developing countries. Mobile phones have significantly improved people's access to information, especially for the rural poor who were never connected to landline phones before. Mobile phones have also reduced other types of transaction costs, thus improving the functioning of markets in various sectors, including agriculture, health, education, financial services, and many more (Fozdar and Kumar, 2007; Jensen, 2007; Duncombe and Boateng, 2009; Aker and Mbiti, 2010; Aker, 2011; Boulos et al., 2011; Aker and Ksoll, 2016; Blauw and Franses, 2016, Nakasone and Torero, 2016). Currently, about 4 billion people globally are using mobile phones. More than two-thirds of these people live in developing countries; with $89 \%$ the highest penetration of mobile phones being recorded in sub-Saharan Africa (PRC, 2015). People in Africa use their mobile phones for a large number of activities and services, including communication with business partners and friends via calls and text messages, access to news and various other types of information, financial transactions, and entertainment (PRC, 2015; UCC, 2015).

A growing body of literature has used micro-level data to analyze the effects of mobile phone use on market access, input and output prices, agricultural production patterns, and household income (Donner, 2007; Jensen, 2007; Aker, 2010; Aker and Mbiti, 2010; Aker, 2011; Kikulwe et al., 2014; Aker and Ksoll, 2016; Nakasone and Torero, 2016; Sekabira and Qaim, 2017). However, mobile phones can possibly also affect various other dimensions of social welfare, such as gender equality and nutrition. Understanding such broader effects is important especially against the background of the United Nations' Sustainable Development Goals, which go far beyond a narrow set of economic development indicators. While a few recent studies have conceptually discussed how mobile phones could influence food security and other welfare dimensions (e.g., 
Aker and Mbiti, 2010; Nakasone et al., 2014; Nakasone and Torero, 2016), empirical data that actually measure such broader social outcomes are scarce.

Here, we address this research gap by using panel data from a farm household survey carried out in Uganda. In particular, beyond looking at income effects, we analyze impacts of mobile phone use on gender equality and nutrition. As in other African countries, mobile phones were adopted very rapidly in Uganda during the last 10 years and are now widely used even by very poor households in remote rural locations (Muto and Yamano, 2009; UCC, 2015; Munyegera and Matsumoto, 2016).

How can mobile phone use influence gender equality and nutrition? A few early studies discussed possible effects on gender roles (Bayes, 2001; Nath, 2001), yet without really evaluating them empirically. For farming households, improved market access through mobile phones will likely increase the degree of commercialization, which could reduce the decisionmaking power of women. Agricultural commercialization is often associated with men taking stronger control of agricultural production and income (Udry, 1996; Fischer and Qaim, 2012). On the other hand, women are often particularly constrained in their access to markets and information. Hence, if women themselves were able to use mobile phones, they could possibly benefit even more than men (Aker and Ksoll, 2016). This could contribute to women empowerment and improved gender equality within the household. Some of our data in Uganda were collected in gender-disaggregated form, so we are able to examine such aspects.

Nutrition effects of mobile phone use can occur through various pathways. Better market access and related income gains are typically associated with improved food security and dietary quality (Sibhatu et al., 2015). Changing gender roles within the household can also influence nutrition 
(Fischer and Qaim, 2012). As women tend to spend more on healthcare and dietary quality than men, women empowerment can improve nutrition even in the absence of income gains (Quisumbing and Maluccio, 2003; Hoddinott, 2012). Furthermore, easier access to all sorts of news services and information through mobile phones may raise people's nutrition knowledge and awareness, which could also contribute to improved dietary practices.

\subsection{Materials and Methods}

\subsubsection{Farm Household Survey}

We use panel data collected in two survey rounds from randomly selected farm households in Masaka and Luwero Districts, Central Uganda. Farmers in these districts grow coffee as their major cash crop, in addition to banana, maize, sweet potato and various other food crops. Within the two districts, we used a two-stage sampling procedure, first selecting three locations and then randomly selecting farmers in each of these locations. The first survey round was conducted in 2012 and covered 419 farm households (Chiputwa et al., 2015). The second survey round was conducted in 2015, targeting the same households. Due to sample attrition, we had to replace 25 households. In addition, we increased the sample size to a total of 455 households in 2015. Additional households and replacements were randomly selected in the same locations. For the analysis, we use the unbalanced panel with 874 observations from 480 households.

In both survey rounds, we used a structured questionnaire for face-to-face interviews with the household head. Certain sections of the questionnaire were also answered separately by the spouse of the household head. The questionnaire focused on agricultural production and marketing, non-farm economic activities and income sources, household consumption, as well as other socio-demographic and contextual details. Household diets were assessed through a 7-day 
food consumption recall covering more than 100 different food items. We also asked for mobile phone ownership and use at the household level, as well as separately for different household members. In this study, we are particularly interested in the mobile phone use by male and female adults in each household. Similarly, ownership of assets was captured in a gender-disaggregated way.

\subsubsection{Measurement of Key Variables}

The main explanatory variable of interest in our analysis is mobile phone (MP) use. We consider a household to be a MP user if at least one adult household member owned a mobile phone during a particular survey year. Furthermore, we differentiate between households where only male adults own a mobile phone (MMP) and households where at least one female adult owns a mobile phone (FMP).

In terms of outcomes, we are particularly interested in household income, gender equality within households, and nutrition. Household income is measured as the total income of the household from all sources over a period of 12 months. For farm income, this also includes the value of production not sold in the market. The cost of production was subtracted for all income derived from self-employed activities. Annual household income is expressed in Ugandan shillings (UGX) (1 US\$ = 2,690 UGX). To be able to compare incomes between the two survey rounds, income in 2012 was adjusted to 2015 values using the official consumer price index (UBOS, 2015).

Gender equality within the household is measured in terms of the proportion of productive assets owned by women or jointly by male and female household members. The proportion refers to the monetary value of the assets. Looking at asset ownership is common in the literature when 
assessing the economic situation of women within households (Quisumbing and Maluccio, 2003; Alsop et al. 2006; Doss et al., 2014). We are interested in how mobile phone use may influence asset ownership. In order to reduce possible issues of reverse causality, we do not consider very durable assets such as land or buildings. We only include short- and medium-term productive assets such as agricultural equipment (hoes, saws, wheelbarrow, sprayers etc.) and vehicles (bike, motorbikes, trucks etc.). In male-dominated households, such assets are predominantly owned by the male household head or other male members. A larger proportion of such assets being owned by females or jointly owned by male and female household members can be interpreted as a higher degree of women empowerment.

Nutrition outcomes can be measured in different ways, including anthropometric indicators, food consumption based measures, and households' subjective assessments of food access (Ruel, 2003; Masset et al., 2012; Shiferaw et al. 2014; Kabunga et al., 2014; Chiputwa and Qaim, 2016). Here, we are particularly interested in how mobile phones affect household food consumption and dietary practices, which we measure through household dietary diversity scores. Dietary diversity scores count the number of different food groups consumed over a specified period of time and are a common tool to assess food security and dietary quality (Ruel, 2003; Jones et al., 2014; Koppmair et al., 2017). Dietary diversity was also shown to be a good proxy of child nutritional status in many situations (Arimond, 2004).

We use the data from the 7-day food consumption recall to calculate two types of dietary diversity scores (DDS) at the household level. First, we use a DDS with 12 food groups, as is common in the literature to calculate household dietary diversity scores for food security assessment (Kennedy et al., 2011). The 12 food groups considered are: cereals; white roots and tubers; vegetables; fruits; meat and poultry; eggs; fish; pulses, legumes and nuts; milk and milk 
products; oils and fats; sugar and honey; and spices, condiments, and beverages. Second, we use a DDS with only 9 food groups, excluding the following three: oils and fats; sugar and honey; and spices, condiments, and beverages. These three food groups are calorie-dense but contribute little to micronutrient consumption. Hence, the DDS with only 9 food groups included is generally considered a better indicator of dietary quality (Sibhatu et al., 2015).

\subsubsection{Econometric Strategy}

We aim to estimate the impact of mobile phone use on household income, gender equality, and nutrition, using the two-round panel data from farm households in Uganda. We start by looking at the three outcomes separately and estimate the following reduced-form panel data models:

$$
\begin{gathered}
Y_{i t}=\beta_{0}+\beta_{1} M P_{i t}+\beta_{2}^{\prime} \mathbf{X}_{i t}+\beta_{3} T_{t}+\varepsilon_{i t} \\
G E_{i t}=\beta_{0}+\beta_{1} M P_{i t}+\beta_{2}^{\prime} \mathbf{X}_{i t}+\beta_{3} T_{t}+\varepsilon_{i t} \\
N_{i t}=\beta_{0}+\beta_{1} M P_{i t}+\beta_{2}^{\prime} \mathbf{X}_{i t}+\beta_{3} T_{t}+\varepsilon_{i t}
\end{gathered}
$$

where $Y_{i t}, G E_{i t}$, and $N_{i t}$ are the indicators of income, gender equality, and nutrition, as explained above, referring to household $i$ in year $t . M P_{i t}$ is a dummy variable that takes a value of one if any adult in the household owned and used a mobile phone in year $t$, and zero otherwise. $\mathbf{X}_{i t}$ is a vector of farm, household, and contextual characteristics, $T_{t}$ is a year dummy for 2015 , and $\varepsilon_{i t}$ is a normally distributed random error term. Equations (2.1) to (2.3) are estimated separately. We are particularly interested in the estimates for $\beta_{1}$. Positive and significant estimates would imply that mobile phone use increases household income, gender equality, and nutrition after controlling for other factors. 
In a second set of estimates, we use the same reduced-form equations (2.1) to (2.3) but replace the aggregate mobile phone use dummy with gender-disaggregated dummies. As explained above, we use $F M P_{i t}$ for households where at least one female adult owns a mobile phone and $M M P_{i t}$ for households where only male adults own and use mobile phones. To avoid collinearity problems, we estimate separate models with each gender-specific mobile phone dummy.

All models are estimated with random effects (RE) panel estimator. However, mobile phone use is not a completely random variable. In our sample, households deliberately chose whether or not to adopt mobile phone technology based on preferences and constraints, some of which may be unobserved. If mobile phone use is correlated with unobserved factors that also influence the outcome variables directly, the RE estimator can lead to biased estimates of $\beta_{1}$. To test for unobserved heterogeneity and reduce potential bias, we also use a fixed effects (FE) estimator, which employs differencing techniques within households over time and therefore eliminates any bias from time-invariant unobserved heterogeneity. RE and FE estimates are compared with a Hausman test (Cameron and Trivedi, 2005). An insignificant Hausman test statistic suggests that the RE estimator leads to consistent results and is preferable due to its higher efficiency. A significant test statistic, on the other hand, points at problems with unobserved heterogeneity, so that the FE estimator is preferred.

One drawback of the FE estimator is that for each variable of interest it requires sufficient variation within households over time to produce efficient estimates. Completely time-invariant variables drop out during estimation, and for variables with little time variation the estimates are often unreliable. One alternative is the Mundlak approach that produces more efficient estimates for variables with little time variation (Mundlak, 1978). The Mundlak approach builds on the FE 
estimator but adds variable group means to reduce issues of unobserved heterogeneity. As the time variation in our mobile phone dummies is limited, we use the Mundlak estimator in addition to the standard RE and FE models.

\subsubsection{Modelling Impact Pathways}

As discussed above, the impact of mobile phone use on household nutrition will likely be channeled through the effects of mobile phones on income and gender equality. To model these causal pathways more explicitly, we develop and estimate a system of simultaneous equations as follows:

$$
\begin{gathered}
N_{i t}=\alpha_{0}+\alpha_{1} Y_{i t}+\alpha_{2} G E_{i t}+\alpha_{3}^{\prime} \mathbf{X}_{i t}+\alpha_{4} T_{t}+\mu_{i t 1} \\
Y_{i t}=\beta_{0}+\beta_{1} M P_{i t}+\beta_{2}^{\prime} \mathbf{Z}_{i t}+\beta_{3} T_{t}+\mu_{i t 2} \\
G E_{i t}=\gamma_{0}+\gamma_{1} M P_{i t}+\gamma_{3}^{\prime} \mathbf{L}_{i t}+\gamma_{4} T_{t}+\mu_{i t 3} \\
M P_{i t}=\theta_{0}+\theta_{1}^{\prime} \mathbf{M}_{i t}+\theta_{2} T_{t}+\mu_{i t 4}
\end{gathered}
$$

In equation (2.4), nutrition $\left(N_{i t}\right)$ is modeled as a function of household income $\left(Y_{i t}\right)$, gender equality $\left(G E_{i t}\right)$, and other socioeconomic factors $\left(\mathbf{X}_{i t}\right)$. In equations (2.5) and (2.6), income and gender equality are modeled as functions of mobile phone use $\left(M P_{i t}\right)$ and other socioeconomic characteristics ( $\mathbf{Z}_{i t}$ and $\mathbf{L}_{i t}$ ). In equation (2.7), mobile phone use is itself considered endogenous and explained by a vector of socioeconomic variables $\left(\mathbf{M}_{i t}\right)$. Equations (2.4) to (2.7) are estimated simultaneously using three-stage least squares (Zellner and Theil, 1962).

The vectors $\mathbf{X}_{i t}, \mathbf{Z}_{i t}, \mathbf{L}_{i t}$, and $\mathbf{M}_{i t}$ include farm, household, and contextual characteristics that may overlap across the different equations. For instance, in all equations we include age, 
education, and gender of the household head, household size (measured in terms of adult equivalents), land owned, distance to road, and a district dummy that can all influence mobile phone adoption and the different welfare outcomes. For $\mathbf{M}_{i t}$ in equation (2.7) we additionally include two instruments that are correlated with mobile phone adoption but have no effect on household welfare through other pathways. These instruments are the strengths of the mobile network coverage in the location of household $i$ and the number of households using mobile phones out of the 10 closest neighbors. Valid instruments control for unobserved heterogeneity and also for possible reverse causality. For instance, it could be possible that the links between mobile phone use, household income, and gender equality work in several directions. Since the FE and Mundlak estimators cannot control for reverse causality, estimates from this simultaneous equation model with instruments for mobile phone use can also serve as a robustness check for the reduced-form results from equations (2.1) to (2.3).

\subsection{Results and Discussion}

\subsubsection{Descriptive Statistics}

Table 1 shows the patterns of mobile phone use by households in our sample and how these patterns developed between the two survey rounds. In 2012, $76 \%$ of the households used mobile phones. By 2015, this proportion had increased to $89 \%$. Table 2.1 also shows who within the households actually used mobile phones. While the number of male mobile phone users did not change much, the number of households in which females also use mobile phones increased substantially between 2012 and 2015 . 
Table 2.1: Number of households in the sample using and not using mobile phones

\begin{tabular}{lcccccc}
\hline & \multicolumn{2}{c}{2012} & \multicolumn{2}{c}{2015} & \multicolumn{2}{c}{ Pooled sample } \\
\cline { 2 - 6 } & Non-users & Users & Non-users & Users & Non-users & Users \\
\hline Mobile phone use (MP) & 99 & 320 & 49 & 406 & 148 & 726 \\
& $(23.63)$ & $(76.37)$ & $(10.77)$ & $(89.23)$ & $(16.93)$ & $(83.07)$ \\
Mobile phone used by & 217 & 202 & 160 & 295 & 377 & 497 \\
female adults (FMP) & $(51.79)$ & $(48.21)$ & $(35.16)$ & $(64.84)$ & $(43.14)$ & $(56.86)$ \\
Mobile phone used only & 314 & 105 & 343 & 112 & 657 & 217 \\
by male adults (MMP) & $(74.94)$ & $(25.06)$ & $(75.38)$ & $(24.62)$ & $(75.17)$ & $(24.83)$ \\
\hline
\end{tabular}

Note: Percentage shares are shown in parentheses.

Table 2.2 shows descriptive statistics for the socioeconomic characteristics that we use as explanatory variables in the econometric models, differentiating between mobile phone users and non-users. Some significant differences can be observed. Mobile phone users have larger farms, more family members, as well as younger and better educated household heads than non-users. Tables, 2.9 and 2.10 in the appendix 2 of supplementary material show the same variables, differentiating between female and male only mobile phone users.

Table 2.2: Socioeconomic characteristics by mobile phone use

\begin{tabular}{lcccccc}
\hline & \multicolumn{2}{c}{2012} & \multicolumn{2}{c}{2015} & \multicolumn{2}{c}{ Pooled sample } \\
\cline { 2 - 7 } & $\begin{array}{c}\text { Non-users } \\
(\mathrm{N}=99)\end{array}$ & $\begin{array}{c}\text { Users } \\
(\mathrm{N}=320)\end{array}$ & $\begin{array}{c}\text { Non-users } \\
(\mathrm{N}=49)\end{array}$ & $\begin{array}{c}\text { Users } \\
(\mathrm{N}=406)\end{array}$ & $\begin{array}{c}\text { Non-users } \\
(\mathrm{N}=148)\end{array}$ & $\begin{array}{c}\text { Users } \\
(\mathrm{N}=726)\end{array}$ \\
\hline Age of household head (years) & 58.051 & $50.897^{* * * *}$ & 62.551 & $53.867^{* * *}$ & 59.541 & $52.558^{* * *}$ \\
& $(16.776)$ & $(13.031)$ & $(13.637)$ & $(13.556)$ & $(15.902)$ & $(13.399)$ \\
Education of household head & 4.727 & $7.141^{* * *}$ & 4.939 & $7.003^{* * *}$ & 4.797 & $7.063^{* * * *}$ \\
(years) & $(3.454)$ & $(3.490)$ & $(3.369)$ & $(3.646)$ & $(3.416)$ & $(3.577)$ \\
Male household head (dummy) & 0.646 & $0.791^{* * *}$ & 0.592 & $0.800^{* * *}$ & 0.628 & $0.796^{* * * *}$ \\
Migrant household (dummy) & 0.273 & 0.200 & 0.061 & $0.158^{* *}$ & 0.203 & 0.176 \\
Household size (AE) & 4.176 & $5.489^{* * *}$ & 3.436 & $5.369^{* * *}$ & 3.931 & $5.422^{* * * *}$ \\
& $(2.197)$ & $(2.869)$ & $(2.323)$ & $(2.539)$ & $(2.258)$ & $(2.688)$ \\
Land owned (ha) & 1.827 & $2.415^{* * *}$ & 1.759 & 2.423 & 1.804 & $2.419^{* * *}$ \\
& $(1.216)$ & $(1.867)$ & $(1.039)$ & $(3.218)$ & $(1.157)$ & $(2.705)$ \\
Distance to tarmac road (km) & 15.697 & 16.537 & 16.235 & 15.346 & 15.875 & 15.871 \\
& $(11.103)$ & $(11.575)$ & $(10.724)$ & $(10.062)$ & $(10.946)$ & $(10.763)$ \\
Residence in Masaka & 0.242 & $0.575^{* * *}$ & 0.245 & $0.483^{* * *}$ & 0.243 & $0.523^{* * * *}$ \\
Neighbors using mobile phone & 3.581 & $6.156^{* * *}$ & 1.541 & $9.145^{* * *}$ & 2.905 & $7.828^{* * *}$ \\
& $(3.395)$ & $(3.379)$ & $(1.903)$ & $(1.871)$ & $(3.130)$ & $(3.031)$ \\
Network coverage & 0.384 & $0.806^{* * *}$ & 0.041 & $0.958^{* * *}$ & 0.270 & $0.891^{* * *}$ \\
\hline
\end{tabular}

Notes: Mean values are shown with standard deviations in parentheses. AE, adult equivalents. Differences in means between users and non-users are tested for statistical significance. ${ }^{* * *} \mathrm{p}<0.01,{ }^{* *} \mathrm{p}<0.05,{ }^{*} \mathrm{p}<0.1$ 
Table 2.3 shows descriptive statistics for the outcome variables of interest in this study. Households using mobile phones have higher incomes and higher levels of gender equality than households without mobile phones. In households without mobile phones, women own less than $50 \%$ of the productive assets alone or together with male household members. In mobile phoneusing households, $63 \%$ of the assets are owned by women or jointly by male and female household members. Dietary diversity is also higher in households with mobile phones. Differences are statistically significant for both dietary diversity scores, with 12 and 9 food groups.

Table 2.3: Household income, gender equality, and nutrition by mobile phone use

\begin{tabular}{|c|c|c|c|c|c|c|}
\hline & \multicolumn{2}{|c|}{2012} & \multicolumn{2}{|c|}{2015} & \multicolumn{2}{|c|}{ Pooled sample } \\
\hline & Non-users & Users & Non-users & Users & Non-users & Users \\
\hline \multicolumn{7}{|l|}{ Mobile phone use (MP) } \\
\hline \multirow[t]{2}{*}{ Income (million UGX) } & 1.482 & $3.576^{* * *}$ & 1.972 & $3.549^{* * *}$ & 1.644 & $3.561^{* * *}$ \\
\hline & $(1.979)$ & $(3.505)$ & $(3.016)$ & $(3.481)$ & $(2.374)$ & $(3.489)$ \\
\hline $\begin{array}{l}\text { Proportion of assets owned } \\
\text { by women or jointly }\end{array}$ & 0.466 & $0.549^{* *}$ & 0.489 & $0.689^{* * *}$ & 0.474 & $0.627^{* * *}$ \\
\hline \multirow[t]{2}{*}{ DDS 12 food groups } & 8.808 & $9.544^{* * *}$ & 8.489 & $9.246^{* * *}$ & 8.703 & $9.377^{* * *}$ \\
\hline & $(1.979)$ & $(1.150)$ & $(1.488)$ & $(1.431)$ & $(1.831)$ & $(1.473)$ \\
\hline \multirow[t]{2}{*}{ DDS 9 food groups } & 6.444 & $6.981^{* * * *}$ & 6.000 & $6.534^{* * * *}$ & 6.297 & $6.731^{* * *}$ \\
\hline & $(0.161)$ & $(0.074)$ & $(0.152)$ & $(0.061)$ & $(0.119)$ & $(0.048)$ \\
\hline \multicolumn{7}{|l|}{ Female use (FMP) } \\
\hline \multirow[t]{2}{*}{ Income (million UGX) } & 2.636 & $3.559^{* * *}$ & 2.655 & $3.773^{* * *}$ & 2.644 & $3.686^{* * *}$ \\
\hline & $(2.888)$ & $(3.695)$ & $(2.878)$ & $(3.692)$ & $(2.879)$ & $(3.691)$ \\
\hline $\begin{array}{l}\text { Proportion of assets owned } \\
\text { by women or jointly }\end{array}$ & 0.498 & $0.563^{* *}$ & 0.612 & $0.698^{* * * *}$ & 0.546 & $0.643^{* * *}$ \\
\hline \multirow[t]{2}{*}{ DDS 12 food groups } & 9.147 & $9.609^{* * *}$ & 8.831 & $9.356^{* * *}$ & 9.013 & $9.453^{* * *}$ \\
\hline & $(1.707)$ & $(1.577)$ & $(1.579)$ & $(1.351)$ & $(1.659)$ & $(1.452)$ \\
\hline \multirow[t]{2}{*}{ DDS 9 food groups } & 6.673 & $7.049^{* * *}$ & 6.219 & $6.617^{* * *}$ & 6.480 & $6.793^{* * *}$ \\
\hline & $(1.449)$ & $(1.345)$ & $(1.302)$ & $(1.157)$ & $(1.405)$ & $(1.254)$ \\
\hline \multicolumn{7}{|l|}{ Male use (MMP) } \\
\hline \multirow[t]{2}{*}{ Income (million UGX) } & 3.097 & 3.034 & 3.493 & 3.033 & 3.304 & 3.033 \\
\hline & $(3.511)$ & $(2.730)$ & $(3.636)$ & $(2.869)$ & $(3.579)$ & $(2.796)$ \\
\hline $\begin{array}{l}\text { Proportion of assets owned } \\
\text { by women or jointly }\end{array}$ & 0.532 & 0.519 & 0.668 & 0.667 & 0.603 & 0.596 \\
\hline \multirow[t]{2}{*}{ DDS 12 food groups } & 9.363 & 9.390 & 9.227 & 8.973 & 9.292 & 9.175 \\
\hline & $(1.662)$ & $(1.661)$ & $(1.402)$ & $(1.597)$ & $(1.532)$ & $(1.638)$ \\
\hline \multirow[t]{2}{*}{ DDS 9 food groups } & 6.847 & 6.876 & 6.531 & 6.313 & 6.682 & 6.585 \\
\hline & $(1.419)$ & $(1.392)$ & $(1.167)$ & $(1.376)$ & $(1.302)$ & $(1.409)$ \\
\hline
\end{tabular}

Notes: Mean values are shown with standard deviations in parentheses. DDS, dietary diversity score. Differences in means between users and non-users are tested for statistical significance. ${ }^{* * *} \mathrm{p}<0.01,{ }^{* *} \mathrm{p}<0.05,{ }^{*} \mathrm{p}<0.1$ 
The middle and lower parts of Table 2.3 show the same outcome variables differentiated by female and male mobile phone use. While male-only use of mobile phones is not associated with significant differences in any of the outcome variables, female use of mobile phones is. Households in which females use mobile phones have higher incomes, more equal gender relations, and higher levels of dietary diversity than households in which females do not use mobile phones. The results in Table 2.3 lend support to the hypothesis that mobile phone use by females has stronger positive social welfare effects than mobile phone use by males alone, although these simple comparisons should not be over-interpreted in a causal sense. Causal relationships are analyzed more formally below.

\subsubsection{Aggregate Effects of Mobile Phone Use}

Table 2.4 shows the estimation results of equations (2.1) and (2.2), using random effects (RE), fixed effects (FE), and Mundlak (MK) estimators, as explained above. For the income model, the RE estimator produces a positive and significant coefficient for the mobile phone use dummy. However, the Hausman test is statistically significant, so that the RE estimate may be biased. With the FE estimator, the effect of mobile phones is insignificant, due to a relatively large standard error. But the MK estimator produces a positive and significant coefficient, which we use here for interpretation. The results in column (3) of Table 2.4 suggest that mobile phone use has increased household income by 0.43 million UGX, which is equivalent to a $26 \%$ gain compared to the mean income of households without mobile phones. This is a substantial effect that can be explained through mobile phones improving households' access to information and markets, lower transaction costs, and hence higher returns in agricultural and non-agricultural activities. That mobile phones can have sizeable economic effects at the micro level was also shown in number of previous studies (Donner, 2007; Jensen, 2007; Muto and Yamano, 2009; 
Aker, 2010; Blauw and Franses, 2016).

Table 2.4: Effects of mobile phone use on household income and gender equality

\begin{tabular}{|c|c|c|c|c|c|c|}
\hline & \multicolumn{3}{|c|}{ Income (million UGX) } & \multicolumn{3}{|c|}{ Gender equality (proportion of assets) } \\
\hline & (1) RE & (2) FE & (3) $\mathrm{MK}$ & (4) RE & (5) FE & (6) $\mathrm{MK}$ \\
\hline \multirow[t]{2}{*}{ MP use (dummy) } & $0.561^{* * *}$ & 0.410 & $0.433^{* *}$ & $0.090^{* * *}$ & 0.075 & $0.088^{* * * *}$ \\
\hline & $(0.182)$ & $(0.279)$ & $(0.181)$ & $(0.031)$ & $(0.047)$ & $(0.031)$ \\
\hline \multirow{2}{*}{$\begin{array}{l}\text { Education of head } \\
\text { (years) }\end{array}$} & $0.094^{* * *}$ & 0.020 & -0.013 & 0.005 & -0.005 & -0.004 \\
\hline & $(0.022)$ & $(0.051)$ & $(0.052)$ & $(0.004)$ & $(0.009)$ & $(0.009)$ \\
\hline \multirow[t]{2}{*}{ Male head (dummy) } & $0.704^{* * *}$ & & $1.528^{* * *}$ & $-0.082^{* * *}$ & & -0.067 \\
\hline & $(0.164)$ & & $(0.518)$ & $(0.028)$ & & $(0.089)$ \\
\hline \multirow[t]{2}{*}{ Household size (AE) } & $0.054^{*}$ & -0.061 & -0.072 & $0.021^{* * * *}$ & $0.019^{* *}$ & $0.019^{* *}$ \\
\hline & $(0.028)$ & $(0.053)$ & $(0.052)$ & $(0.005)$ & $(0.009)$ & $(0.009)$ \\
\hline \multirow[t]{2}{*}{ Land owned (ha) } & $0.518^{* * *}$ & & $0.209^{* *}$ & $-0.020^{* *}$ & & -0.021 \\
\hline & $(0.051)$ & & $(0.092)$ & $(0.009)$ & & $(0.016)$ \\
\hline \multirow[t]{2}{*}{ Age of head (years) } & $-0.018^{* * *}$ & -0.005 & -0.019 & -0.0004 & 0.001 & 0.001 \\
\hline & $(0.005)$ & $(0.016)$ & $(0.016)$ & $(0.001)$ & $(0.003)$ & $(0.003)$ \\
\hline \multirow{2}{*}{$\begin{array}{l}\text { Distance to tarmac road } \\
(\mathrm{km})\end{array}$} & -0.007 & & -0.005 & -0.0002 & & -0.002 \\
\hline & $(0.007)$ & & $(0.017)$ & $(0.001)$ & & $(0.003)$ \\
\hline \multirow[t]{2}{*}{ Year 2015 (dummy) } & 0.151 & 0.128 & 0.154 & $0.123^{* * *}$ & $0.125^{* * *}$ & $0.120^{* * *}$ \\
\hline & $(0.116)$ & $(0.130)$ & $(0.122)$ & $(0.019)$ & $(0.022)$ & $(0.021)$ \\
\hline \multirow[t]{2}{*}{ Migrant (dummy) } & 0.107 & 0.164 & 0.220 & $-0.047^{*}$ & -0.026 & -0.031 \\
\hline & $(0.159)$ & $(0.229)$ & $(0.227)$ & $(0.027)$ & $(0.039)$ & $(0.039)$ \\
\hline \multirow[t]{2}{*}{ Masaka (dummy) } & $0.999^{* * *}$ & & $1.016^{* * *}$ & 0.029 & & 0.023 \\
\hline & $(0.145)$ & & $(0.147)$ & $(0.025)$ & & $(0.026)$ \\
\hline \multirow[t]{2}{*}{ Constant } & $7.261^{* * * *}$ & $9.094^{* * *}$ & $7.395^{\text {*** }}$ & $0.418^{* * *}$ & $0.356^{* *}$ & $0.419^{* * *}$ \\
\hline & $(0.404)$ & $(0.996)$ & $(0.443)$ & $(0.069)$ & $(0.169)$ & $(0.077)$ \\
\hline Observations & 874 & 874 & 874 & 874 & 874 & 874 \\
\hline No. of households & 480 & 480 & 480 & 480 & 480 & 480 \\
\hline Wald $\chi 2$ / $F$-value & $354.12^{* * *}$ & 1.06 & $393.48^{* * *}$ & $103.21^{* * * *}$ & $9.44^{* * *}$ & $105.82^{* * *}$ \\
\hline Hausman $\chi 2$ & & $11.77^{*}$ & & & 3.05 & \\
\hline
\end{tabular}

Notes: Coefficient estimates are shown with standard errors in parentheses. RE, random effects estimator; FE, fixed effects estimator; MK, Mundlak estimator; MP, mobile phone; UGX, Ugandan shillings; AE, adult equivalents. $\mathrm{p}<0.01,{ }^{* *} \mathrm{p}<0.05,{ }^{*} \mathrm{p}<0.1$

Columns (4) to (6) of Table 2.4 show that mobile phone use also improves gender equality. As the Hausman test statistic in this model is insignificant, we rely on the RE estimate for interpretation. Mobile phone use increases the proportion of productive assets owned by women or jointly by women and men by 0.09 , which is equivalent to a $19 \%$ increase over the mean female asset ownership in households without mobile phone. Such effects on gender equality were not analyzed before, but they are plausible given that women are often particularly constrained in their access to information and markets and may therefore benefit over- 
proportionally from the use of mobile phone technologies.

Results of the effects of mobile phones on household nutrition are shown in Table 2.5. As explained we use a dietary diversity score (DDS) with 12 food groups to assess household food security and a DDS with 9 food groups to proxy dietary quality. For both outcome variables, the Hausman test is insignificant, so we use the RE estimates for interpretation. Mobile phone use significantly improves access to food and dietary quality. This is consistent with what was hypothesized in the literature (Nakasone and Torero, 2016), even though this hypothesis had not been tested before empirically.

Table 2.5: Effects of mobile phone use on household nutrition

\begin{tabular}{|c|c|c|c|c|c|c|}
\hline & \multicolumn{3}{|c|}{ DDS 12 food groups } & \multicolumn{3}{|c|}{ DDS 9 food groups } \\
\hline & (1) RE & (2) $\mathrm{FE}$ & (3) $\mathrm{MK}$ & (4) RE & (5) FE & (6) $\mathrm{MK}$ \\
\hline \multirow[t]{2}{*}{ MP use (dummy) } & $0.336^{* *}$ & 0.202 & $0.300^{* * *}$ & $0.236^{*}$ & 0.173 & 0.210 \\
\hline & $(0.150)$ & $(0.233)$ & $(0.150)$ & $(0.128)$ & $(0.202)$ & $(0.129)$ \\
\hline \multirow{2}{*}{$\begin{array}{l}\text { Education of household } \\
\text { head (years) }\end{array}$} & $0.059^{* * *}$ & $0.079^{*}$ & 0.042 & $0.043^{* * *}$ & 0.058 & 0.027 \\
\hline & $(0.018)$ & $(0.043)$ & $(0.044)$ & $(0.016)$ & $(0.037)$ & $(0.038)$ \\
\hline \multirow[t]{2}{*}{ Male head (dummy) } & 0.155 & & $1.495^{* * *}$ & 0.144 & & $1.297^{* * *}$ \\
\hline & $(0.135)$ & & $(0.434)$ & $(0.114)$ & & $(0.376)$ \\
\hline \multirow[t]{2}{*}{ Household size (AE) } & $0.116^{* * *}$ & $0.078^{*}$ & 0.068 & $0.082^{* * *}$ & 0.056 & 0.048 \\
\hline & $(0.023)$ & $(0.044)$ & $(0.044)$ & $(0.019)$ & $(0.039)$ & $(0.038)$ \\
\hline \multirow[t]{2}{*}{ Land owned (ha) } & 0.029 & & 0.102 & $0.065^{*}$ & & $0.132^{* *}$ \\
\hline & $(0.042)$ & & $(0.077)$ & $(0.036)$ & & $(0.067)$ \\
\hline \multirow[t]{2}{*}{ Age of head (years) } & $-0.009^{* *}$ & 0.012 & -0.004 & $-0.006^{*}$ & 0.004 & -0.010 \\
\hline & $(0.004)$ & $(0.013)$ & $(0.014)$ & $(0.003)$ & $(0.011)$ & $(0.012)$ \\
\hline \multirow{2}{*}{$\begin{array}{l}\text { Distance to tarmac road } \\
(\mathrm{km})\end{array}$} & 0.002 & & 0.006 & 0.004 & & 0.008 \\
\hline & $(0.006)$ & & $(0.014)$ & $(0.005)$ & & $(0.012)$ \\
\hline \multirow[t]{2}{*}{ Year 2015} & $-0.264^{* * *}$ & $-0.325^{* * *}$ & $-0.270^{* * *}$ & $-0.416^{* * *}$ & $-0.458^{* * *}$ & $-0.404^{* * *}$ \\
\hline & $(0.097)$ & $(0.109)$ & $(0.102)$ & $(0.083)$ & $(0.095)$ & $(0.089)$ \\
\hline \multirow[t]{2}{*}{ Migrant (dummy) } & $-0.252^{*}$ & $-0.478^{* *}$ & $-0.414^{* *}$ & $-0.221^{* *}$ & $-0.410^{* *}$ & $-0.351^{* *}$ \\
\hline & $(0.131)$ & $(0.191)$ & $(0.190)$ & $(0.112)$ & $(0.166)$ & $(0.165)$ \\
\hline \multirow[t]{2}{*}{ Masaka (dummy) } & -0.119 & & -0.088 & -0.123 & & -0.097 \\
\hline & $(0.119)$ & & $(0.121)$ & $(0.101)$ & & $(0.103)$ \\
\hline \multirow[t]{2}{*}{ Constant } & $8.581^{* * *}$ & $7.822^{* * *}$ & $8.730^{* * * *}$ & $6.212^{* * * *}$ & $5.996^{* * *}$ & $6.278^{* * *}$ \\
\hline & $(0.332)$ & $(0.832)$ & $(0.366)$ & $(0.283)$ & $(0.722)$ & $(0.310)$ \\
\hline Observations & 874 & 874 & 874 & 874 & 874 & 874 \\
\hline No. of households & 480 & 480 & 480 & 480 & 480 & 480 \\
\hline Wald $\chi 2$ / $F$-value & $92.73^{* * *}$ & $3.45^{* * *}$ & $111.63^{* * *}$ & $94.49^{* * *}$ & $5.61^{* * *}$ & $110.75^{\text {*** }}$ \\
\hline Hausman $\chi^{2}$ & & 6.56 & & & 3.98 & \\
\hline
\end{tabular}

Notes: Coefficient estimates are shown with standard errors in parentheses. DDS, dietary diversity score; RE, random effects estimator; FE, fixed effects estimator; MK, Mundlak estimator; MP, mobile phone; AE, adult equivalents. ${ }^{* * *} \mathrm{p}<0.01,{ }^{* *} \mathrm{p}<0.05,{ }^{*} \mathrm{p}<0.1$ 
A few other explanatory variables in Table 2.5 also have significant effects. Education of the household head and household size tend to increase dietary diversity at the household level, while age decreases dietary diversity, as one would expect. The 2015 dummy has a negative effect, which can be explained by lower rainfall in the 2014 season and thus poorer agricultural harvests than in the 2011 season that was captured in the 2012 survey round.

\subsubsection{Gender Disaggregated Effects of Mobile Phone use}

So far, we have used household-level mobile phone use as the main explanatory variable in the models, regardless of who in the household actually used mobile phones. We now differentiate between female and male mobile phone use to gain further insights into gendered effects. Table 2.6 shows the estimation results for household income and gender equality. Female mobile phone use has significantly positive effects on both outcome variables, while male mobile phone use has not. The same pattern is also observed for the effects of female and male mobile phone use on household diets, which are shown in Table 2.7.

The insignificant coefficients of male mobile phone use should not be over-interpreted. As explained, the MMP dummy only captures households in which males alone use mobile phones. In many cases, both male and female household members use mobile phones, and these cases are captured by the FMP dummy. Nevertheless, that female mobile phone use seems to be more important for positive social welfare effects than male mobile phone use is remarkable and in line

with our hypothesis on gendered implications. Women benefit over-proportionally from the use of mobile phone technologies, and larger economic benefits are also reflected in enhanced gender equality within the household and better household nutrition. 
Table 2.6: Effects of female and male mobile phone use on household income and gender equality

\begin{tabular}{|c|c|c|c|c|c|c|c|c|c|c|c|c|}
\hline & \multicolumn{6}{|c|}{ Income (million UGX) } & \multicolumn{6}{|c|}{ Gender equality (proportion of assets owned) } \\
\hline & (1) RE & (2) FE & (3) MK & (4) RE & (5) FE & (6) MK & (7) $\mathrm{RE}$ & (8) FE & (9) $\mathrm{MK}$ & (10)RE & $(11) \mathrm{FE}$ & (12)MK \\
\hline FMP use (dummy) & $\begin{array}{l}0.320^{* *} \\
(0.137)\end{array}$ & $\begin{array}{l}-0.259 \\
(0.295)\end{array}$ & $\begin{array}{l}0.241^{*} \\
(0.138)\end{array}$ & & & & $\begin{array}{l}0.047^{* *} \\
(0.024)\end{array}$ & $\begin{array}{c}0.063 \\
(0.050)\end{array}$ & $\begin{array}{l}0.047^{*} \\
(0.024)\end{array}$ & & & \\
\hline $\begin{array}{l}\text { MMP use } \\
\text { (dummy) }\end{array}$ & & & & $\begin{array}{l}-0.227 \\
(0.152)\end{array}$ & $\begin{array}{c}0.091 \\
(0.294)\end{array}$ & $\begin{array}{l}-0.237 \\
(0.151)\end{array}$ & & & & $\begin{array}{c}0.006 \\
(0.026)\end{array}$ & $\begin{array}{l}-0.011 \\
(0.049)\end{array}$ & $\begin{array}{c}0.006 \\
(0.026)\end{array}$ \\
\hline $\begin{array}{l}\text { Education of head } \\
\text { (years) }\end{array}$ & $\begin{array}{l}0.094^{* * * *} \\
(0.022)\end{array}$ & $\begin{array}{c}0.024 \\
(0.051)\end{array}$ & $\begin{array}{l}-0.009 \\
(0.052)\end{array}$ & $\begin{array}{l}0.099^{* * * *} \\
(0.022)\end{array}$ & $\begin{array}{c}0.024 \\
(0.051)\end{array}$ & $\begin{array}{l}-0.009 \\
(0.052)\end{array}$ & $\begin{array}{c}0.005 \\
(0.004)\end{array}$ & $\begin{array}{c}-0.004 \\
(0.009)\end{array}$ & $\begin{array}{l}-0.003 \\
(0.009)\end{array}$ & $\begin{array}{l}0.006 * \\
(0.004)\end{array}$ & $\begin{array}{l}-0.004 \\
(0.009)\end{array}$ & $\begin{array}{l}-0.003 \\
(0.009)\end{array}$ \\
\hline $\begin{array}{l}\text { Male head } \\
\text { (dummy) }\end{array}$ & $\begin{array}{l}0.788^{* * * *} \\
(0.168)\end{array}$ & & $\begin{array}{l}1.623^{* * * *} \\
(0.517)\end{array}$ & $\begin{array}{l}0.773^{* * * *} \\
(0.170)\end{array}$ & & $\begin{array}{l}1.647^{* * * *} \\
(0.518)\end{array}$ & $\begin{array}{l}-0.069^{* *} \\
(0.028)\end{array}$ & & $\begin{array}{l}-0.047 \\
(0.089)\end{array}$ & $\begin{array}{c}-0.082^{* * * *} \\
(0.029)\end{array}$ & & $\begin{array}{l}-0.047 \\
(0.089)\end{array}$ \\
\hline Household size & $0.061^{* * *}$ & -0.057 & -0.066 & $0.069^{* * *}$ & -0.054 & -0.070 & $0.022^{* * * *}$ & $0.021^{* *}$ & $0.021^{* * *}$ & $0.024^{* * * *}$ & $0.020^{* * *}$ & $0.021^{* * *}$ \\
\hline$(\mathrm{AE})$ & $(0.028)$ & $(0.053)$ & $(0.052)$ & $(0.027)$ & $(0.053)$ & $(0.052)$ & $(0.005)$ & $(0.009)$ & $(0.009)$ & $(0.005)$ & $(0.009)$ & $(0.009)$ \\
\hline Land owned (ha ) & $\begin{array}{l}0.529^{* * * *} \\
(0.051)\end{array}$ & & $\begin{array}{l}0.204^{* * *} \\
(0.092)\end{array}$ & $\begin{array}{l}0.533^{* * * *} \\
(0.051)\end{array}$ & & $\begin{array}{l}0.201^{* * *} \\
(0.092)\end{array}$ & $\begin{array}{l}-0.018^{* * *} \\
(0.009)\end{array}$ & & $\begin{array}{l}-0.022 \\
(0.016)\end{array}$ & $\begin{array}{l}-0.018^{* * *} \\
(0.009)\end{array}$ & & $\begin{array}{l}-0.022 \\
(0.016)\end{array}$ \\
\hline $\begin{array}{l}\text { Age of head } \\
\text { (years) }\end{array}$ & $\begin{array}{c}-0.020^{* * * *} \\
(0.005)\end{array}$ & $\begin{array}{l}-0.007 \\
(0.016)\end{array}$ & $\begin{array}{l}-0.021 \\
(0.016)\end{array}$ & $\begin{array}{l}-0.021^{* * * *} \\
(0.005)\end{array}$ & $\begin{array}{l}-0.006 \\
(0.016)\end{array}$ & $\begin{array}{l}-0.023 \\
(0.016)\end{array}$ & $\begin{array}{l}-0.001 \\
(0.001)\end{array}$ & $\begin{array}{c}0.001 \\
(0.003)\end{array}$ & $\begin{array}{c}0.001 \\
(0.003)\end{array}$ & $\begin{array}{l}-0.001 \\
(0.001)\end{array}$ & $\begin{array}{c}0.000 \\
(0.003)\end{array}$ & $\begin{array}{c}0.001 \\
(0.003)\end{array}$ \\
\hline $\begin{array}{l}\text { Distance to tarmac } \\
\text { road }(\mathrm{km})\end{array}$ & $\begin{array}{l}-0.007 \\
(0.007)\end{array}$ & & $\begin{array}{l}-0.008 \\
(0.017)\end{array}$ & $\begin{array}{l}-0.008 \\
(0.007)\end{array}$ & & $\begin{array}{l}-0.007 \\
(0.017)\end{array}$ & $\begin{array}{l}-0.000 \\
(0.001)\end{array}$ & & $\begin{array}{l}-0.002 \\
(0.003)\end{array}$ & $\begin{array}{l}-0.000 \\
(0.001)\end{array}$ & & $\begin{array}{l}-0.002 \\
(0.003)\end{array}$ \\
\hline Year 2015 & $\begin{array}{c}0.177 \\
(0.116)\end{array}$ & $\begin{array}{c}0.226^{*} \\
(0.134)\end{array}$ & $\begin{array}{c}0.172 \\
(0.122)\end{array}$ & $\begin{array}{l}0.231^{* * *} \\
(0.114)\end{array}$ & $\begin{array}{c}0.187 \\
(0.125)\end{array}$ & $\begin{array}{l}0.213^{*} \\
(0.119)\end{array}$ & $\begin{array}{c}0.127^{* * * *} \\
(0.019)\end{array}$ & $\begin{array}{c}0.126^{* * *} \\
(0.023)\end{array}$ & $\begin{array}{l}0.124^{* * * *} \\
(0.021)\end{array}$ & $\begin{array}{l}0.136^{* * * *} \\
(0.019)\end{array}$ & $\begin{array}{c}0.135^{* * *} \\
(0.021)\end{array}$ & $\begin{array}{c}0.133^{* * *} \\
(0.021)\end{array}$ \\
\hline Migrant (dummy) & $\begin{array}{c}0.089 \\
(0.159)\end{array}$ & $\begin{array}{c}0.177 \\
(0.229)\end{array}$ & $\begin{array}{c}0.228 \\
(0.227)\end{array}$ & $\begin{array}{c}0.100 \\
(0.160)\end{array}$ & $\begin{array}{c}0.174 \\
(0.229)\end{array}$ & $\begin{array}{c}0.234 \\
(0.227)\end{array}$ & $\begin{array}{l}-0.049^{*} \\
(0.027)\end{array}$ & $\begin{array}{l}-0.025 \\
(0.039)\end{array}$ & $\begin{array}{l}-0.029 \\
(0.039)\end{array}$ & $\begin{array}{l}-0.046^{*} \\
(0.027)\end{array}$ & $\begin{array}{l}-0.024 \\
(0.039)\end{array}$ & $\begin{array}{l}-0.028 \\
(0.039)\end{array}$ \\
\hline Masaka (dummy) & $\begin{array}{l}1.043^{* * *} \\
(0.144)\end{array}$ & & $\begin{array}{l}1.050^{* * * *} \\
(0.145)\end{array}$ & $\begin{array}{l}1.093^{* * *} \\
(0.143)\end{array}$ & & $\begin{array}{l}1.091^{* * * *} \\
(0.144)\end{array}$ & $\begin{array}{c}0.037 \\
(0.025)\end{array}$ & & $\begin{array}{c}0.029 \\
(0.025)\end{array}$ & $\begin{array}{l}0.043^{*} \\
(0.025)\end{array}$ & & $\begin{array}{c}0.037 \\
(0.025)\end{array}$ \\
\hline Constant & $\begin{array}{l}7.534^{* * * *} \\
(0.388)\end{array}$ & $\begin{array}{c}9.577^{* * *} \\
(0.992)\end{array}$ & $\begin{array}{l}7.633^{* * * *} \\
(0.425)\end{array}$ & $\begin{array}{l}7.722^{* * * *} \\
(0.386)\end{array}$ & $\begin{array}{c}9.378^{* * * *} \\
(0.982)\end{array}$ & $\begin{array}{l}7.773^{\text {**** }} \\
(0.424)\end{array}$ & $\begin{array}{c}0.464^{* * * *} \\
(0.067)\end{array}$ & $\begin{array}{l}0.373^{* *} \\
(0.168)\end{array}$ & $\begin{array}{c}0.468^{* * * *} \\
(0.074)\end{array}$ & $\begin{array}{c}0.482^{* * * *} \\
(0.066)\end{array}$ & $\begin{array}{l}0.418^{* *} \\
(0.166)\end{array}$ & $\begin{array}{c}0.484^{* * * *} \\
(0.074)\end{array}$ \\
\hline Observations & 874 & 874 & 874 & 874 & 874 & 874 & 874 & 874 & 874 & 874 & 874 & 874 \\
\hline No. households & 480 & 480 & 480 & 480 & 480 & 480 & 480 & 480 & 480 & 480 & 480 & 480 \\
\hline Wald $\chi^{2} / F$-value & $348.38^{* * *}$ & 0.83 & $389.61^{* * * *}$ & $343.58^{* * * *}$ & $0.71^{* * *}$ & $388.44^{* * * *}$ & $98.35^{* * * *}$ & $9.27^{* * * *}$ & $101.33^{* * *}$ & $93.95^{* * * *}$ & $8.97^{* * * *}$ & $97.13^{* * *}$ \\
\hline Hausman $\chi 2$ & & $14.84^{* *}$ & & & $14.82^{* *}$ & & & 3.04 & & & 3.50 & \\
\hline
\end{tabular}

Notes: Coefficient estimates are shown with standard errors in parentheses. RE, random effects estimator; FE, fixed effects estimator; MK, Mundlak estimator; FMP, mobile phone used by at least one female; MMP; mobile phone only used by males; AE, adult equivalents. ${ }^{* * * *} \mathrm{p}<0.01,{ }^{* *} \mathrm{p}<0.05,{ }^{*} \mathrm{p}<0.1$ 
Table 2.7: Effects of female and male mobile phone use on household nutrition

\begin{tabular}{|c|c|c|c|c|c|c|c|c|c|c|c|c|}
\hline & \multicolumn{6}{|c|}{ DDS 12 food groups } & \multicolumn{6}{|c|}{ DDS 9 food groups } \\
\hline & (1) RE & (2) FE & (3) MK & (4) RE & (5) FE & (6) MK & (7) RE & (8) FE & (9) MK & (10) RE & (11) FE & (12) MK \\
\hline $\begin{array}{l}\text { FMP use } \\
\text { (dummy) }\end{array}$ & $\begin{array}{c}0.350^{* * * *} \\
(0.112)\end{array}$ & $\begin{array}{c}0.034 \\
(0.246)\end{array}$ & $\begin{array}{c}0.310^{* * *} \\
(0.113)\end{array}$ & & & & $\begin{array}{c}0.307^{* * * *} \\
(0.095)\end{array}$ & $\begin{array}{c}0.102 \\
(0.214)\end{array}$ & $\begin{array}{c}0.277^{* * * *} \\
(0.096)\end{array}$ & & & \\
\hline $\begin{array}{l}\text { MMP use } \\
\text { (dummy) }\end{array}$ & & & & $\begin{array}{l}-0.198 \\
(0.125)\end{array}$ & $\begin{array}{c}0.196 \\
(0.245)\end{array}$ & $\begin{array}{l}-0.160 \\
(0.125)\end{array}$ & & & & $\begin{array}{c}-0.173 \\
(0.106)\end{array}$ & $\begin{array}{c}0.124 \\
(0.212)\end{array}$ & $\begin{array}{c}-0.143 \\
(0.107)\end{array}$ \\
\hline Education of & $0.056^{* * *}$ & $0.081^{*}$ & 0.044 & $0.062^{* * * *}$ & $0.078^{*}$ & 0.044 & $0.039^{* * *}$ & 0.061 & 0.029 & $0.044^{* * * *}$ & 0.059 & 0.029 \\
\hline head (years) & $(0.018)$ & $(0.043)$ & $(0.043)$ & $(0.018)$ & $(0.043)$ & $(0.044)$ & $(0.016)$ & $(0.037)$ & $(0.038)$ & $(0.016)$ & $(0.037)$ & $(0.038)$ \\
\hline Male head & $0.242^{*}$ & & $1.557^{* * * *}$ & 0.214 & & $1.577^{* * * *}$ & $0.220^{*}$ & & $1.340^{* * * *}$ & $0.195^{*}$ & & $1.357^{* * * *}$ \\
\hline (dummy) & $(0.137)$ & & $(0.432)$ & $(0.139)$ & & $(0.433)$ & $(0.116)$ & & $(0.375)$ & $(0.118)$ & & $(0.375)$ \\
\hline $\begin{array}{l}\text { Household size } \\
\text { (AE) }\end{array}$ & $\begin{array}{c}0.115^{* * *} \\
(0.023)\end{array}$ & $\begin{array}{c}0.081^{*} \\
(0.044)\end{array}$ & $\begin{array}{l}0.073^{*} \\
(0.044)\end{array}$ & $\begin{array}{c}0.125^{* * *} \\
(0.022)\end{array}$ & $\begin{array}{c}0.083^{*} \\
(0.044)\end{array}$ & $\begin{array}{c}0.069 \\
(0.044)\end{array}$ & $\begin{array}{c}0.079^{* * * *} \\
(0.019)\end{array}$ & $\begin{array}{c}0.059 \\
(0.038)\end{array}$ & $\begin{array}{c}0.052 \\
(0.038)\end{array}$ & $\begin{array}{c}0.088^{* * *} \\
(0.019)\end{array}$ & $\begin{array}{c}0.060 \\
(0.039)\end{array}$ & $\begin{array}{c}0.049 \\
(0.038)\end{array}$ \\
\hline Land owned (ha ) & $\begin{array}{c}0.035 \\
(0.042)\end{array}$ & & $\begin{array}{c}0.098 \\
(0.077)\end{array}$ & $\begin{array}{c}0.039 \\
(0.042)\end{array}$ & & $\begin{array}{c}0.096 \\
(0.077)\end{array}$ & $\begin{array}{l}0.068^{*} \\
(0.036)\end{array}$ & & $\begin{array}{l}0.129^{*} \\
(0.067)\end{array}$ & $\begin{array}{l}0.072^{* * *} \\
(0.036)\end{array}$ & & $\begin{array}{l}0.127^{*} \\
(0.067)\end{array}$ \\
\hline $\begin{array}{l}\text { Age of head } \\
\text { (years) }\end{array}$ & $\begin{array}{c}-0.009^{* * *} \\
(0.004)\end{array}$ & $\begin{array}{c}0.011 \\
(0.013)\end{array}$ & $\begin{array}{l}-0.005 \\
(0.014)\end{array}$ & $\begin{array}{c}-0.011^{* * * *} \\
(0.004)\end{array}$ & $\begin{array}{c}0.012 \\
(0.013)\end{array}$ & $\begin{array}{l}-0.006 \\
(0.014)\end{array}$ & $\begin{array}{c}-0.007^{* *} \\
(0.003)\end{array}$ & $\begin{array}{c}0.003 \\
(0.011)\end{array}$ & $\begin{array}{l}-0.010 \\
(0.012)\end{array}$ & $\begin{array}{c}-0.009^{* * *} \\
(0.003)\end{array}$ & $\begin{array}{c}0.003 \\
(0.011)\end{array}$ & $\begin{array}{l}-0.012 \\
(0.012)\end{array}$ \\
\hline $\begin{array}{l}\text { Distance to } \\
\text { tarmac road }(\mathrm{km})\end{array}$ & $\begin{array}{c}0.003 \\
(0.006)\end{array}$ & & $\begin{array}{c}0.004 \\
(0.014)\end{array}$ & $\begin{array}{c}0.001 \\
(0.006)\end{array}$ & & $\begin{array}{c}0.005 \\
(0.014)\end{array}$ & $\begin{array}{c}0.004 \\
(0.005)\end{array}$ & & $\begin{array}{c}0.006 \\
(0.012)\end{array}$ & $\begin{array}{c}0.003 \\
(0.005)\end{array}$ & & $\begin{array}{c}0.007 \\
(0.012)\end{array}$ \\
\hline Year 2015 & $\begin{array}{c}-0.276^{* * * *} \\
(0.096)\end{array}$ & $\begin{array}{c}-0.302^{* * * *} \\
(0.111)\end{array}$ & $\begin{array}{c}-0.285^{* * * *} \\
(0.102)\end{array}$ & $\begin{array}{l}-0.217^{* *} \\
(0.094)\end{array}$ & $\begin{array}{c}-0.292^{* * * *} \\
(0.104)\end{array}$ & $\begin{array}{c}-0.229^{* *} \\
(0.100)\end{array}$ & $\begin{array}{c}-0.436^{* * * *} \\
(0.083)\end{array}$ & $\begin{array}{c}-0.450^{* * * *} \\
(0.097)\end{array}$ & $\begin{array}{l}-0.425^{* * * *} \\
(0.0883)\end{array}$ & $\begin{array}{c}-0.383^{* * * *} \\
(0.081)\end{array}$ & $\begin{array}{c}-0.431^{* * *} \\
(0.091)\end{array}$ & $\begin{array}{c}-0.375^{* * * * *} \\
(0.087)\end{array}$ \\
\hline Migrant (dummy) & $\begin{array}{c}-0.271^{* *} \\
(0.131)\end{array}$ & $\begin{array}{c}-0.473^{* *} \\
(0.192)\end{array}$ & $\begin{array}{c}-0.412^{* * *} \\
(0.190)\end{array}$ & $\begin{array}{c}-0.259^{* * *} \\
(0.132)\end{array}$ & $\begin{array}{c}-0.473^{* *} \\
(0.191)\end{array}$ & $\begin{array}{c}-0.404^{* *} \\
(0.190)\end{array}$ & $\begin{array}{c}-0.238^{* *} \\
(0.112)\end{array}$ & $\begin{array}{c}-0.407^{* *} \\
(0.166)\end{array}$ & $\begin{array}{c}-0.351^{* *} \\
(0.165)\end{array}$ & $\begin{array}{c}-0.227^{* *} \\
(0.113)\end{array}$ & $\begin{array}{c}-0.406^{* *} \\
(0.166)\end{array}$ & $\begin{array}{c}-0.344^{* *} \\
(0.165)\end{array}$ \\
\hline Masaka (dummy) & $\begin{array}{l}-0.113 \\
(0.117)\end{array}$ & & $\begin{array}{l}-0.085 \\
(0.119)\end{array}$ & $\begin{array}{l}-0.061 \\
(0.117)\end{array}$ & & $\begin{array}{l}-0.036 \\
(0.119)\end{array}$ & $\begin{array}{l}-0.127 \\
(0.099)\end{array}$ & & $\begin{array}{l}-0.104 \\
(0.101)\end{array}$ & $\begin{array}{l}-0.081 \\
(0.099)\end{array}$ & & $\begin{array}{l}-0.060 \\
(0.101)\end{array}$ \\
\hline Constant & $\begin{array}{l}8.680^{* * * *} \\
(0.317) \\
\end{array}$ & $\begin{array}{l}7.957^{* * *} \\
(0.828) \\
\end{array}$ & $\begin{array}{l}8.843^{* * * *} \\
(0.349) \\
\end{array}$ & $\begin{array}{l}8.872^{* * * *} \\
(0.317) \\
\end{array}$ & $\begin{array}{l}7.904^{* * * *} \\
(0.817) \\
\end{array}$ & $\begin{array}{l}8.991^{* * * *} \\
(0.350)\end{array}$ & $\begin{array}{c}6.257^{* * * *} \\
(0.269) \\
\end{array}$ & $\begin{array}{c}6.065^{* * * *} \\
(0.718) \\
\end{array}$ & $\begin{array}{l}6.335^{* * * *} \\
(0.295) \\
\end{array}$ & $\begin{array}{c}6.425^{* * * *} \\
(0.269) \\
\end{array}$ & $\begin{array}{c}6.083^{* * * *} \\
(0.709) \\
\end{array}$ & $\begin{array}{c}6.467^{* * * *} \\
(0.296) \\
\end{array}$ \\
\hline Observations & 874 & 874 & 874 & 874 & 874 & 874 & 874 & 874 & 874 & 874 & 874 & 874 \\
\hline No. households & 480 & 480 & 480 & 480 & 480 & 480 & 480 & 480 & 480 & 480 & 480 & 480 \\
\hline $\begin{array}{l}\text { Wald } \chi^{2} \text { / } F \text {-val. } \\
\text { Hausman } \chi 2\end{array}$ & $98.07^{* * * *}$ & $\begin{array}{l}3.33^{* * *} \\
7.69\end{array}$ & $115.66^{* * *}$ & $89.83^{* * * *}$ & $\begin{array}{l}3.43^{* * * *} \\
10.06\end{array}$ & $108.90^{* * * *}$ & $102.28^{* * *}$ & $\begin{array}{l}5.52^{* * * *} \\
4.55\end{array}$ & $117.06^{* * *}$ & $93.66^{* * * *}$ & $\begin{array}{l}5.54^{* * *} \\
6.75\end{array}$ & $109.78^{* * *}$ \\
\hline
\end{tabular}

Notes: Coefficient estimates are shown with standard errors in parentheses. DDS, dietary diversity score; RE, random effects estimator; FE, fixed effects estimator; MK, Mundlak estimator; FMP, mobile phone used by at least one female; MMP; mobile phone only used by males; $\mathrm{AE}$, adult equivalents. ${ }^{* * *} \mathrm{p}<0.01,{ }^{* *} \mathrm{p}<0.05,{ }^{*} \mathrm{p}<0.1$ 


\subsubsection{Impact Pathways}

We now turn to estimating the simultaneous equation model explained in equations (2.4) to (2.7) in order to test the expected causal pathways more explicitly. The system of equations can be estimated with aggregate mobile phone use (MP) or also with female mobile phone use (FMP) and male mobile phone use (MMP) separately. The previous sections showed that FMP is more important for the economic and social effects analyzed here; hence we only show the results using the FMP dummy. The main effects are summarized in Table 2.8. Full effects are shown in Table 2.11 (appendix 2 of supplementary material).

Table 2.8: Effects of female mobile phone use (summary of causal pathways)

\begin{tabular}{lcc}
\hline & DDS 12 food groups & $(2)$ \\
& & DDS 9 food groups \\
\hline Effect on DDS & $0.335^{*}$ & $0.333^{* *}$ \\
Household income (million UGX) & $(0.174)$ & $(0.150)$ \\
Gender equality (proportion of assets) & $2.451^{* *}$ & 1.473 \\
& $(1.067)$ & $(0.915)$ \\
Effect on household income (million UGX) & $0.455^{* *}$ & $0.466^{* *}$ \\
FMP use (dummy) & $(0.222)$ & $(0.220)$ \\
Effect on gender equality (proportion of asset) & $0.065^{* * *}$ & $0.065^{* * *}$ \\
FMP use (dummy) & $(0.022)$ & $(0.022)$ \\
& & $0.025^{* * *}$ \\
Effect on FMP use (dummy) & $0.025^{* * *}$ & $(0.006)$ \\
Neighbors using mobile phone & $(0.006)$ & $0.391^{* * *}$ \\
Network coverage & $0.391^{* * *}$ & $(0.048)$ \\
\hline
\end{tabular}

Notes: Estimates from two separate simultaneous equation systems are summarized in columns (1) and (2). Coefficient estimates are shown with standard errors in parentheses. Full model results are shown in Table 2.11 (supplementary online material). DDS, dietary diversity score; FMP, mobile phone used by at least one female, ${ }^{* * *}$ $\mathrm{p}<0.01,{ }^{* *} \mathrm{p}<0.05,{ }^{*} \mathrm{p}<0.1$

Column (1) of Table 2.8 shows results of the model with the 12 food group DDS as the final outcome variable. As can be seen, household income and gender equality both have positive and significant effects, meaning that they increase DDS and thus food security. A one million UGX (372 US\$) increase in annual household income will lead to an increase in dietary diversity by 
0.34 food groups. The coefficient of gender equality is larger, which is also due to the fact that this variable is measured as the proportion of assets owned. When all productive assets are owned by females or jointly by males and females in the household (as opposed to ownership by males only), the number of food groups consumed in the household will increase by 2.45 . This is a very substantial effect, clearly underlining the importance of women empowerment for food security.

The middle part in column (1) of Table 2.8 shows that both variables - household income and gender equality - are positively affected by female mobile phone use. Thus, the simultaneous equation model confirms that the effects of mobile phones on nutrition are channeled through household income and gender equality. We can also use the coefficient estimates in Table 2.8 to calculate the relative contribution of each of these two pathways. The effect of female mobile phone use on DDS through the income pathway is $0.455 \times 0.335=0.152$, whereas the effect through the gender equality pathway is $0.065 \times 2.451=0.159$. Hence, female mobile phone use contributes to improved nutrition almost equally through both pathways. And the combined effect of $0.152+0.159=0.311$ is similar in magnitude to the directly estimated effect of female mobile phone use on DDS of 0.350 in the reduced-form model in Table 2.7. In other words, the two pathways modeled here seem to explain most of the effects of mobile phones on household food security.

The lowest part of column (1) in Table 2.8 shows that the two instruments employed for female mobile phone use - number of neighbors using mobile phones and network coverage - are highly significant. The consistency of this instrumental variable approach with the earlier results confirms the robustness of the findings and also suggests that reverse causality is not an issue.

Column (2) of Table 2.8 shows estimates for the same simultaneous equation model but now using the 9 food group DDS as the final outcome variable. The results are very similar to those in 
column (1), only that the gender equality effect on DDS is smaller and statistically insignificant. This model suggests that income is more important for dietary quality than women empowerment. However, concluding that gender equality would not matter at all would be wrong. When excluding income from this model, the coefficient of gender equality increases and turns significant, meaning that income and gender equality are positively correlated.

\subsection{Conclusions}

Mobile phone technologies have spread very rapidly in rural Africa and other parts of the developing world. While previous studies had analyzed effects of mobile phone use on economic indicators - such as input and output prices, profits, and income - research on implications for broader social development is scarce. Better understanding social welfare effects is of particular importance against the background of the United Nations' Sustainable Development Goals. In this paper, we have used data from farm households in Uganda to analyze effects of mobile phone use on household income, gender equality, and nutrition. Gender equality was measured in terms of the proportion of household productive assets owned by females or jointly by female and male household members, as opposed to ownership by male members alone. Nutrition was measured in terms of two dietary diversity scores that portray food security and dietary quality.

Results from reduced-form panel regressions showed that mobile phone use has positive and significant effects on income and gender equality. After controlling for other factors, mobile phone use has increased household income by $26 \%$ and gender equality by $19 \%$. Likewise, mobile phone use has enhanced household food security and dietary quality. Gender disaggregation further revealed that female mobile phone use has stronger positive effects than male mobile phone use alone. Women seem to benefit over-proportionally from mobile phone technologies, which is plausible given that women are often particularly constrained in their 
access to markets and information. Hence, a new technology that helps reduce transaction costs and allows new forms of communication can be particularly advantageous for women. Higher incomes and better access to information for women influence their bargaining position within the household, thus also improving gender equality.

Simultaneous equation models were used to analyze causal pathways of these effects more explicitly. Estimation results showed that the effects of mobile phones on household diets are primarily channeled through the income and gender equality pathways. Both variables significantly affect dietary diversity, and both variables are positively affected by mobile phone use. In terms of relative magnitudes, both channels play almost equal roles for the nutrition effects of mobile phone technologies. In the simultaneous equation models, we also used instrumental variables to explain mobile phone adoption, thus controlling for reverse causality and other possible endogeneity issues. The similarity of the results between the reduced-form and the simultaneous equation models underlines the robustness of the findings.

Nevertheless, a few limitations are worth highlighting. First, our results refer to the specific setting in Uganda and cannot be generalized without further analysis in other regions. Second, our panel data only include two survey rounds. While a panel has clear advantages over crosssection data, more survey rounds would be useful for increasing the reliability of the impact estimates and for better capturing possible long-term effects. Third, and related to the previous point, our panel survey covers a time span in which many of the rural households in Uganda had already adopted mobile phones. Adoption rates further increased between the first and the second survey round, but it is possible that an earlier baseline survey with lower adoption rates would have led to somewhat different results. Unfortunately, such baseline data were not available. In spite of these limitations, we cautiously conclude that mobile phone technologies can improve 
gender equality and nutrition in rural households, especially when women have access to mobile phones. Gender-sensitive promotion strategies will have to ensure that these potentials are realized at large scale. 


\section{Appendix 2: Supplementary Material}

Table 0.19: Socioeconomic characteristics by female mobile phone use (FMP)

\begin{tabular}{lcccccc}
\hline & \multicolumn{2}{c}{2012} & \multicolumn{2}{c}{2015} & \multicolumn{2}{c}{ Pooled sample } \\
\cline { 2 - 7 } & $\begin{array}{c}\text { Non-users } \\
(\mathrm{N}=217)\end{array}$ & $\begin{array}{c}\text { Users } \\
(\mathrm{N}=202)\end{array}$ & $\begin{array}{c}\text { Non-users } \\
(\mathrm{N}=160)\end{array}$ & $\begin{array}{c}\text { Users } \\
(\mathrm{N}=295)\end{array}$ & $\begin{array}{c}\text { Non-users } \\
(\mathrm{N}=377)\end{array}$ & $\begin{array}{c}\text { Users } \\
(\mathrm{N}=497)\end{array}$ \\
\hline Age of household head (years) & 53.304 & 51.817 & 55.363 & 54.498 & 54.178 & 53.408 \\
& $(15.021)$ & $(13.503)$ & $(14.955)$ & $(13.173)$ & $(15.008)$ & $(13.359)$ \\
Education of household head & 5.816 & $7.381^{* * *}$ & 5.925 & $7.244^{* * *}$ & 5.862 & $7.299^{* * * *}$ \\
(years) & $(3.248)$ & $(3.838)$ & $(3.299)$ & $(3.782)$ & $(3.266)$ & $(3.801)$ \\
Male household head (dummy) & 0.802 & $0.708^{* *}$ & 0.844 & $0.742^{* *}$ & 0.819 & $0.728^{* * *}$ \\
Migrant household (dummy) & 0.212 & 0.223 & 0.094 & $0.176^{* *}$ & 0.162 & 0.195 \\
Household size (AE) & 4.879 & $5.501^{* *}$ & 4.448 & $5.547^{* * *}$ & 4.696 & $5.528^{* * * *}$ \\
& $(2.444)$ & $(3.074)$ & $(2.328)$ & $(2.638)$ & $(2.402)$ & $(2.820)$ \\
Land owned (hectares) & 2.236 & 2.319 & 2.273 & 2.395 & 2.252 & 2.364 \\
& $(1.726)$ & $(1.783)$ & $(4.132)$ & $(2.295)$ & $(2.989)$ & $(2.101)$ \\
Distance to tarmac road (km) & 17.058 & 15.565 & 16.354 & 14.947 & 16.759 & $15.198^{* *}$ \\
& $(11.718)$ & $(11.149)$ & $(10.356)$ & $(9.983)$ & $(11.151)$ & $(10.466)$ \\
Residence in Masaka & 0.447 & $0.549^{* * *}$ & 0.369 & $0.505^{* * * *}$ & 0.414 & $0.523^{* * * *}$ \\
Neighbors using mobile phone & 4.104 & $7.099^{* * *}$ & 6.700 & $9.208^{* * *}$ & 5.206 & $8.351^{* * * *}$ \\
& $(3.573)$ & $(2.802)$ & $(3.976)$ & $(1.802)$ & $(3.958)$ & $(2.486)$ \\
Network coverage & 0.479 & $0.950^{* * *}$ & 0.675 & $0.959^{* * *}$ & 0.562 & $0.956^{* * * *}$ \\
\hline
\end{tabular}

Notes: Mean values are shown with standard deviations in parentheses. AE, adult equivalents. Differences in means between users and non-users are tested for statistical significance. ${ }^{* * *} \mathrm{p}<0.01,{ }^{* *} \mathrm{p}<0.05,{ }^{*} \mathrm{p}<0.1$ 
Table 0.210: Socioeconomic characteristics by male mobile phone use (MMP)

\begin{tabular}{lcccccc}
\hline & \multicolumn{2}{c}{2012} & \multicolumn{2}{c}{2015} & \multicolumn{2}{c}{ Pooled sample } \\
\cline { 2 - 7 } & $\begin{array}{c}\text { Non-users } \\
(\mathrm{N}=314)\end{array}$ & $\begin{array}{c}\text { Users } \\
(\mathrm{N}=105)\end{array}$ & $\begin{array}{c}\text { Non-users } \\
(\mathrm{N}=343)\end{array}$ & $\begin{array}{c}\text { Users } \\
(\mathrm{N}=112)\end{array}$ & $\begin{array}{c}\text { Non-users } \\
(\mathrm{N}=148)\end{array}$ & $\begin{array}{c}\text { Users } \\
(\mathrm{N}=726)\end{array}$ \\
\hline Age of household head (years) & 53.429 & $50.067^{* * *}$ & 55.489 & $52.696^{*}$ & 54.505 & $51.424^{* * *}$ \\
& $(14.148)$ & $(14.569)$ & $(13.472)$ & $(14.679)$ & $(13.827)$ & $(14.651)$ \\
Education of household head & 6.579 & 6.543 & 6.918 & 6.357 & 6.757 & 6.447 \\
(years) & $(3.866)$ & $(2.804)$ & $(3.808)$ & $(3.190)$ & $(3.837)$ & $(3.004)$ \\
Male household head (dummy) & 0.688 & $0.962^{* * *}$ & 0.720 & $0.955^{* * *}$ & 0.705 & $0.959^{* * * *}$ \\
Migrant household (dummy) & 0.229 & 0.181 & 0.160 & 0.107 & 0.193 & $0.143^{*}$ \\
Household size (AE) & 5.131 & 5.323 & 5.219 & 4.982 & 5.177 & 5.147 \\
& $(2.885)$ & $(2.445)$ & $(2.683)$ & $(2.258)$ & $(2.779)$ & $(2.351)$ \\
Land owned (hectares) & 2.256 & 2.336 & 2.307 & 2.488 & 2.283 & 2.414 \\
& $(1.848)$ & $(1.435)$ & $(2.177)$ & $(4.878)$ & $(2.025)$ & $(3.636)$ \\
Distance to tarmac road (km) & 16.066 & 17.153 & 15.083 & 16.541 & 15.553 & 16.837 \\
& $(10.822)$ & $(13.202)$ & $(10.089)$ & $(10.208)$ & $(10.449)$ & $(11.729)$ \\
Residence in Masaka & 0.462 & $0.600^{* * *}$ & 0.461 & 0.446 & 0.461 & 0.521 \\
Neighbors using mobile phone & 5.164 & $6.695^{* * *}$ & 8.112 & $8.982^{* * *}$ & 6.703 & $7.876^{* * *}$ \\
& $(3.643)$ & $(2.997)$ & $(3.264)$ & $(1.931)$ & $(3.749)$ & $(2.748)$ \\
Network coverage & 0.631 & $0.933^{* * *}$ & 0.828 & $0.955^{* * *}$ & 0.734 & $0.945^{* * *}$ \\
\hline
\end{tabular}

Notes: Mean values are shown with standard deviations in parentheses. AE, adult equivalents. Differences in means between users and non-users are tested for statistical significance. ${ }^{* * *} p<0.01,{ }^{* *} p<0.05,{ }^{*} p<0.1$ 
Table 0. $\underline{311}$ : Effects of female mobile phone use (causal pathways)

\begin{tabular}{|c|c|c|c|c|c|c|c|c|}
\hline & \multicolumn{4}{|c|}{ DD2 12 food groups } & \multicolumn{4}{|c|}{ DDS 9 food groups } \\
\hline & DDS & Income & $\begin{array}{l}\text { Gender } \\
\text { equality }\end{array}$ & FMP use & DDS & Income & $\begin{array}{l}\text { Gender } \\
\text { equality }\end{array}$ & FMP use \\
\hline $\begin{array}{l}\text { Gender equality } \\
\text { (proportion of assets) }\end{array}$ & $\begin{array}{l}2.451^{* *} \\
(1.067)\end{array}$ & & & & $\begin{array}{c}1.473 \\
(0.915)\end{array}$ & & & \\
\hline $\begin{array}{l}\text { Household income } \\
\text { (million UGX) }\end{array}$ & $\begin{array}{l}0.335^{*} \\
(0.174)\end{array}$ & & & & $\begin{array}{l}0.333^{* *} \\
(0.150)\end{array}$ & & & \\
\hline Age of household & -0.001 & $-0.023^{* * * *}$ & -0.001 & 0.001 & 0.001 & $-0.023^{* * *}$ & -0.001 & 0.001 \\
\hline $\begin{array}{l}\text { head } \\
\text { Education of }\end{array}$ & $\begin{array}{l}(0.006) \\
-0.011\end{array}$ & $\begin{array}{l}(0.008) \\
0.169^{* * *}\end{array}$ & $\begin{array}{c}(0.001) \\
0.006\end{array}$ & $\begin{array}{l}(0.001) \\
0.020^{* * * *}\end{array}$ & $\begin{array}{l}(0.005) \\
-0.023\end{array}$ & $\begin{array}{l}(0.008) \\
0.169^{* * *}\end{array}$ & $\begin{array}{c}(0.001) \\
0.006\end{array}$ & $\begin{array}{l}(0.001) \\
0.020^{* * *}\end{array}$ \\
\hline household head & $(0.039)$ & $(0.037)$ & $(0.004)$ & $(0.005)$ & $(0.034)$ & $(0.037)$ & $(0.004)$ & $(0.005)$ \\
\hline Land owned (ha) & $\begin{array}{l}-0.205 \\
(0.153)\end{array}$ & $\begin{array}{l}0.839^{* * *} \\
(0.087)\end{array}$ & $\begin{array}{l}-0.019^{* *} \\
(0.008)\end{array}$ & $\begin{array}{l}-0.005 \\
(0.012)\end{array}$ & $\begin{array}{l}-0.186 \\
(0.132)\end{array}$ & $\begin{array}{l}0.839^{* * *} \\
(0.087)\end{array}$ & $\begin{array}{l}-0.019^{* *} \\
(0.008)\end{array}$ & $\begin{array}{l}-0.005 \\
(0.012)\end{array}$ \\
\hline $\begin{array}{l}\text { Distance to tarmac } \\
\text { road }(\mathrm{km})\end{array}$ & $\begin{array}{c}0.004 \\
(0.007)\end{array}$ & $\begin{array}{l}-0.004 \\
(0.012)\end{array}$ & $\begin{array}{l}-0.0001 \\
(0.001)\end{array}$ & $\begin{array}{l}-0.002 \\
(0.002)\end{array}$ & $\begin{array}{c}0.005 \\
(0.006)\end{array}$ & $\begin{array}{l}-0.004 \\
(0.012)\end{array}$ & $\begin{array}{l}-0.000 \\
(0.001)\end{array}$ & $\begin{array}{l}-0.002 \\
(0.002)\end{array}$ \\
\hline House size (AE) & $\begin{array}{c}0.034 \\
(0.043)\end{array}$ & $\begin{array}{l}0.099^{* * *} \\
(0.046)\end{array}$ & & & $\begin{array}{c}0.018 \\
(0.038)\end{array}$ & $\begin{array}{l}0.099^{* *} \\
(0.046)\end{array}$ & & \\
\hline Male head (dummy) & $\begin{array}{c}0.153 \\
(0.191)\end{array}$ & $\begin{array}{l}0.605^{* * *} \\
(0.274)\end{array}$ & $\begin{array}{c}-0.070^{* * *} \\
(0.027)\end{array}$ & $\begin{array}{c}-0.267^{* * * *} \\
(0.036)\end{array}$ & $\begin{array}{c}0.078 \\
(0.165)\end{array}$ & $\begin{array}{l}0.609^{* *} \\
(0.274)\end{array}$ & $\begin{array}{c}-0.071^{* * * *} \\
(0.027)\end{array}$ & $\begin{array}{c}-0.267^{* * * *} \\
(0.036)\end{array}$ \\
\hline Masaka (dummy) & $\begin{array}{c}-0.634^{* *} \\
(0.289)\end{array}$ & $\begin{array}{l}1.345^{* * * *} \\
(0.232)\end{array}$ & $\begin{array}{c}0.035 \\
(0.023)\end{array}$ & $\begin{array}{c}0.059^{*} \\
(0.031)\end{array}$ & $\begin{array}{c}-0.612^{* * *} \\
(0.250)\end{array}$ & $\begin{array}{l}1.343^{* * *} \\
(0.232)\end{array}$ & $\begin{array}{c}0.035 \\
(0.023)\end{array}$ & $\begin{array}{c}0.059^{*} \\
(0.031)\end{array}$ \\
\hline Migrant (dummy) & $\begin{array}{l}-0.123 \\
(0.171)\end{array}$ & $\begin{array}{c}0.051 \\
(0.277)\end{array}$ & $\begin{array}{l}-0.055^{* *} \\
(0.027)\end{array}$ & & $\begin{array}{l}-0.154 \\
(0.148)\end{array}$ & $\begin{array}{l}0.050 \\
(0.277)\end{array}$ & $\begin{array}{l}-0.055^{* *} \\
(0.027)\end{array}$ & \\
\hline Year 2015 (dummy) & $\begin{array}{c}-0.597^{* * * *} \\
(0.196)\end{array}$ & $\begin{array}{c}0.075 \\
(0.217)\end{array}$ & $\begin{array}{l}0.126^{* * * *} \\
(0.021)\end{array}$ & $\begin{array}{c}0.037 \\
(0.031)\end{array}$ & $\begin{array}{c}-0.633^{* * * *} \\
(0.169)\end{array}$ & $\begin{array}{c}0.073 \\
(0.217)\end{array}$ & $\begin{array}{c}0.127^{* * * *} \\
(0.021)\end{array}$ & $\begin{array}{c}0.037 \\
(0.031)\end{array}$ \\
\hline FMP use (dummy) & & $\begin{array}{l}0.455^{* * *} \\
(0.222)\end{array}$ & $\begin{array}{c}0.065^{* * * *} \\
(0.022)\end{array}$ & & & $\begin{array}{l}0.466^{* *} \\
(0.220)\end{array}$ & $\begin{array}{l}0.065^{* * * *} \\
(0.022)\end{array}$ & \\
\hline $\begin{array}{l}\text { Neighbors using } \\
\text { mobile phone }\end{array}$ & & & & $\begin{array}{c}0.025^{* * * *} \\
(0.006)\end{array}$ & & & & $\begin{array}{l}0.025^{* * * *} \\
(0.006)\end{array}$ \\
\hline Network coverage & & & & $\begin{array}{l}0.391^{* * * *} \\
(0.048)\end{array}$ & & & & $\begin{array}{l}0.391^{* * * *} \\
(0.048)\end{array}$ \\
\hline House size (persons) & & & $\begin{array}{c}0.019^{* * *} \\
(0.004)\end{array}$ & $\begin{array}{c}0.016^{* * * *} \\
(0.005)\end{array}$ & & & $\begin{array}{c}0.019^{* * * *} \\
(0.004)\end{array}$ & $\begin{array}{c}0.016^{* * * *} \\
(0.005)\end{array}$ \\
\hline Constant & $\begin{array}{l}7.251^{* * *} \\
(0.701)\end{array}$ & $\begin{array}{c}1.069^{*} \\
(0.639)\end{array}$ & $\begin{array}{c}0.444^{* * * *} \\
(0.063)\end{array}$ & $\begin{array}{c}0.021 \\
(0.088) \\
\end{array}$ & $\begin{array}{c}5.273^{* * * *} \\
(0.604)\end{array}$ & $\begin{array}{c}1.064^{*} \\
(0.639) \\
\end{array}$ & $\begin{array}{c}0.444^{* * * *} \\
(0.063)\end{array}$ & $\begin{array}{c}0.021 \\
(0.088) \\
\end{array}$ \\
\hline Observations & 874 & 874 & 874 & 874 & 874 & 874 & 874 & 874 \\
\hline R-squared & -0.345 & 0.237 & 0.107 & 0.309 & -0.413 & 0.237 & 0.107 & 0.309 \\
\hline
\end{tabular}

Notes: Coefficient estimates are shown with standard errors in parentheses. DDS, dietary diversity score; FMP, mobile phone used by at least one female; AE, adult equivalents, ${ }^{* * *} \mathrm{p}<0.01,{ }^{* *} \mathrm{p}<0.05,{ }^{*} \mathrm{p}<0.1$ 


\section{Mobile Money, Agricultural Marketing, and Off-Farm Income}

\section{in Uganda ${ }^{2}$}

Abstract: Mobile money (MM) services can contribute to welfare gains in smallholder farm households. Previous research showed that one important pathway for these MM-related welfare gains is through higher remittances received from relatives and friends. Here, the role of other impact pathways is examined, especially focusing on agricultural marketing and off-farm economic activities. The analysis builds on panel data from smallholder coffee farmers in Uganda. Regression models show that the adoption of MM technology has contributed to higher household incomes and consumption levels. Off-farm income gains are identified to be an important pathway, also beyond remittances. Typical off-farm income sources are small businesses in trade, transport, and handicrafts, which benefit from novel savings and money transfer opportunities through MM. In terms of agricultural marketing, MM users sell a larger proportion of their coffee as shelled beans to buyers in high-value markets, instead of selling to local traders immediately after harvest. MM services help to reduce cash constraints and facilitate transactions with buyers from outside local regions. In conclusion, MM can contribute to rural development through various important pathways. Analysis of adoption patterns suggests that MM services are socially inclusive.

Keywords: mobile phones; rural banking; smallholder farmers; impact evaluation; Africa

\footnotetext{
2 This paper was co-authored with Matin Qaim, who assisted in shaping the research ideas through comments, and the final writing of the paper. I originated the ideas, designed the questionnaire, collected data, and analyzed it. Finally, I put together the paper draft on to which Matin Qaim provided comments, and relevant literature updates until the paper was published in the journal "Agricultural Economics".
} 


\subsection{Introduction}

The use of mobile phone technologies has rapidly increased in many developing countries since the late-1990s. This has contributed to economic growth and poverty reduction, especially in rural areas where mobile phones have helped households to access better market information and fetch higher prices for their products (Torero and von Braun, 2005; Jensen, 2007; Muto and Yamano, 2009; Aker, 2010; Aker, 2011; Aker and Mbiti, 2010; Sekabira et al., 2012; Nakasone et al., 2014; Tadesse and Bahiigwa, 2015; Aker and Ksoll, 2016; Blauw and Franses, 2016). In addition to the direct positive effects of mobile phones, their widespread use has also facilitated the adoption of other mobile technologies. One important example is mobile money (MM). MM services enable the electronic transfer of money via mobile phones. This reduces transaction costs for the payment of bills and for making remittances. Recipients of electronic transfers can either save the money on their mobile account or collect it in cash from a MM service center. MM services are particularly attractive for people with limited access to the traditional banking system. The recent spread of this technology was particularly rapid in sub-Saharan Africa (Suri et al., 2012; Jack et al., 2013).

MM could revolutionize the nature of market transactions and private transfers for the previously unbanked, but so far relatively little is known about the real effects in developing countries (Nakasone et al., 2014). Especially for smallholder farmers, the knowledge about MM effects is thin. A number of recent studies have looked at impacts on household welfare in Kenya and Uganda (Kirui et al., 2013; Jack and Suri, 2014; Kikulwe et al., 2014; Murendo and Wollni, 2016; Munyegera and Matsumoto, 2016). These studies confirm that MM has positive effects on income, consumption, and food security. However, the pathways through which MM affects these welfare outcomes remain understudied. One pathway that most studies mention is 
remittances. More remittances received from relatives and friends increase household income directly. Indirect effects can occur because remittances also act as a kind of insurance (Jack and Suri, 2014; Munyegera and Matsumoto, 2016). Wider effects for other economic activities of farm households have hardly been studied. One exception is Kikulwe et al. (2014) who showed that MM has increased the use of agricultural inputs and levels of commercialization in the Kenyan small farm sector.

We add to this literature by further analyzing how the adoption of MM technology affects economic activities of smallholder households, including both farm and off-farm activities. To our knowledge, impacts of MM on off-farm income of smallholder farmers have not been analyzed beyond the question of remittances. We hypothesize that the new options for savings as well as for transferring money between business partners may especially encourage selfemployed activities and thus increase off-farm income. Through similar mechanisms, agricultural income may increase as well. Here, we are particularly interested to see whether MM allows farmers to access high-value markets where better prices can be obtained. For the empirical analysis, we use panel data collected from coffee farmers in Uganda. Uganda is of interest not only because many of the poor are smallholder farmers, but also because MM technology has been rapidly adopted there in recent years.

\subsection{Conceptual Framework}

The use of mobile money (MM) services can influence the welfare of farm households in various ways. A simple framework of potential pathways is shown in Figure 3.1. A first pathway that was confirmed to be relevant in recent empirical work is higher remittances received from relatives

and friends (Suri et al., 2012; Jack and Suri, 2014; Munyegera and Matsumoto, 2016). The main reason for the increase in remittances is that $\mathrm{MM}$ has lowered the transaction costs of transferring 
money even to remote rural locations. As a source of income, remittances can contribute to improved household welfare directly. In addition, the higher availability of cash can facilitate investments into farm and off-farm economic activities. Remittances are often a more reliable source of income than self-employed activities for the rural poor, thus also providing some kind of insurance (Jack et al., 2013).

Figure 3.1: Impact pathways of mobile money use on household welfare

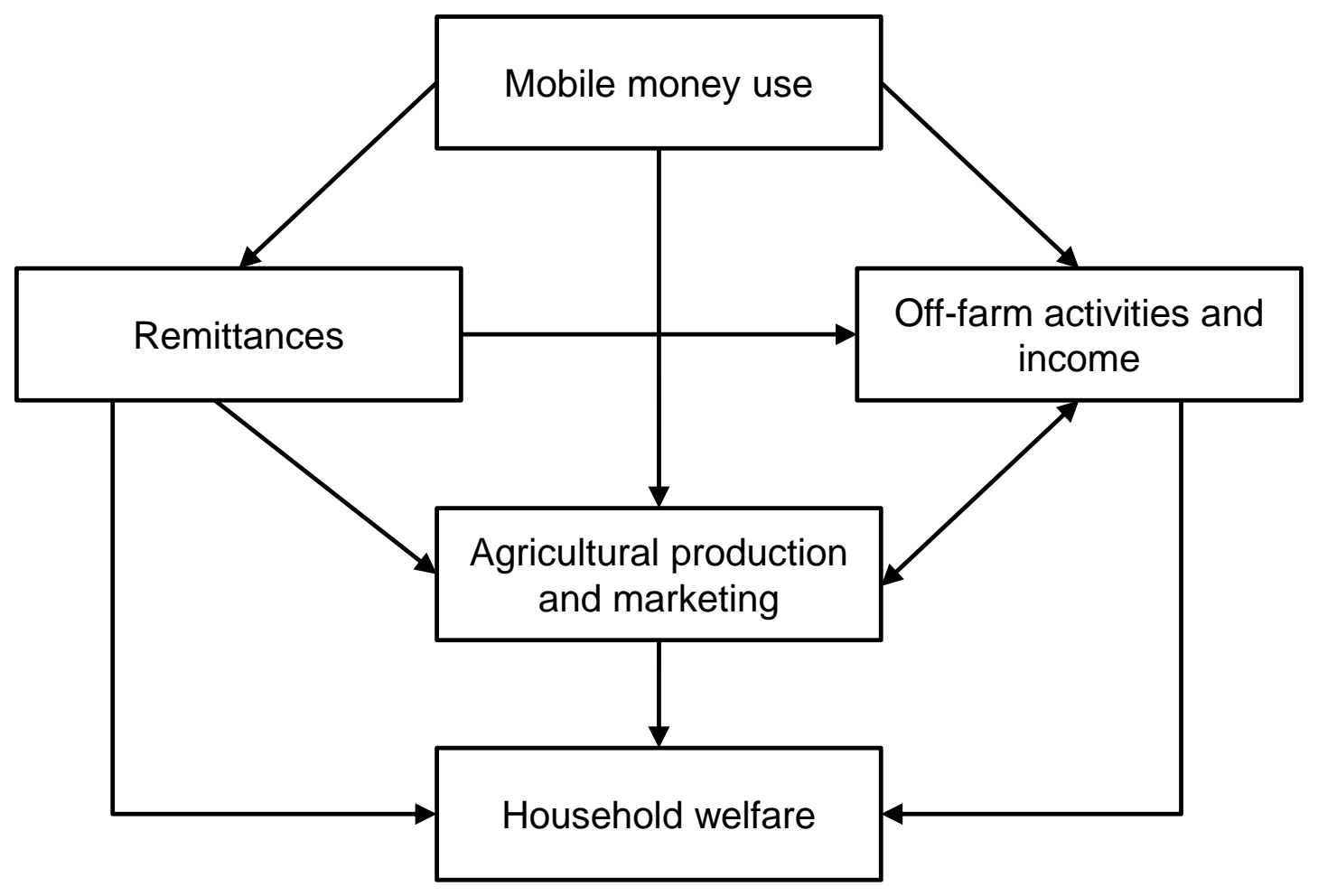

Use of MM can also affect farm and off-farm economic activities directly. People often use their MM account for savings. Savings on the MM account can be used for later cash withdrawals or for paying business partners for goods and services received. Kikulwe et al. (2014) showed that farmers with MM used more fertilizer, pesticides, and hired labor. MM adopters also marketed a larger proportion of their output. Especially when the ordering of goods and services, the 
delivery, and the payment do not occur in one place and at one point in time, MM transfers can be useful to reduce transaction costs. Such conditions are particularly relevant in high-value agricultural markets (Blandon et al., 2009; Reardon and Timmer, 2012; Andersson et al., 2015).

An important question for farmers is when to sell their crop, in what form, and to whom. Smallholders often sell their produce to local traders immediately after harvest, without any further storage or processing, because they need the cash to pay for urgent consumption needs or for outstanding bills (Fafchamps and Hill, 2005). We hypothesize that the use of MM allows farmers to sell a larger proportion of their crop after post-harvest processing. Related to this, we also hypothesize that MM helps farmers to fetch higher prices for their sales. This is not only related to higher levels of processing. Even at the same processing level, farmers with MM may find it easier to transact with buyers in different locations, thus being able to benefit from the best price offers.

Off-farm income sources also play an important role for many smallholder farmers. Beyond salaried employment, many households have their own small non-farm business, for instance in food processing, handicrafts, or transport, trade, and repair services. Such off-farm activities can also benefit from MM transactions. Off-farm income sources contribute directly to household welfare. In addition, off-farm earnings are sometimes used for investments in farming, especially in situations where rural financial markets fail (Oseni and Winters, 2009).

In the empirical analysis below, we analyze the impact of MM use on household welfare in terms of income and per capita consumption. We also examine some of the impact pathways, concentrating especially on those that were not studied previously, such as off-farm income and aspects of agricultural marketing and prices. 


\subsection{Materials and Methods}

\subsubsection{Survey of Farm Households}

We use panel data collected in two survey rounds from randomly selected coffee-growing households in Luwero and Masaka (now named Bukomansimbi) Districts, Central Uganda. The first survey round was conducted in 2012, the second round in 2015.

The two districts were chosen, as they are important production regions for Robusta coffee. Farmers in these regions do not grow Arabica coffee, which requires higher altitudes. Within the two districts, we purposively selected three locations with a high density of coffee farmers. In these locations, we randomly selected farmers based on lists provided by village and coffee cooperative leaders. Many of the sample farmers are members of cooperatives, while others are not. The first round of the survey covered 419 coffee-producing households. In the second round, the same households were targeted, however, some sample attrition occurred. We had to replace 25 farmers that we were unable to interview again (6\% attrition rate). These replacements were randomly sampled in the same locations. In addition, we increased the sample size to a total of 455 households in 2015. Additional households were also sampled randomly in the same locations. For the analysis, we use the unbalanced panel with 874 observations from 480 households.

In both survey rounds, we used a structured questionnaire for face-to-face interviews. The questionnaire focused on details of coffee production and marketing, other farm and non-farm economic activities, consumption, as well as socio-demographic and contextual details. Farm and off-farm income sources were captured for a period of one year prior to each survey round. One section of the questionnaire also asked for mobile phone and mobile money use. The section about mobile money was only included in the 2015 survey round, but it also covered mobile 
technology use in 2012 through recall questions. The question of particular importance for this analysis is whether the respondent's household had started using mobile money services already before 2012. With some assistance from the interviewers, such as reminding of important past events as a reference, respondents had no problems in answering this recall question.

\subsubsection{Modelling Mobile Money Adoption}

In a first step, we want to explain what factors influence whether or not farmers use MM services. This is modeled in a binary choice framework as follows:

$M M_{j t}=\alpha+\beta \mathbf{X}_{j t}+\gamma T_{t}+\varepsilon_{j t}$

where $M M_{j t}$ is a dummy dependent variable that takes a value of one if household $j$ used MM in year $t$, and zero otherwise. $\mathbf{X}_{j t}$ is a vector of household, farm, and contextual characteristics, and $T_{t}$ is a year dummy controlling for time fixed effects and taking a value of one for observations referring to 2015. $\alpha, \beta$, and $\gamma$ are parameters to be estimated, and $\varepsilon_{j t}$ is a random error term.

\subsubsection{Modelling Mobile Money Impacts}

Beyond explaining MM adoption, we want to evaluate impacts of adoption on household welfare and on intermediate outcomes to explain income pathways. We use panel regression models as follows:

$Y_{j t}=\theta+\phi M M_{j t}+\chi \mathbf{V}_{j t}+\delta T_{t}+\mu_{j t}$

Where $Y_{j t}$ is the outcome variable such as income, consumption, or coffee price received by household $j$ in yeart. $M M_{j t}$ is the treatment dummy. A positive coefficient $\phi$ would imply that 
MM use is positively associated with income or other outcome variables of interest. We control for household, farm, and contextual variables that may affect outcomes through including the vector $\mathbf{V}_{j t}$. In addition, we control for time fixed effects through the year dummy $T_{t} \cdot \theta, \chi$, and $\delta$ are other parameters to be estimated, and $\mu_{j t}$ is a random error term.

The model in equation (3.2) can be estimated with random effects (RE) panel estimator. However, $M M_{j t}$ may potentially be endogenous, which would lead to correlation with the error term and thus to biased estimates of $\phi$. A likely source of endogeneity is unobserved heterogeneity between mobile money adopters and non-adopters. If there are any unobserved factors that influence $M M_{j t}$ and $Y_{j t}$ simultaneously, the estimates would suffer from selection bias. Since farmers decide themselves whether or not to use MM, it is well possible that adopters and non-adopters differ in terms of unobserved characteristics. Similarly, it is possible that early MM adopters differ from later adopters. To test and control for selection bias, we use a fixed effects (FE) estimator, which is possible because we have sufficient variation in the treatment variable over time. FE estimators evaluate differences within households, so that any timeinvariant heterogeneity between adopters and non-adopters - regardless of observed or unobserved - is cancelled out (Cameron and Trivedi, 2005). For all outcome variables, we compare RE and FE estimates by means of a Hausman test. An insignificant test result implies that unobserved, time-invariant heterogeneity is not an issue. In that case, the RE estimator is reliable and more efficient. A significant Hausman test indicates that the FE model is preferred to reduce selection bias in the estimated treatment effect.

Unfortunately, FE estimators cannot control for time-variant unobserved heterogeneity, which may also contribute to endogeneity bias. For instance, it is possible that MM users are also faster 
in adopting other innovations, which - unless controlled for - may lead to overestimated treatment effects. We will carry out several robustness checks to test for the possible role of unobserved time-variant heterogeneity. Beyond including additional proxies for innovation adoption, we also use an instrumental variable (IV) approach. When an appropriate instrument is available, the IV approach is useful not only to test for unobserved heterogeneity, but also to correct for reverse causality, another possible source of endogeneity. Reverse causality could occur when MM adoption and household income affect each other. Details of these robustness checks are provided below in subsection 3.4.6.

\subsubsection{Variables used}

The treatment variable in all models is MM use, which is defined as a dummy that takes a value of one if at least one household member had a MM account and had used MM services in the respective year. In almost all adopting households, the household head is a MM user, even though other household members may have their own MM account as well.

Household welfare is measured in terms of two indicators, namely household income and per capita consumption. Household income is the combined farm and off-farm income obtained over a period of one year. Farm income includes the value of all farm produce - either sold or kept for household consumption - minus production costs. Off-farm income includes salaries, wages, and pensions of all household members, land rents and capital earnings, as well as any net profit (revenue minus cost) from non-agricultural businesses. Remittances are also included as an offfarm income source. The other welfare measure - per capita consumption - measures the value of all food and non-food goods and services consumed in the household divided by the number of persons living in the household. Food consumption data were collected through a seven-day food 
recall. For most non-food items, monthly expenditures were recorded. For the analysis, we converted all expenditure data to a daily basis.

Remittances and other off-farm incomes are used as intermediate outcome variables. Remittances refer to money received during the respective year from any relatives or friends not living in the same household. This can be through MM services or through any other mechanism. To differentiate between different types of off-farm income, we calculate off-farm income with and without remittances included.

To evaluate agricultural marketing pathways, we look at the proportion of coffee sold in semiprocessed form, namely as shelled green beans, which is the highest processing level that farmers in Uganda can achieve. A bit more background about coffee marketing in the study region may help to better motivate this outcome variable. Farmers generally have four different marketing options. First, they can sell their coffee to middlemen when it is still at the flowering stage in the field. This is associated with very low prices and is therefore chosen only when farmers are particularly cash-constrained before the harvest. Second, when the coffee turns red, it can be harvested and sold as fresh red cherries. Third, the red cherries can be dried in the sun and then sold as 'kiboko'. Local traders buying coffee directly from farmers are primarily interested in either fresh red cherries or kiboko (Chiputwa et al., 2015). Hence, these are the two most common forms in which farmers in the study region sell their coffee. Fourth, the sun-dried cherries can be shelled and sold as green beans. Shelled green beans can fetch significantly higher prices, but they are usually not purchased by local traders. When selling green beans, farmers have to transact with buyers from outside their location, including exporters located in 
Kampala. Such transactions are often facilitated through the coffee cooperatives. ${ }^{3}$ Against this background, the proportion of coffee sold as shelled green beans is used here as a proxy for selling in higher-value markets. Farmers in the study region have no contracts that would determine the buyer or the form of coffee sales prior to the harvest. That is, farmers' decisions about which marketing channel to use are based on current conditions.

Also related to the marketing pathway, we use the average coffee price received by farmers in the respective year as another intermediate outcome variable. Given that farmers sell their coffee in various forms (e.g., red cherries, kiboko, and shelled green beans), the prices reported in the survey are not directly comparable. For instance, $5 \mathrm{~kg}$ of red cherries or $2 \mathrm{~kg}$ of kiboko ${ }^{4}$ will typically result in only $1 \mathrm{~kg}$ of shelled green beans. To make prices comparable, we used appropriate weight conversion factors. This does not account for the actual cost of processing, which is mainly the opportunity cost of time. However, during the survey many farmers told us that the cost is less of an issue. The main reasons mentioned for not selling more coffee in highervalue form were pressing consumption needs such as payments for medical care, school fees, food, or fuel.

All monetary values are expressed in Ugandan shillings (UGX) (1 US\$ $=2,690$ UGX). To account for inflation and make monetary values comparable for the two survey rounds, 2012 data were adjusted to 2015 using the official consumer price index (UBOS, 2015).

\footnotetext{
${ }^{3}$ Until the early-1990s, all coffee farmers in Uganda were organized in cooperatives and the coffee could only be marketed through these cooperatives. However, the market was liberalized in 1991 (Chiputwa and Qaim, 2016). Today, some farmers are organized in cooperatives while others are not. Cooperative members can sell their coffee through the cooperatives, but they are also free to use other marketing channels and sell directly to traders. On the other hand, non-members of cooperatives can use most of the processing and marketing services offered by the cooperatives against a fee.

${ }^{4}$ Kiboko is dried cherries of coffee ready for shelling into high-value shelled green coffee beans
} 
For most of the regression models, the same vector of covariates is used, even though depending on the particular outcome - individual variables are sometimes added. The vector of covariates includes household characteristics, such as education, age, and gender of the household head, farm characteristics, such as land owned and the value of other productive assets, and spatial characteristics, such as distance to the next tarmac road and a district dummy.

\subsection{Results and Discussions}

Mobile money (MM) services were introduced in Uganda in 2009. The most important MM service providers are Mobile Telephone Network (MTN) and Airtel, which are both foreignowned private companies. Seventy-one percent of MM adopters in our sample had an MTN account in 2015, 28\% had an Airtel account. Table 3.1 shows how MM use developed in recent years among the sample households. Between 2012 and 2015, the share of households with MM more than doubled from $23 \%$ to $62 \%$. This increase was facilitated by the rapid spread of MM service centers. Typically, these service centers are kiosks or small shops where cash can be deposited or withdrawn from mobile accounts. The same shops also provide other mobile phone related products and services. While in 2012 only $17 \%$ of the sample households had a MM service center in their village, by 2015 this had increased to 54\%. Table 3.1 also shows the development of mobile phone usage among sample households, which increased from $76 \%$ in 2012 to $89 \%$ in 2015.

\section{Table 3.1: Mobile money use and distribution}

\begin{tabular}{lccc}
\hline & 2012 & 2015 & Pooled sample \\
\hline $\begin{array}{l}\text { Proportion of households using mobile } \\
\text { money }\end{array}$ & 0.23 & $0.62 * * *$ & 0.44 \\
$\begin{array}{l}\text { Proportion of households with mobile } \\
\text { money service center in their village }\end{array}$ & 0.17 & $0.54 * * *$ & 0.36 \\
$\begin{array}{l}\text { Proportion of households using mobile } \\
\text { phone }\end{array}$ & 0.76 & $0.89 * * *$ & 0.83 \\
\hline Note: $* * *$ differences in proportions between the two years are significantly different at the $1 \%$ level.
\end{tabular}


Figure 3.2 shows the most important MM activities used by sample households. In this graph, for each adopting household we only counted the most frequently performed activity, so the numbers add up to $100 \%$. More than two-thirds of the households reported that withdrawing money from their mobile account is the most important activity. Withdrawals can be from previous own cash deposits or from transfers through business partners or private remittances. Usually, small amounts are withdrawn. The mobile accounts are considered relatively secure for savings. Depositing money is free, whereas for withdrawals a small proportional fee is charged. Most households also use their mobile accounts for sending money and for paying goods and services, but in terms of frequency these other activities were reported less often.

\section{Figure 3.2: Most important mobile money activity}

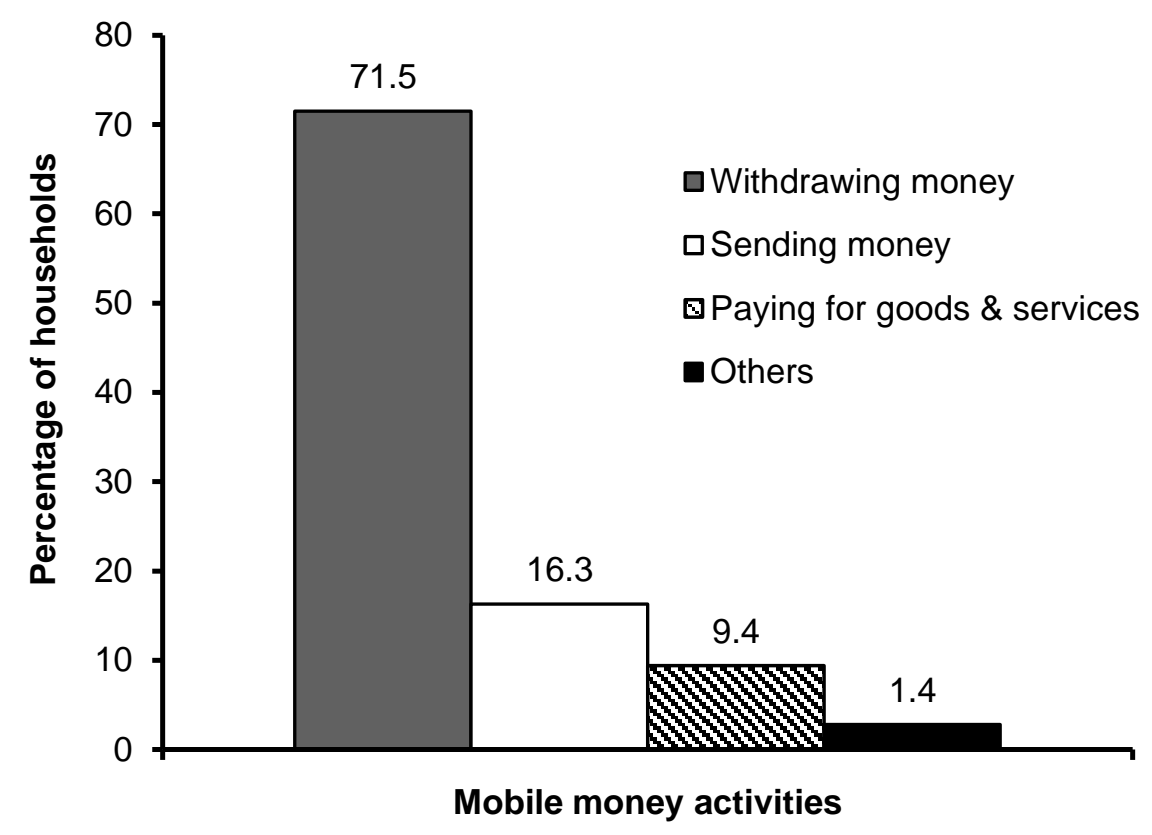




\subsubsection{Descriptive Statistics}

Table 3.2 presents descriptive statistics of the outcome variables and covariates used in the regression models, differentiating between MM users and non-users in 2012 and 2015.

Table 3.2: Descriptive statistics by users and non-users of mobile money

\begin{tabular}{|c|c|c|c|c|c|c|}
\hline & \multicolumn{2}{|c|}{2012} & \multicolumn{2}{|c|}{2015} & \multicolumn{2}{|c|}{ Pooled sample } \\
\hline & $\begin{array}{l}\text { Users } \\
(\mathrm{N}=98)\end{array}$ & $\begin{array}{c}\text { Non-users } \\
(\mathrm{N}=321)\end{array}$ & $\begin{array}{c}\text { Users } \\
(\mathrm{N}=284)\end{array}$ & $\begin{array}{c}\text { Non-users } \\
(\mathrm{N}=171)\end{array}$ & $\begin{array}{c}\text { Users } \\
(\mathrm{N}=382)\end{array}$ & $\begin{array}{c}\text { Non-users } \\
(\mathrm{N}=492)\end{array}$ \\
\hline \multicolumn{7}{|l|}{ Outcome variables } \\
\hline Household income (million & $3.754 * *$ & 2.876 & $4.186 * * *$ & 2.040 & $4.075 * * *$ & 2.585 \\
\hline UGX per year) & $(3.737)$ & $(3.173)$ & $(3.803)$ & $(2.260)$ & $(3.786)$ & $(2.913)$ \\
\hline Per capita consumption & 3.136 & 3.332 & 4.161 & 3.759 & $3.898 * * *$ & 3.481 \\
\hline (thousand. UGX per day) & $(1.645)$ & $(1.962)$ & $(2.714)$ & $(2.368)$ & $(2.522)$ & $(2.119)$ \\
\hline Remittances (million UGX per & 0.324 & 0.405 & 0.527 & 0.401 & 0.502 & 0.403 \\
\hline year) & $(0.499)$ & $(0.766)$ & $(0.596)$ & $(0.467)$ & $(0.623)$ & $(0.622)$ \\
\hline Off-farm income (million UGX & 1.013 & 0.813 & $1.421 * * *$ & 0.600 & $1.316^{* * *}$ & 0.739 \\
\hline per year) & $(1.533)$ & $(1.409)$ & $(1.748)$ & $(1.009)$ & $(1.703)$ & $(1.287)$ \\
\hline Off-farm income without & 0.960 & 0.750 & $1.209 * * *$ & 0.466 & $1.145 * * *$ & 0.651 \\
\hline remittances (million UGX) & $(1.496)$ & $(1.389)$ & $(1.694)$ & $(0.932)$ & $(1.647)$ & $(1.256)$ \\
\hline $\begin{array}{l}\text { Shelled coffee sales } \\
\text { (proportion) }\end{array}$ & 0.427 & 0.295 & $0.273 * * *$ & 0.099 & $0.313 *$ & 0.227 \\
\hline Coffee price (thousand. UGX & 4.478 & 4.446 & 4.288 & 4.217 & 4.350 & 4.401 \\
\hline per kg of shelled coffee) & $(0.465)$ & $(0.447)$ & $(0.245)$ & $(0.352)$ & $(0.342)$ & $(0.438)$ \\
\hline \multicolumn{7}{|l|}{ Explanatory variables } \\
\hline Education of household head & $5.945 * * *$ & 4.851 & $5.882 * * *$ & 4.469 & $5.898 * * *$ & 4.718 \\
\hline (years of schooling) & $(2.946)$ & $(3.388)$ & $(2.920)$ & $(3.199)$ & $(2.923)$ & $(3.325)$ \\
\hline \multirow[t]{2}{*}{ Age of household head (years) } & $54.118 *$ & 57.210 & $56.745 * * *$ & 61.989 & $56.071 * * *$ & 58.871 \\
\hline & $(11.577)$ & $(15.014)$ & $(13.018)$ & $(14.417)$ & $(12.701)$ & $(14.969)$ \\
\hline Male head (dummy) & 0.806 & 0.741 & $0.835 * * *$ & 0.684 & $0.827 * * *$ & 0.722 \\
\hline \multirow[t]{2}{*}{ Household size (persons) } & $7.534 * * *$ & 6.373 & $7.145 * * *$ & 5.448 & $7.245 * * *$ & 6.051 \\
\hline & $(3.145)$ & $(2.992)$ & $(2.907)$ & $(2.923)$ & $(2.970)$ & $(2.997)$ \\
\hline \multirow[t]{2}{*}{ Land owned (ha) } & $1.268 *$ & 1.007 & $1.131 * * *$ & 0.618 & $1.166^{* * *}$ & 0.872 \\
\hline & $(1.134)$ & $(1.172)$ & $(1.388)$ & $(1.394)$ & $(1.327)$ & $(1.266)$ \\
\hline Productive assets (million & $7.975 * * *$ & 7.258 & $8.028 * * *$ & 6.840 & $8.014 * * *$ & 7.113 \\
\hline UGX) & $(1.515)$ & $(1.799)$ & $(1.598)$ & $(1.747)$ & $(1.575)$ & $(1.790)$ \\
\hline \multirow[t]{2}{*}{ Distance to tarmac road $(\mathrm{km})$} & 17.888 & 18.322 & 17.900 & 17.282 & 17.897 & 17.961 \\
\hline & $(9.449)$ & $(10.145)$ & $(9.383)$ & $(9.297)$ & $(9.387)$ & $(9.862)$ \\
\hline Masaka district (dummy) & 0.500 & 0.495 & $0.493 * *$ & 0.398 & 0.495 & 0.461 \\
\hline Migrant household (dummy) & 0.224 & 0.215 & 0.158 & 0.129 & 0.175 & 0.185 \\
\hline Certified (dummy) & $0.745 * *$ & 0.617 & $0.673 *$ & 0.591 & $0.691 * *$ & 0.608 \\
\hline Neighbors using MM (number & $2.745^{* * *} *$ & 0.106 & $5.264 * * *$ & 0.111 & $4.618 * * *$ & 0.108 \\
\hline out of 10 nearest neighbors) & $(2.542)$ & $(0.686)$ & $(2.820)$ & $(0.723)$ & $(2.961)$ & $(0.698)$ \\
\hline \multirow[t]{2}{*}{ Age of coffee plants (years) } & $29.522 * *$ & 33.092 & 31.223 & 32.962 & $30.791 * * *$ & 33.047 \\
\hline & $(11.319)$ & $(13.443)$ & $(11.992)$ & $(12.793)$ & $(11.832)$ & $(13.208)$ \\
\hline Input use (thousand. UGX per & 49.802 & 44.323 & $68.123 * * *$ & 52.795 & $63.423 * * *$ & 47.267 \\
\hline ha) & $(31.652)$ & $(34.533)$ & $(31.435)$ & $(34.089)$ & $(32.454)$ & $(34.581)$ \\
\hline Walking time to coffee plots & 1.899 & 1.349 & $3.377 * * *$ & 2.465 & $2.998 * * *$ & 1.737 \\
\hline (minutes) & $(4.436)$ & $(4.444)$ & $(2.423)$ & $(2.104)$ & $(3.118)$ & $(3.832)$ \\
\hline
\end{tabular}

Notes: Mean values are shown with standard deviations in parentheses. $*, * *, * * *$ differences between MM-users and non-users are significant at the $10 \%, 5 \%$, and $1 \%$ level, respectively. 1 US $\$=2,690$ UGX 
Data for the pooled sample, including both survey rounds, are also shown in the last two table columns. MM users have higher household incomes and per capita consumption levels than nonusers. MM users also have higher off-farm incomes, both with and without remittances included. The most important off-farm income source for sample households are small businesses like retail shops, trade in forest products, transport services, or handicrafts, followed by remittances, and salaries from employment as teachers, nurses, or office clerks (Figure 3.3).

Figure 3.3: Most important off-farm income source

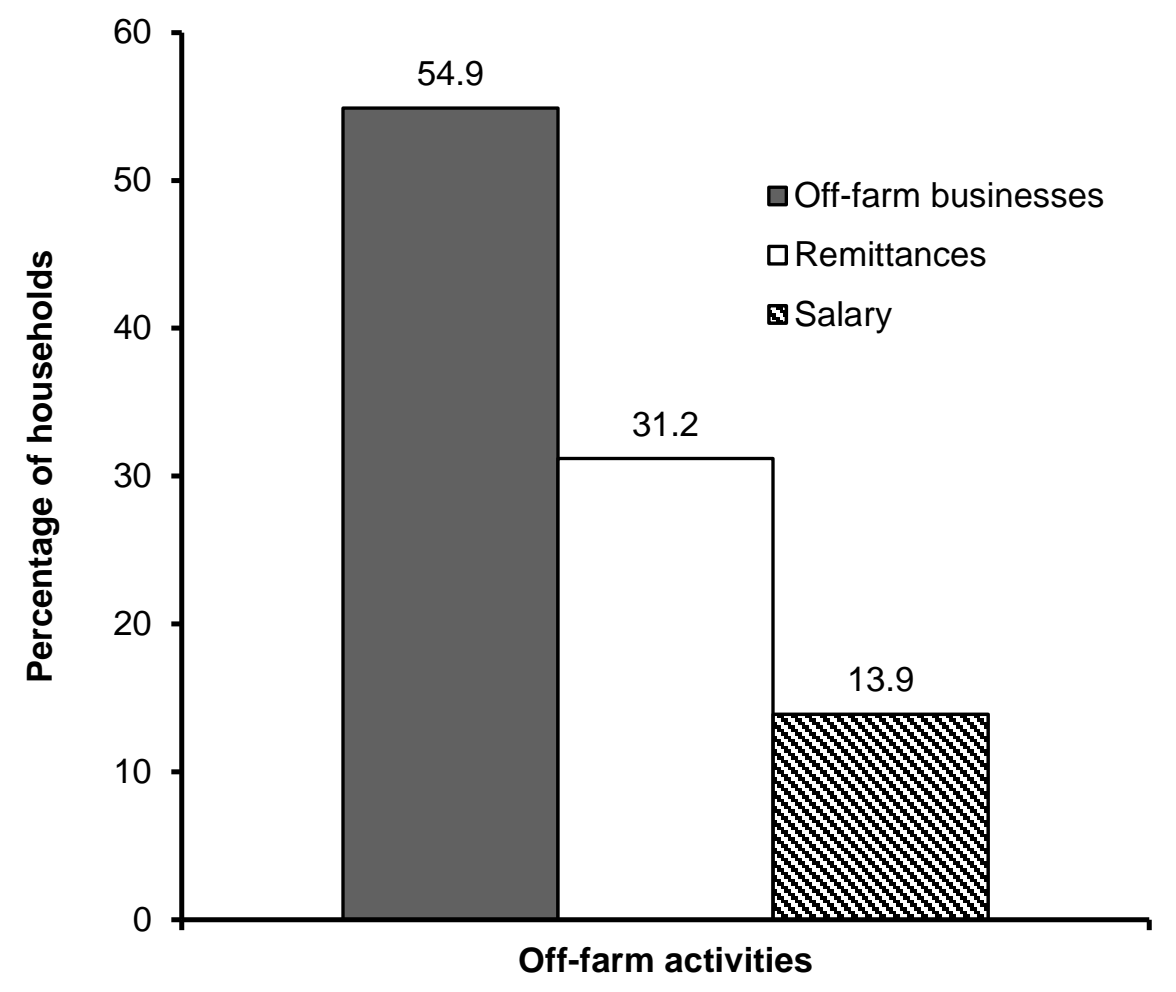

As discussed, agriculture-related outcome variables of interest here are the proportion of coffee sold as shelled green beans and average coffee prices received by farmers. Table 3.2 shows that MM users sell a higher proportion of their harvest as shelled coffee, whereas for coffee prices we do not observe significant differences. 
The lower part of Table 3.2 shows the covariates used in the regression models. For many of these covariates, significant differences between MM users and non-users can be observed. MM users tend to have younger household heads that are more likely to be male, have higher levels of education, more land and other productive assets. MM users also spend more money on agricultural inputs. Finally, MM users have more neighbors that also have a MM account, possibly pointing at social influence in technology adoption at the local level. This neighborhood variable was captured by asking how many of the respondent's 10 nearest neighbors in the village use MM services.

Overall, Table 3.2 reveals that mobile money users and non-users differ in terms of many of the observed characteristics. Against this background it is likely that they will differ in terms of unobserved characteristics, too. Hence, without controlling for confounding factors, the differences in outcome variables cannot be interpreted as effects of mobile money adoption. As explained above, the panel regression models control for observed and unobserved heterogeneity to estimate unbiased treatments effects of mobile money adoption.

\subsubsection{Determinants of Mobile Money Adoption}

Table 3.3 presents the estimation results from the binary choice model to explain MM adoption, as described in equation (3.1). In column (1), we used an RE probit estimator. The estimation results show that larger households are more likely to be MM users. Obviously, when there are more household members the probability that at least one of them uses MM services increases. Male-headed households are significantly less likely to use MM, which is striking because the descriptive statistics above suggested otherwise. The reason for this discrepancy is that females tend to be disadvantaged in terms of other factors, such as education and asset ownership. The model results, which control for such other factors, suggest that females may possibly benefit 
more from MM services than males. This is plausible given that female farmers are often more time-constrained, so that innovations that help reduce the costs of market and financial transactions are particularly welcome.

Table 3.3: Determinants of mobile money adoption

\begin{tabular}{|c|c|c|c|c|c|}
\hline & (1) Probit, RE & (2) LP, RE & (3) LP, FE & (4) LP, RE & (5) LP, FE \\
\hline \multirow{2}{*}{$\begin{array}{l}\text { Education of household } \\
\text { head (years) }\end{array}$} & 0.035 & 0.001 & 0.001 & 0.001 & 0.001 \\
\hline & $(0.079)$ & $(0.003)$ & $(0.007)$ & $(0.003)$ & $(0.007)$ \\
\hline \multirow{2}{*}{$\begin{array}{l}\text { Age of household head } \\
\text { (years) }\end{array}$} & -0.021 & -0.001 & 0.001 & -0.001 & 0.001 \\
\hline & $(0.018)$ & $(0.001)$ & $(0.002)$ & $(0.001)$ & $(0.002)$ \\
\hline \multirow[t]{2}{*}{ Male head (dummy) } & $-1.228 * *$ & -0.023 & & -0.022 & \\
\hline & $(0.562)$ & $(0.024)$ & & $(0.023)$ & \\
\hline \multirow{2}{*}{$\begin{array}{l}\text { Household size } \\
\text { (persons) }\end{array}$} & $0.272 * * *$ & $0.009 * * *$ & $0.014 * *$ & $0.008 * *$ & $0.014 * *$ \\
\hline & $(0.090)$ & $(0.003)$ & $(0.007)$ & $(0.003)$ & $(0.007)$ \\
\hline \multirow[t]{2}{*}{ Land owned (ha) } & 0.148 & 0.007 & & 0.007 & \\
\hline & $(0.273)$ & $(0.010)$ & & $(0.010)$ & \\
\hline \multirow[t]{2}{*}{ Square of land owned } & -0.088 & 0.001 & & 0.001 & \\
\hline & $(0.084)$ & $(0.003)$ & & $(0.003)$ & \\
\hline \multirow{2}{*}{$\begin{array}{l}\text { Productive assets } \\
\text { (UGX) }\end{array}$} & $1.1 \mathrm{E}-07$ & $9.0 \mathrm{E}-09$ & $-9.7 \mathrm{E}-09$ & $6.7 \mathrm{E}-09$ & $-9.7 \mathrm{E}-09$ \\
\hline & $(1.5 \mathrm{E}-07)$ & $(6.3 \mathrm{E}-09)$ & $(1.2 \mathrm{E}-08)$ & $(6.3 \mathrm{E}-09)$ & $(1.2 \mathrm{E}-08)$ \\
\hline \multirow{2}{*}{$\begin{array}{l}\text { Distance to tarmac road } \\
(\mathrm{km})\end{array}$} & 0.012 & 0.001 & & 0.001 & \\
\hline & $(0.026)$ & $(0.001)$ & & $(0.001)$ & \\
\hline \multirow{2}{*}{$\begin{array}{l}\text { Masaka district } \\
\text { (dummy) }\end{array}$} & 0.393 & 0.022 & & 0.011 & \\
\hline & $(0.499)$ & $(0.021)$ & & $(0.021)$ & \\
\hline \multirow{2}{*}{$\begin{array}{l}\text { Migrant household } \\
\text { (dummy) }\end{array}$} & 0.551 & 0.008 & -0.024 & 0.009 & -0.024 \\
\hline & $(0.514)$ & $(0.023)$ & $(0.033)$ & $(0.023)$ & $(0.031)$ \\
\hline \multirow{2}{*}{$\begin{array}{l}\text { MM service center in } \\
\text { village (dummy) }\end{array}$} & $6.544 * * *$ & $0.574 * * *$ & $0.557 * * *$ & $0.570 * * *$ & $0.558 * * *$ \\
\hline & $(0.819)$ & $(0.027)$ & $(0.044)$ & $(0.027)$ & $(0.044)$ \\
\hline \multirow{2}{*}{$\begin{array}{l}\text { Neighbors using MM } \\
\text { (number) }\end{array}$} & $1.510 * * *$ & $0.051 * * *$ & $0.043 * * *$ & $0.050 * * *$ & $0.043 * * *$ \\
\hline & $(0.197)$ & $(0.004)$ & $(0.007)$ & $(0.004)$ & $(0.007)$ \\
\hline \multirow[t]{2}{*}{ Year 2015} & $0.443 * * *$ & $0.017 * * *$ & $0.023 * * *$ & $0.015 * *$ & $0.023 * * *$ \\
\hline & $(0.144)$ & $(0.006)$ & $(0.008)$ & $(0.006)$ & $(0.008)$ \\
\hline \multirow{2}{*}{$\begin{array}{l}\text { Mobile phone use } \\
\text { (dummy) }\end{array}$} & & & & $0.080 * * *$ & 0.006 \\
\hline & & & & $(0.03)$ & $(0.040)$ \\
\hline \multirow[t]{2}{*}{ Constant } & $-897.932 * * *$ & $-34.237 * *$ & $-47.013 * * *$ & $-29.710 * *$ & $-46.317 * * *$ \\
\hline & $(290.840)$ & $(12.642)$ & (15.699) & $(12.723)$ & $(16.331)$ \\
\hline No. of observations & 874 & 874 & 874 & 874 & 874 \\
\hline No. of households & 480 & 480 & 480 & 480 & 480 \\
\hline Wald $\chi^{2}$ & $123.45 * * *$ & $2388.20 * * *$ & & $2419.07 * * *$ & \\
\hline$F$-value & & & $133.93 * * *$ & & $118.75^{* * *}$ \\
\hline Hausman test $\chi 2$ & & & $12.20 *$ & & $16.32 * *$ \\
\hline
\end{tabular}

Notes: Estimation coefficients are shown with standard errors in parentheses. LP, linear probability model; RE, random effects; FE, fixed effects; MM, mobile money; ${ }^{* * *} \mathrm{p}<0.01, * * \mathrm{p}<0.05,{ }^{*} \mathrm{p}<0.1$

Access to MM service centers and neighborhood effects also affect MM adoption in a positive way. Having a MM service center in the village means that cash withdrawals and deposits are not associated with much travel time, which can be an important incentive for adoption. Service 
centers can also provide technical support. Similarly, having more neighbors using the same technology facilitates access to information and technical advice through social networks. Social network effects were also shown to be relevant for the adoption of other innovations (Matuschke and Qaim, 2009; Andersson et al., 2015). Finally, the 2015 year dummy has a highly significant positive coefficient, underlining the rapidly increasing adoption of MM technology over time.

There is no straightforward FE estimator for the probit model, which is why we used a linear probability (LP) specification to explain adoption in columns (2) and (3) of Table 3.3. For the LP model, estimation coefficients can directly be interpreted as marginal effects. In the LP models, access to MM service centers and neighborhood effects remain positive and significant. The Hausman test statistic, which is shown at the bottom of Table 3.3, confirms that the FE model is preferred. The FE model results in column (3) show that having a service center in the village increases the probability of $\mathrm{MM}$ adoption by 56 percentage points. This is a large effect, underlining the importance of a wide service center network for wide and rapid MM adoption.

In columns (4) and (5) of Table 3.3, we additionally included the adoption of mobile phones as an explanatory variable. This was not included previously, because use of mobile phones is potentially endogenous. In the RE specification in column (4), mobile phone adoption influences MM adoption in a positive way, even though the effect is relatively small. The small effect may be due to the widespread use of mobile phones in the study region. In the FE specification in column (5), the effect of mobile phone adoption turns insignificant. The effects of other variables are hardly affected by including mobile phone use.

It is worth mentioning that education, farm size, other productive assets, and distance to roads are all insignificant in the models in Table 3.3, regardless of the exact specification. Hence, factors related to human capital, wealth, and road infrastructure, which often affects the adoption of other 
types of technologies, seems to be less relevant for MM adoption. The results suggest that MM could positively affect the lives of even those people that are often more disadvantaged in terms of other innovations.

\subsubsection{Impact of Mobile Money Use on Household Welfare}

Table 3.4 shows the estimation results of the models that we use to evaluate the impact of MM on household welfare, as described in equation (3.2). The RE specifications in columns (1) and (3) of Table 3.4 suggest that MM use affects household income and per capita consumption in a positive and significant way.

\section{Table 3.4: Impact of mobile money use on household welfare}

\begin{tabular}{|c|c|c|c|c|}
\hline & \multicolumn{2}{|c|}{$\begin{array}{c}\text { Household income } \\
\text { (thousand UGX per year) }\end{array}$} & \multicolumn{2}{|c|}{$\begin{array}{l}\text { Per capita consumption } \\
\text { (UGX per day) }\end{array}$} \\
\hline & (1) RE & (2) FE & (3) RE & (4) FE \\
\hline Mobile money use (dummy) & $\begin{array}{l}493.2 * * \\
(223.2)\end{array}$ & $\begin{array}{c}394.3 \\
(367.8)\end{array}$ & $\begin{array}{l}261.3 * * \\
(112.6)\end{array}$ & $\begin{array}{c}48.2 \\
(194.3)\end{array}$ \\
\hline Education of head (years) & $\begin{array}{c}107.7 * * * \\
(34.6)\end{array}$ & $\begin{array}{c}-1.5 \\
(78.8)\end{array}$ & $\begin{array}{l}37.1 * * \\
(17.3)\end{array}$ & $\begin{array}{c}42.8 \\
(41.6)\end{array}$ \\
\hline Age of head (years) & $\begin{array}{c}-18.8^{* *} \\
(7.6)\end{array}$ & $\begin{array}{l}-30.7 \\
(24.2)\end{array}$ & $\begin{array}{c}11.0 * * * \\
(3.8)\end{array}$ & $\begin{array}{l}-11.8 \\
(12.8)\end{array}$ \\
\hline Male head (dummy) & $\begin{array}{l}212.8 \\
(255.4)\end{array}$ & & $\begin{array}{c}165.3 \\
(127.2)\end{array}$ & \\
\hline Household size (persons) & $\begin{array}{l}-56.8 \\
(35.5)\end{array}$ & $\begin{array}{l}-82.1 \\
(75.0)\end{array}$ & $\begin{array}{c}-304.1 * * * \\
(17.8)\end{array}$ & $\begin{array}{c}-276.3 * * * \\
(39.6)\end{array}$ \\
\hline Land owned (ha) & $\begin{array}{l}418.6^{* * * *} \\
(84.9)\end{array}$ & & $\begin{array}{l}87.3 * * \\
(42.9)\end{array}$ & \\
\hline Productive assets (UGX) & $\begin{array}{c}7.3 \mathrm{E}-04 * * * \\
(6.9 \mathrm{E}-05)\end{array}$ & $\begin{array}{c}5.7 \mathrm{E}-04 * * * \\
(1.3 \mathrm{E}-04)\end{array}$ & $\begin{array}{c}2.9 \mathrm{E}-04 * * * \\
(3.5 \mathrm{E}-05)\end{array}$ & $\begin{array}{c}2.6 \mathrm{E}-04 * * * \\
(6.6 \mathrm{E}-05)\end{array}$ \\
\hline Distance to tarmac road $(\mathrm{km})$ & $\begin{array}{c}6.9 \\
(11.1)\end{array}$ & & $\begin{array}{l}7.8 \\
(5.5)\end{array}$ & \\
\hline Masaka district (dummy) & $\begin{array}{l}777.1 * * * \\
(225.5)\end{array}$ & & $\begin{array}{c}-62.0 \\
(111.9)\end{array}$ & \\
\hline Year 2015 & $\begin{array}{r}35.4 \\
(65.5\end{array}$ & $\begin{array}{c}89.6 \\
(80.0)\end{array}$ & $\begin{array}{c}142.2 * * * \\
(33.8)\end{array}$ & $\begin{array}{c}192.7 * * * \\
(42.3)\end{array}$ \\
\hline Constant & $\begin{array}{l}-7.4 \mathrm{E} 04 \\
(1.3 \mathrm{E} 05)\end{array}$ & $\begin{array}{l}-1.8 \mathrm{E} 05 \\
(1.6 \mathrm{E} 05)\end{array}$ & $\begin{array}{c}-1.8 \mathrm{E} 05^{* * *} \\
(6.8 \mathrm{E} 04)\end{array}$ & $\begin{array}{l}-2.8 \mathrm{E} 05 * * * \\
(8.4 \mathrm{E} 04)\end{array}$ \\
\hline No. of observations & 874 & 874 & 874 & 874 \\
\hline No. of households & 480 & 480 & 480 & 480 \\
\hline Wald $\chi^{2}$ & $380.1 * * *$ & & $408.49 * * *$ & \\
\hline$F$-value & & $5.28 * * *$ & & $19.8^{* * *}$ \\
\hline Hausman test $\chi^{2}$ & & 6.42 & & 5.65 \\
\hline
\end{tabular}

Notes: Estimation coefficients are shown with standard errors in parentheses. RE, random effects; FE, fixed effects; $* * * \mathrm{p}<0.01, * * \mathrm{p}<0.05, * \mathrm{p}<0.1 ; 1$ US\$ $=2,690$ UGX 
While the FE specifications in columns (2) and (4) also show positive treatment effects, these are not statistically significant. However, the Hausman test statistics, which are shown at the bottom of Table 3.4, are not significant, so the RE models are preferred because of their higher estimation efficiency. The results confirm earlier work that showed positive welfare effects of MM use on rural households in Kenya and Uganda (Kikulwe et al., 2014; Munyegera and Matsumoto, 2016).

As we use linear model specifications, the coefficient estimates in Table 3.4 can also be interpreted as marginal effects. Controlling for other factors, MM use has increased annual household income by 493 thousand UGX on average. Compared to mean income levels of nonadopters, this is equivalent to an increase of $19 \%$. The MM treatment effect on daily per capita consumption is $261 \mathrm{UGX}$, equivalent to a $7.5 \%$ increase over mean consumption levels of nonadopters.

Other covariates that affect household income and per capita consumption in a positive way are education, land owned, and other productive assets. Households located in Masaka have higher incomes than those located in Luwero District.

\subsubsection{Impact of Mobile Money Use on Remittances and Off-Farm Income}

Table 3.5 shows the estimation results for impacts on remittances and off-farm income. Columns (1) and (2) show RE and FE specifications with remittances received as dependent variable. In both models, MM use has a positive coefficient, but this is not statistically significant. This does not necessarily prove that MM has no impact on remittances. But the insignificant results suggest that - even if there were a positive effect on remittances received - this is probably not the main or the only pathway through which MM affects household welfare. We therefore look at other possible pathways in the following. 
In columns (3) and (4) of Table 3.5, the effects of MM use on total off-farm income, including remittances, are shown. Given the significant Hausman test statistic, the FE model in column (4) is preferred. MM use has increased annual off-farm income by 322 thousand UGX, equivalent to a $44 \%$ treatment effect over non-adopters. In columns (5) and (6) of Table 3.5, the same models are shown but now using off-farm income without remittances included as dependent variable.

Table 3.5: Impact of mobile money use on remittances and off-farm income

\begin{tabular}{|c|c|c|c|c|c|c|}
\hline & \multicolumn{2}{|c|}{ Remittances } & \multicolumn{2}{|c|}{$\begin{array}{l}\text { Off-farm income including } \\
\text { remittances }\end{array}$} & \multicolumn{2}{|c|}{$\begin{array}{l}\text { Off-farm income without } \\
\text { remittances }\end{array}$} \\
\hline & (1) RE & (2) FE & (3) RE & (4) FE & (5) RE & (6) FE \\
\hline \multirow{2}{*}{$\begin{array}{l}\text { Mobile money use } \\
\text { (dummy) }\end{array}$} & 44.2 & 22.5 & $257.5 * *$ & $322.4^{*}$ & $229.2 * *$ & $302.6^{*}$ \\
\hline & $(28.9)$ & $(54.9)$ & (112.9) & $(184.9)$ & (109.6) & $(175.0)$ \\
\hline \multirow{2}{*}{$\begin{array}{l}\text { Education of head } \\
\text { (years) }\end{array}$} & 3.2 & -15.8 & $69.1 * * *$ & -7.2 & $66.8 * * *$ & 10.4 \\
\hline & $(4.4)$ & (11.7) & $(17.6)$ & $(39.6)$ & $(17.21)$ & $(37.5)$ \\
\hline \multirow[t]{2}{*}{ Age of head (years) } & $4.8 * * *$ & 1.0 & -4.2 & -4.7 & $-8.8 * *$ & -5.6 \\
\hline & $(1.0)$ & $(3.6)$ & $(3.9)$ & $(12.2)$ & $(3.8)$ & $(11.5)$ \\
\hline \multirow[t]{2}{*}{ Male head (dummy) } & -39.2 & & 167.9 & & $233.8 *$ & \\
\hline & (31.9) & & (129.8) & & $(127.7)$ & \\
\hline Household size & 5.6 & -2.7 & -3.5 & 15.1 & -4.9 & 18.9 \\
\hline (persons) & $(4.4)$ & $(11.3)$ & $(18.0)$ & $(38.0)$ & $(17.6)$ & $(36.0)$ \\
\hline \multirow[t]{2}{*}{ Land owned (ha) } & $21.5 * *$ & & -27.7 & & -36.7 & \\
\hline & $(10.4)$ & & (42.9) & & (41.6) & \\
\hline Productive assets & & & $2.1 \mathrm{E}-04 * * *$ & $1.7 \mathrm{E}-04 * * *$ & $1.8 \mathrm{E}-04 * * *$ & $1.5 \mathrm{E}-04 * *$ \\
\hline$(\mathrm{UGX})$ & & & $(3.5 \mathrm{E}-05)$ & $(6.3 \mathrm{E}-05)$ & $(3.4 \mathrm{E}-05)$ & $(6.0 \mathrm{E}-05)$ \\
\hline Distance to tarmac road & $-3.8 * * *$ & & -8.3 & & -4.7 & \\
\hline$(\mathrm{km})$ & $(1.4)$ & & $(5.6)$ & & $(5.5)$ & \\
\hline Migrant household & 17.8 & 3.4 & 62.9 & 266.4 & 59.5 & 263.6 \\
\hline (dummy) & $(32.9)$ & $(52.7)$ & $(124.5)$ & $(177.3)$ & (120.1) & $(167.8)$ \\
\hline Masaka district & 28.1 & & -170.8 & & -185.1 & \\
\hline (dummy) & $(27.4)$ & & $(114.7)$ & & $(113.3)$ & \\
\hline \multirow[t]{2}{*}{ Year 2015} & $32.5 * * *$ & $40.6 * * *$ & 37.9 & $73.4 *$ & 6.6 & 33.7 \\
\hline & (9.3) & (12.0) & $(33.1)$ & $(40.5)$ & $(31.4)$ & $(38.4)$ \\
\hline \multirow[t]{2}{*}{ Constant } & $-6.5 \mathrm{E} 05 * * *$ & $-8.1 \mathrm{E} 05 * * *$ & $-7.6 \mathrm{E} 05$ & $-1.5 \mathrm{E} 06^{*}$ & $-1.4 \mathrm{E} 05$ & $-6.8 \mathrm{E} 05$ \\
\hline & $(1.8 \mathrm{E} 05)$ & $(2.4 \mathrm{E} 05)$ & $(6.6 \mathrm{E} 05)$ & $(8.1 \mathrm{E} 05)$ & $(6.3 \mathrm{E} 05)$ & (7.7E05) \\
\hline No. of observations & 874 & 874 & 874 & 874 & 874 & 874 \\
\hline No. of households & 480 & 480 & 480 & 480 & 480 & 480 \\
\hline Wald $\chi^{2}$ & $72.52 * * *$ & & $127.3 * * *$ & & $114.99 * * *$ & \\
\hline$F$-value & & $4.36 * * *$ & & $4.06 * * *$ & & $2.76 * * *$ \\
\hline Hausman test $\chi^{2}$ & & 5.88 & & $26.6^{* * * *}$ & & $22.7 * * *$ \\
\hline
\end{tabular}

Notes: All dependent variables are measured in thousand UGX per year. Estimation coefficients are shown with standard errors in parentheses. RE, random effects; FE, fixed effects; *** $\mathrm{p}<0.01, * * \mathrm{p}<0.05, * \mathrm{p}<0.1 ; 1 \mathrm{US} \$=2,690$ UGX

Again, the FE specification is preferred. The treatment effect remains large and positive (303 UGX, equivalent to $47 \%$ over non-adopters), which also confirms that remittances are not the 
main pathway of the MM effect on off-farm income. As explained, small-scale businesses in trade, transportation, and handicrafts may particularly benefit from the new savings and money transfer opportunities through MM technology.

Other covariates that positively affect off-farm income are education, male household heads, and productive assets. These effects are more pronounced in the RE specifications, which is due to the low data variation over time within households for these variables.

\subsubsection{Impact of Mobile Money Use on Agricultural Marketing}

Table 3.6 shows the model estimates with the proportion of coffee sold as shelled green beans as dependent variable. As explained, the proportion of shelled beans is used as a proxy for selling in higher-value markets rather than selling immediately after harvest in unprocessed form. Given the insignificant Hausman test statistic, we prefer the RE model that is shown in column (1) of Table 3.6. Our hypothesis that MM has a positive effect on the proportion of coffee sold as shelled beans is confirmed. The treatment effect of 0.12 implies that the proportion is increased by 12 percentage points. Given that non-adopters of MM sold about $23 \%$ of their coffee as shelled beans, the 12 percentage point increase is a substantial effect.

Other covariates that also affect the proportion of coffee sold as shelled beans are productive assets and input use per hectare, meaning that wealthier farmers and those with access to better production technology also find it easier to sell in high-value markets. Farmers in Masaka also sell a larger proportion of their coffee in shelled form. On the other hand, distance to road reduces the proportion of coffee sold as shelled beans. The significantly negative year dummy coefficient is due to the fact that rainfalls and coffee yields were lower in 2015 than in 2012 (UBOS, 2015). In these models, we also control for a few other farm characteristics that may affect coffee output 
and marketing decisions, such as age of coffee plants and time needed to reach the coffee plots.

These other variables are not statistically significant.

\section{Table 3.6: Impact of mobile money use on proportion of coffee sold as shelled beans}

\begin{tabular}{|c|c|c|}
\hline & (1) RE & (2) FE \\
\hline \multirow[t]{2}{*}{ Mobile money use (dummy) } & $0.116^{* *}$ & $0.186^{* *}$ \\
\hline & $(0.051)$ & $(0.085)$ \\
\hline \multirow[t]{2}{*}{ Education of head (years) } & -0.002 & 7.5E-05 \\
\hline & $(0.008)$ & $(0.018)$ \\
\hline \multirow[t]{2}{*}{ Age of head (years) } & 0.003 & 0.002 \\
\hline & $(0.002)$ & $(0.006)$ \\
\hline \multirow[t]{2}{*}{ Male head (dummy) } & 0.043 & \\
\hline & $(0.057)$ & \\
\hline \multirow{2}{*}{ Household size (persons) } & -0.006 & 0.008 \\
\hline & $(0.008)$ & $(0.018)$ \\
\hline \multirow[t]{2}{*}{ Land owned (ha) } & 0.010 & \\
\hline & $(0.019)$ & \\
\hline \multirow[t]{2}{*}{ Productive assets (UGX) } & $2.9 \mathrm{E}-08^{*}$ & $-2.5 \mathrm{E}-08$ \\
\hline & (1.6E-08) & (2.9E-08) \\
\hline \multirow[t]{2}{*}{ Distance to tarmac road $(\mathrm{km})$} & $-0.009 * * *$ & -0.007 \\
\hline & $(0.002)$ & $(0.006)$ \\
\hline \multirow[t]{2}{*}{ Age of productive coffee trees (years) } & 0.005 & -0.006 \\
\hline & $(0.022)$ & $(0.037)$ \\
\hline \multirow[t]{2}{*}{ Square of age of productive coffee trees } & $-3.3 \mathrm{E}-05$ & 0.001 \\
\hline & $(0.001)$ & $(0.001)$ \\
\hline \multirow[t]{2}{*}{ Input use (UGX per ha) } & $1.6 \mathrm{E}-06^{* *}$ & 8.7E-07 \\
\hline & $(7.0 \mathrm{E}-07)$ & $(1.0 \mathrm{E}-06)$ \\
\hline \multirow[t]{2}{*}{ Walking time to coffee plots (minutes) } & -0.008 & \\
\hline & $(0.006)$ & \\
\hline \multirow[t]{2}{*}{ Masaka district (dummy) } & $0.447 * * *$ & \\
\hline & $(0.051)$ & \\
\hline \multirow[t]{2}{*}{ Year 2015} & $-0.059 * * *$ & $-0.066 * * *$ \\
\hline & $(0.016)$ & $(0.020)$ \\
\hline \multirow[t]{2}{*}{ Constant } & $118.696^{* *}$ & $132.208 * * *$ \\
\hline & $(31.928)$ & $(39.244)$ \\
\hline No. of observations & 874 & 874 \\
\hline No. of households & 480 & 480 \\
\hline Wald $\chi^{2}$ & $161.50 * * *$ & \\
\hline$F$-value & & $2.69 * * *$ \\
\hline Hausman test $\chi 2$ & & 9.02 \\
\hline
\end{tabular}

Notes: Estimation coefficients are shown with standard errors in parentheses. RE, random effects; FE, fixed effects; $* * * \mathrm{p}<0.01, * * \mathrm{p}<0.05, * \mathrm{p}<0.1$

Effects of MM use on coffee prices received are shown in Table 3.7. Given the insignificant Hausman test statistic, we concentrate on the RE model in column (1). MM use has a positive effect. Controlling for other factors, MM adopters have received 231 UGX more per $\mathrm{kg}$ of shelled coffee (or weight equivalence of coffee sold in other forms), which translates into a 5\% increase 
over the mean price received by non-adopters. The higher price can be explained by MM users selling more of their coffee as shelled beans and having better access to buyers in higher-value markets.

\section{Table 3.7: Impact of mobile money use on coffee prices received}

\begin{tabular}{|c|c|c|}
\hline Model & (1) RE & (2) FE \\
\hline Mobile money use (dummy) & $\begin{array}{c}230.5^{* *} \\
(115.8)\end{array}$ & $\begin{array}{l}318.1^{*} \\
(179.5)\end{array}$ \\
\hline Education of head (years) & $\begin{array}{c}-2.4 \\
(18.3)\end{array}$ & $\begin{array}{l}-20.1 \\
(38.4)\end{array}$ \\
\hline Age of head (years) & $\begin{array}{c}10.5^{* * * *} \\
(4.0)\end{array}$ & $\begin{array}{c}13.2 \\
(11.8)\end{array}$ \\
\hline Male head (dummy) & $\begin{array}{c}80.6 \\
(136.0)\end{array}$ & \\
\hline Household size (persons) & $\begin{array}{l}-20.2 \\
(18.7)\end{array}$ & $\begin{array}{c}-8.7 \\
(36.6)\end{array}$ \\
\hline Land owned (ha) & $\begin{array}{l}77.0^{*} \\
(43.9)\end{array}$ & \\
\hline Productive assets (UGX) & $\begin{array}{c}1.1 \mathrm{E}-04 * * * \\
(3.6 \mathrm{E}-05)\end{array}$ & $\begin{array}{l}1.1 \mathrm{E}-04 * \\
(6.1 \mathrm{E}-05)\end{array}$ \\
\hline Distance to tarmac road $(\mathrm{km})$ & $\begin{array}{c}-24.9 * * * \\
(5.8)\end{array}$ & \\
\hline Masaka district (dummy) & $\begin{array}{c}1,687 * * * \\
(121.1)\end{array}$ & \\
\hline Year 2015 & $\begin{array}{c}-195.4 * * * \\
(32.5)\end{array}$ & $\begin{array}{c}-212.5^{* * * *} \\
(39.1)\end{array}$ \\
\hline Constant & $\begin{array}{c}3.9 \mathrm{E} 06 * * * \\
(6.5 \mathrm{E} 05)\end{array}$ & $\begin{array}{c}4.3 \mathrm{E} 06 * * * \\
(7.8 \mathrm{E} 05)\end{array}$ \\
\hline No. of observations & 874 & 874 \\
\hline No. of households & 480 & 480 \\
\hline Wald $\chi^{2}$ & $317.54 * * *$ & \\
\hline$F$-value & & $6.19^{* * *}$ \\
\hline Hausman test $\chi 2$ & & 1.82 \\
\hline
\end{tabular}

Notes: Estimation coefficients are shown with standard errors in parentheses. RE, random effects; FE, fixed effects; $* * * \mathrm{p}<0.01, * * \mathrm{p}<0.05, * \mathrm{p}<0.1 ; 1$ US $\$=2,690$ UGX

Other covariates that influence the coffee price received includes the farmer's age, farm size, productive assets, and distance to road (Table 3.7). Productive assets include vehicles and transport equipment, so the positive effect is unsurprising. Longer distances to the tarmac road lead to higher transportation costs, thus lowering prices for agricultural outputs sold at the farm gate. The positive effect for Masaka is due to better developed market infrastructure in that district. Finally, prices in 2015 were significantly lower than in 2012. This reflects international price developments. Also, due to lower rainfalls the average coffee quality was lower in 2015 . 


\subsubsection{Robustness Checks}

The model estimates above revealed positive impacts of MM use on household welfare. Better access to higher-value agricultural markets and off-farm income sources were identified as important impact pathways. In this subsection, we discuss additional tests that were conducted to check for the robustness of these results.

Estimation of unbiased treatment effects with observational data is often plagued by unobserved heterogeneity between treated and untreated subjects, in our case MM adopting and non-adopting households. As explained, this can lead to endogeneity bias. We have used FE panel estimators to test and control for time-invariant unobserved heterogeneity. However, FE panel models cannot control for time-variant unobserved heterogeneity, which may occur, for instance, when MM adopters also adopt other innovations more rapidly. If the adoption of such other innovations is beneficial, positive correlation with MM adoption could result in overestimated treatment effects. To test for such bias, we re-estimated all impact models by additionally including proxies for the adoption of other technical and institutional innovations.

A first proxy that we employ is the use of mobile phones, which is known to improve market access and household welfare through various channels (Nakasone et al., 2014). A second proxy we use is coffee certification. Many farmers in our sample are certified under sustainability labels such as UTZ, Fairtrade, or organic. ${ }^{5}$ Recent studies showed that participation in certification schemes can affect sales prices and welfare of smallholder farm households (Weber, 2011; Chiputwa et al., 2015; Chiputwa and Qaim, 2016; van Rijsbergen et al., 2016). We measure certification in terms of a simple dummy, which is time-variant. During the three years in-

\footnotetext{
${ }^{5}$ Certification in this context does not imply that farmers would sell all of their coffee only in certified channels. Certification is a necessary condition to sell in certain channels, but farmers still decide themselves where, to whom, and in what form they sell their coffee.
} 
between our two survey rounds newly certified households and dropouts from certification schemes were both observed in the sample. Results from the additional model estimates are shown in Table 3.8 (upper and medium part). The treatment effects are not much affected by inclusion of these innovation proxies. MM effects remain positive, significant, and similar in magnitude to the estimates discussed above. We conclude that the findings are robust to timevariant unobserved heterogeneity at the individual farm and household level.

Table 3.8: Robustness checks of treatment effects with additional covariates

\begin{tabular}{|c|c|c|c|c|c|c|c|}
\hline & Income & $\begin{array}{l}\text { Per capita } \\
\text { consumpti } \\
\text { on }\end{array}$ & $\begin{array}{c}\text { Remittanc } \\
\text { es }\end{array}$ & $\begin{array}{l}\text { Off-farm } \\
\text { income } \\
\text { (incl. } \\
\text { remit.) }\end{array}$ & $\begin{array}{l}\text { Off-farm } \\
\text { income } \\
\text { (w/o } \\
\text { remit.) }\end{array}$ & $\begin{array}{l}\text { Prop. of } \\
\text { coffee } \\
\text { sold as } \\
\text { shelled } \\
\text { beans }\end{array}$ & $\begin{array}{l}\text { Coffee } \\
\text { price }\end{array}$ \\
\hline \multicolumn{8}{|c|}{ Effects of including mobile phone use } \\
\hline MM use & $503.45^{* *}$ & $241.3 * *$ & 34.8 & $327.4^{*}$ & $303.3 *$ & $0.12 * *$ & $235.3 * *$ \\
\hline (dummy) & (226.8) & $(114.5)$ & $(29.6)$ & $(185.2)$ & $(175.4)$ & $(0.05)$ & $(117.3)$ \\
\hline \multirow[t]{2}{*}{ MP use (dummy) } & -74.7 & 138.5 & 56.0 & 127.4 & 18.2 & -0.01 & -39.2 \\
\hline & $(290.4)$ & $(147.3)$ & $(38.3)$ & (216.4) & (204.9) & $(0.07)$ & $(148.9)$ \\
\hline Model & RE & RE & RE & FE & FE & RE & RE \\
\hline \multicolumn{8}{|c|}{ Effects of including certification } \\
\hline MM use & $492.7 * *$ & $244.8 * *$ & 40.1 & $325.1^{*}$ & $306.3 *$ & $0.09 *$ & $298.8^{*}$ \\
\hline (dummy) & (223.9) & (112.6) & $(29.0)$ & $(185.1)$ & $(175.1)$ & $(0.05)$ & $(179.9)$ \\
\hline Certified & 7.5 & $231.0^{* *}$ & 44.3 & -157.7 & -214.8 & $0.33 * * *$ & 288.0 \\
\hline (dummy) & $(225.0)$ & $(112.0)$ & $(28.3)$ & (289.6) & $(274.0)$ & $(0.05)$ & $(281.6)$ \\
\hline Model & RE & RE & RE & FE & FE & RE & FE \\
\hline \multicolumn{8}{|c|}{ Effects of including MM service center in village } \\
\hline MM use & -299.9 & 32.8 & 71.1 & -104.5 & -146.7 & 0.11 & $549.9 * *$ \\
\hline (dummy) & $(341.4)$ & $(173.1)$ & $(45.1)$ & (264.6) & $(250.0)$ & $(0.08)$ & $(-348.1)$ \\
\hline MM service & $1060.0 * * *$ & $305.1^{*}$ & -35.7 & $600.6^{* *}$ & $632.1 * *$ & 0.01 & -348.1 \\
\hline center (dummy) & (346.4) & (175.7) & $(45.8)$ & (267.6) & $(252.8)$ & $(0.08)$ & $(263.7)$ \\
\hline Model & RE & $\mathrm{RE}$ & $\mathrm{RE}$ & FE & FE & $\mathrm{RE}$ & FE \\
\hline
\end{tabular}

Notes: Estimation coefficients are shown with standard errors in parentheses. Dependent variables are defined and measured as in Tables 3.4 to 3.7. In addition to the variables shown, covariates as used in Tables 3.4 to 3.7 were included for estimation. Hausman tests were used to decide for each model whether the random effects or fixed effects model is more appropriate. RE, random effects; FE, fixed effects; MM, mobile money; MP, mobile phone; $* * * \mathrm{p}<0.01, * * \mathrm{p}<0.05, * \mathrm{p}<0.1$

Beyond the individual farm and household level, unobserved heterogeneity at the regional level may possibly play a role. For instance, it could be that MM adopters are more prevalent in regions with a more vibrant local economy. While time-invariant differences in economic 
conditions are controlled for in the FE models, time-variant unobserved differences in the local economy are not.

One variable that may possibly capture time-variant economic activities at the village level is the existence of a MM service center. We used this variable to explain MM adoption, but we did not include it in the impact models above. Since the MM service centers only offer services related to mobile phone-based technologies, the existence of a service center in the village is unlikely to affect the outcome variables directly through pathways other than MM or mobile phone adoption. Yet it is possible that MM service centers are established especially in villages with strong economic growth. Hence, the time-variant village-level dummy 'MM service center' could perhaps be used as a proxy for the vitality of the local economy. We tried to use this proxy as an additional covariate with results being summarized in the lower part of Table 3.8. Inclusion of MM service center makes the models very instable. Most of the treatment effects become insignificant; several of them even turn negative. The problem is that the service center variable is highly correlated with MM adoption ( $r=0.81$ ), thus causing serious issues of multicollinearity.

One could argue that MM service center may not be a neutral proxy for the local economy anyway, because the use of mobile technologies is not only a symptom but actually an important trigger of new economic activities in rural Africa (Aker and Mbiti, 2010; Jack and Suri, 2014; Kikulwe et al., 2014; Munyegera and Matsumoto, 2016). Unfortunately, we have no other proxy for the vitality of the local economy. However, we argue that time-variant regional conditions are unlikely to cause significant bias in our case, because the sample was taken from three coffeegrowing locations in Central Uganda, which are all similar in terms of natural and socioeconomic conditions. During the three years in-between the two survey rounds, changes in the local 
economy occurred through more farmers using MM. We have not noticed other major changes in terms of new infrastructure investments or institutional shifts in any of the three locations.

\section{Table 3.9: Robustness checks testing for reverse causality}

\begin{tabular}{|c|c|c|c|}
\hline & $\begin{array}{l}\text { (1) Household income, } \\
\text { IV }\end{array}$ & $\begin{array}{l}\text { (2) MM adoption } \\
\text { LP, RE }\end{array}$ & $\begin{array}{l}\text { (3) MM adoption, } \\
\text { LP, FE }\end{array}$ \\
\hline Mobile money use (dummy) & $\begin{array}{c}917.7 * * * \\
(330.0)\end{array}$ & & \\
\hline Education of head (years) & $\begin{array}{c}108.4 * * * \\
(33.4)\end{array}$ & $\begin{array}{c}0.002 \\
(0.003)\end{array}$ & $\begin{array}{c}0.001 \\
(0.007)\end{array}$ \\
\hline Age of head (years) & $\begin{array}{c}-16.4^{* *} \\
(7.3)\end{array}$ & $\begin{array}{l}-0.001^{*} \\
(0.001)\end{array}$ & $\begin{array}{c}0.001 \\
(0.002)\end{array}$ \\
\hline Male head (dummy) & $\begin{array}{c}162.9 \\
(243.0)\end{array}$ & $\begin{array}{l}-0.023 \\
(0.024)\end{array}$ & \\
\hline Household size (persons) & $\begin{array}{l}-65.0^{*} \\
(34.8)\end{array}$ & $\begin{array}{c}0.009 * * * \\
(0.003)\end{array}$ & $\begin{array}{l}0.013 * \\
(0.007)\end{array}$ \\
\hline Land owned (ha) & $\begin{array}{c}428.9 * * * \\
(84.8)\end{array}$ & $\begin{array}{c}0.011 \\
(0.008)\end{array}$ & \\
\hline Productive assets (UGX) & $\begin{array}{l}\text { 7.2E-04**** } \\
(6.8 \mathrm{E}-05)\end{array}$ & $\begin{array}{l}1.2 \mathrm{E}-08^{*} \\
(6.7 \mathrm{E}-09)\end{array}$ & $\begin{array}{l}-4.4 \mathrm{E}-09 \\
(1.2 \mathrm{E}-08)\end{array}$ \\
\hline Distance to tarmac road $(\mathrm{km})$ & $\begin{array}{c}6.4 \\
(10.7)\end{array}$ & $\begin{array}{c}0.001 \\
(0.001)\end{array}$ & \\
\hline Masaka district (dummy) & $\begin{array}{c}772.6 * * * \\
(212.2)\end{array}$ & $\begin{array}{c}0.025 \\
(0.021)\end{array}$ & \\
\hline Migrant household (dummy) & & $\begin{array}{c}0.008 \\
(0.023)\end{array}$ & $\begin{array}{l}-0.021 \\
(0.032)\end{array}$ \\
\hline $\begin{array}{l}\text { MM service center in village } \\
\text { (dummy) }\end{array}$ & & $\begin{array}{c}0.576 * * * \\
(0.027)\end{array}$ & $\begin{array}{c}0.566 * * * \\
(0.044)\end{array}$ \\
\hline Neighbors using MM (number) & & $\begin{array}{c}0.051 * * * \\
(0.004)\end{array}$ & $\begin{array}{c}0.043 * * * \\
(0.007)\end{array}$ \\
\hline Year 2015 & $\begin{array}{l}-28.3 \\
(77.4)\end{array}$ & $\begin{array}{c}0.017 * * \\
(0.006)\end{array}$ & $\begin{array}{c}0.023 * * \\
(0.008)\end{array}$ \\
\hline Income (thousand UGX) & & $\begin{array}{l}-4.1 \mathrm{E}-06 \\
(3.2 \mathrm{E}-06)\end{array}$ & $\begin{array}{c}-9.7 \mathrm{E}-06 * * \\
(4.7 \mathrm{E}-06)\end{array}$ \\
\hline Constant & $\begin{array}{c}54,184.5 \\
(155,770.9) \\
\end{array}$ & $\begin{array}{c}-34.654 * * * \\
(12.540) \\
\end{array}$ & $\begin{array}{c}-46.620 * * * \\
(15.636) \\
\end{array}$ \\
\hline No. of observations & 874 & 874 & 874 \\
\hline No. of households & 480 & 480 & 480 \\
\hline$F$-value & $43.30 * * *$ & & $120.51 * * *$ \\
\hline Wald $\chi 2$ & & $2394.19 * * *$ & \\
\hline
\end{tabular}

Notes: Estimation coefficients are shown with standard errors in parentheses. In column (1), mobile money use was instrumented with the number of neighbors using MM (out of 10 nearest neighbors). IV, instrumental variable model; LP, linear probability model; RE, random effects; FE, fixed effects; MM, mobile money; *** $\mathrm{p}<0.01$, ** $\mathrm{p}<0.05, * \mathrm{p}<0.1$

Another way of testing for endogeneity bias is through using an instrumental variable (IV) estimator. This also includes endogeneity bias due to possible issues of reverse causality. The IV estimator requires an instrument that is correlated with MM adoption but has no direct effect on 
the outcome variables of interest. Since we use multiple outcome variables, finding suitable instruments for all of them proved difficult. But we were able to identify one instrument that worked quite well in the household income model, namely the number of neighbors using MM (out of the 10 nearest neighbors).

As explained above, this variable is a proxy for access to MM information and technical advice through informal social networks. Results of the IV income model are shown in column (1) of Table 3.9. The estimated treatment effect is positive and significant, confirming that MM adoption has a positive effect on household income, also after accounting for reverse causality and other possible sources of endogeneity that the FE estimators may not have fully controlled for. Interestingly, the magnitude of the MM effect in Table 3.9 is even bigger than the RE and FE estimates in Table 3.4, suggesting that the treatments effects discussed above are rather conservative estimates.

Indeed, it is possible that reverse causality may lead to an underestimated income effect of MM adoption. This could occur if poorer households are more likely to adopt. As household income is endogenous itself, we did not include income as an explanatory variable in the adoption models discussed in Table 3.3. However, when included income has a negative coefficient in the adoption model, which is insignificant in the RE specification but turns significant in the FE specification (see columns 2 and 3 of Table 3.9). Unlike many other technologies that are associated with risks or sizeable upfront investments, opening a MM account is cheap and easy, so income constraints do not seem to deter MM adoption. This is consistent with previous research looking at MM adoption in East Africa (Jack et al., 2013; Kikulwe et al., 2014). The FE specification in column (3) of Table 3.9 even suggests that households with negative income 
growth in recent years had stronger incentives to adopt MM, probably hoping to improve their economic situation.

The robustness checks imply that some caution is warranted when interpreting the exact magnitudes of the estimated treatment effects. However, the general finding that MM adoption has caused positive welfare effects for smallholder farm households in Uganda seems to be fairly robust.

\subsection{Conclusions}

Previous studies showed that the rapid spread of mobile money (MM) in Africa can contribute to welfare gains in rural and urban households. One important mechanism that was mentioned in several studies is through higher remittances that MM users receive from relatives and friends. In this article, we have tested the hypothesis that other impact pathways - that were not analyzed previously - can also be important, especially in a smallholder farm context. In particular, we had hypothesized that MM services can help farmers to access higher-value markets and thus receive higher prices for their products. We had also hypothesized that the use of MM can increase offfarm income beyond remittances.

These hypotheses were tested and confirmed with panel data from smallholder coffee farmers in Uganda. Panel regression models revealed that the adoption of MM technology contributes to higher household welfare in terms of income and consumption. Total household income gains through MM were estimated at $19 \%$. Gains in off-farm income were estimated at around $45 \%$, regardless of whether or not remittances were included. In fact, the MM treatment effect on remittances alone was found to be insignificant, suggesting that MM services may be more relevant for other off-farm income sources in this particular case. Small businesses in handicrafts and trade and transport services are the most important off-farm income sources for rural 
households in the sample. These businesses benefit from the new savings and money transfer opportunities through MM technology.

MM users were also found to be more likely to sell coffee in dried and shelled form to buyers in higher-value markets instead of selling to local traders immediately after harvest. Due to higher savings and off-farm incomes, MM users are less cash-constrained, so that the need to sell immediately after harvest is reduced. Moreover, MM services facilitate transactions with buyers from outside the local region, because this often involves agreements where product orders, deliveries, and payments do not occur at the same time and place. Controlling for other factors, MM users fetched 5\% higher average prices for their coffee than farmers who were not using this new technology.

We conclude that MM services can contribute to rural development through various pathways. The rapid spread of MM technology within only a few years is remarkable. By $2015,89 \%$ of the randomly selected households in our sample were using mobile phones, and $62 \%$ had a mobile money account. Adoption models showed that factors related to human capital, wealth, and road infrastructure, which typically constrains the adoption of other new technologies, is less relevant for MM technology. And, after controlling for other covariates, female-headed households were found more likely to use MM than male-headed households. These results suggest that MM services are socially inclusive and can positively affect the lives of even those people that are often disadvantaged in terms of other innovations. It is interesting to note that these are purely private-sector driven developments. The analysis also showed that MM use is strongly influenced by access to a MM service center in the village; hence, expanding the service center network can likely increase MM adoption also in remote rural areas. 
The findings from this study should not be widely generalized, as our sample of small-scale coffee growers in Uganda may not be representative of all small farm settings. We also emphasize that panel data with only two rounds of observations, as used here, have their limitations. For instance, if MM adoption is caused by unobserved time-variant heterogeneity in the local economy, the treatment effects may be overestimated. There may also be possible issues of reverse causality. Panel data with more rounds of observations could help to further improve the identification strategy. Finally, we acknowledge that additional impacts and impact pathways - not analyzed here - may also be important. One interesting aspect would be to analyze the gender implications of MM services in greater detail. More research is needed to confirm the findings and further advance the research direction. 


\section{General Conclusions}

\subsection{Main Research Findings}

Mobile phone (MP) technologies and their applications - such as mobile money transfers - are gaining in importance, connecting farming households to markets through enabling market information, money exchange, and market integration, thus aiding inclusive social and economic welfare improvements. Previous research on MP technologies has largely focused on impacts in terms of market access, input and output prices, and remittances. Possible impacts on social welfare indicators - such as household nutrition or gender equality - have largely been neglected, even though improvements in such social welfare dimensions were prioritized in the United Nation's Sustainable Development Goals (SDGs). A knowledge gap thus existed in understanding household income, agriculture, gender equality, and nutrition effects of MP technologies that are now widely adopted in many developing countries. With regards to key MP technologies' applications like mobile money (MM) services, previous research showed that the rapid spread of MM in Africa could contribute to welfare gains in rural and urban households. One important mechanism that was mentioned in several studies is through higher remittances that $\mathrm{MM}$ users receive from relatives and friends. However linkages of $\mathrm{MM}$ services with supplementary income activities generating off-farm incomes, and access to high-value markets had not yet been investigated.

We addressed these knowledge gaps by studying impacts of MP use on household income, gender equality, and nutrition using regression models and panel data from Uganda. We used simultaneous equations, and gender disaggregated data to study MP use impact pathways. With regards to MM services we tested the hypothesis that other impact pathways - that were not analyzed previously - could also be important, especially in a smallholder farm context. 
Precisely, we hypothesized that MM services could help farmers to access higher-value markets and thus receive higher prices for their products. We also hypothesized that the use of MM could increase off-farm income beyond remittances.

Where we used aggregated MP use as the treatment, results showed that MP use has significant positive impacts on household income, gender equality, and nutrition. From reduced-form panel modes, where we controlled for other factors, mobile phone use increases household income by $26 \%$ and gender equality by $19 \%$. Similarly, mobile phone use enhances household food security and dietary quality. Gender-disaggregated data analysis also reveals that female MP use bears stronger effects on household income, gender equality, food security and dietary quality than male MP use. These improvements are inclusive since are proved at household level both in aggregate terms and gender disaggregated perspectives. More clearly, female MP use improved gender equality or women empowerment through increasing proportions of productive assets owned by females or jointly with their spouses. These income and gender effects of female MP use impacted positively on household nutrition; hence we identify income and gender equality as key MP impact pathways for household nutrition. These are interesting findings since traditions in rural settings of SSA have largely alienated women from household resources' ownership. Such alienation of women from resource ownership had left household sectors dominated by women for instance feeding and health lacking in household resources allocation. Female MP use significantly increased income; through reduced transactions costs and access to market information.

With regards to the hypotheses tested for MM services use, results revealed that the adoption of MM technology contributes to higher household welfare in terms of household income and consumption. Total household income gains through MM were estimated at 19\%. Gains in off- 
farm income were estimated at around $45 \%$, regardless of whether or not remittances were included. In fact, the MM treatment effect on remittances alone was found to be insignificant, suggesting that MM services may be more relevant for other off-farm income sources in this particular case. Small businesses in handicrafts and trade and transport services are the most important off-farm income sources for rural households in the sample. These businesses benefit from the new savings and money transfer opportunities through MM technology. MM users were also found to be more likely to sell coffee in dried and shelled form to buyers in higher-value markets instead of selling to local traders immediately after harvest. Due to higher savings and off-farm incomes, MM users are less cash-constrained, so that the need to sell immediately after harvest is reduced. Moreover, MM services facilitate transactions with buyers from outside the local region, because this often involves agreements where product orders, deliveries, and payments do not occur at the same time and place. Controlling for other factors, MM users fetched 5\% higher average prices for their coffee than farmers who were not using this new technology.

In brief we conclude that MP use and MM services can contribute to rural social and economic development through various pathways. The rapid spread of MP and MM technologies within only a few years is remarkable and implies their acceptance and importance to adopting households.

On the other hand, although results largely turned out as hypothesized, data were collected from only central Uganda, and used only two survey rounds, hence results may not be widely interpreted. However, we stress that - to the best of our knowledge - this is the first study exploring impacts of MP use on gender equality and nutrition using a panel survey with genderdisaggregated observations. This is also the first study to analyze impacts of MM services on off- 
farm incomes and agricultural marketing. Of course, there is always scope for further improvements in terms of the geographical coverage and the data and methodologies used.

\subsection{Policy and Research Recommendations}

In terms of policy recommendations, we would suggest that policies on MP technological dissemination be targeted to all gender equally. However, while aiming at improvements in sectors of welfare where women are of particular importance, for instance health and nutrition, policies targeted more specifically to women could be even more beneficial. Policy makers should also create awareness to households about other MM impact pathways like off-farm employment, and high-value agricultural markets' access where MM services can even have better welfare impacts, compared to the popular remittances' access. If policies help households to exploit these other pathways that are seemingly more sustainable than remittances, then sustainable reduction in rural household poverty and hunger would be more possible. MM services, were also almost exclusively supplied by private companies, hence tax holidays that encourage private investment would help cement provision of MM services.

In terms of future research recommendations, using panel data with more rounds of observations could help to further improve the identification strategy. Finally, we acknowledge that additional impacts and impact pathways - not analyzed here - may also be important. One interesting aspect would be to analyze household micronutrient consumption and gender implications of $\mathrm{MM}$ services in greater detail. More research is also needed to confirm the findings and further advance the research direction. 


\section{References}

Aker, J.C., 2010. Information from markets near and far: mobile phones and agricultural markets in Niger. American Economic Journal: Applied Economics 2(3), 46 - 59

Aker, J.C., 2011. Dial "A" for agriculture: a review of information and communication technologies for agricultural extension in developing countries. Agricultural Economics $42,631-647$

Aker, J.C., Ksoll, C., 2016. Can mobile phones improve agricultural outcomes? Evidence from a randomized experiment in Niger. Food Policy 60, $44-51$

Aker, J.C., Ksoll, C., Lybbert, T. J., 2012. Can Mobile Phones Improve Learning? Evidence from a Field Experiment in Niger., American Economic Journal: Applied Economics, 4(4), 94 $-120$

Aker, J.C., Mbiti, I.M., 2010. Mobile phones and economic development in Africa. Journal of Economic Perspectives, 24(3), $207-232$

Alsop, R., Bertelsen, F.M., Holland, J., 2006. Empowerment in Practice: From Analysis to Implementation. The World Bank, Washington, DC.

Andersson, C.I.M., Chege, C.G.K., Rao, E.J.O., Qaim, M., 2015. Following up on smallholder farmers and supermarkets in Kenya. American Journal of Agricultural Economics, 97, $1247-1266$

Arimond, M. 2004. Dietary diversity is associated with child nutritional status: evidence from 11 demographic and health surveys. Journal of Nutrition, 134 (10), $2579-2585$

Bayes, A., 2001. Infrastructure and rural development: Insights from Grameen Bank village phone initiative in Bangladesh. Agricultural Economics, 25, 261 - 272 
Bhavnani, A., Won-Wai Chiu, R., Janakiram, S., Silarszky, P., 2008. The role of mobile phones in sustainable rural poverty reduction. World Bank; Washington, DC

Blandon, J., Henson, S., Islam, T., 2009. Marketing preferences of small-scale farmers in the context of new agrifood systems: a stated choice model. Agribusiness 25, $251-267$

Blattman, C., Jensen, R., Roman, R., 2002. Assessing the Need and Potential of Community Networking for Development in Rural India Special Issue: ICTs and Community Networking. The Information Society, 9 (5), 349 - 364

Blauw, S., Franses, H.P., 2016. Off the hook: measuring the impact of mobile telephone use on economic development of households in Uganda using copulas. Journal of Development Studies, 52, $315-330$

Boulos, K. N. M., Wheeler, S., Tavares, C., Jones, R., 2011. How smartphones are changing the face of mobile and participatory healthcare: an overview, with example from eCAALYX. BioMedical Engineering OnLine 10 (24), 1 - 14

Burgess, R., Pande, R., Wong, G., 2005. Banking for the Poor: Evidence from India. Journal of the European Economic Association, 3 (2-3), 268 - 278

Cameron, A. C., Trivedi, K. P., 2005. Microeconometrics: Methods and Applications. Cambridge University Press, UK.

Castells, M., Fernandez-Ardevol, M., Qiu, J. L., Sey, A., 2004. The mobile communication society: A cross-cultural analysis of available evidence on the social uses of wireless communication technology, Research report prepared for the International Workshop on Wireless Communication Policies and Prospects: A Global Perspective, held at the Annenberg School for Communication, University of Southern California, Los Angeles, 
October 8th and 9th 2004. From: http://www.itu.dk/ ldn/Lars/MCS.pdf (December 19, 2014)

Chiputwa, B., Qaim, M., 2016. Sustainability standards, gender, and nutrition among smallholder farmers in Uganda. Journal of Development Studies 52, 1241 - 1257

Chiputwa, B., Spielman, D. J., Qaim, M., 2015. Food standards, certification, and poverty among coffee farmers in Uganda. World Development, 66, 400 - 412

Chowdhury, S., Negassa, A., Torero, M., 2005. Market Institutions: Enhancing the Value of Rural-Urban Links. FCND Discussion Paper 195; IFPRI, Washington DC

Donner, J., 2007. The use of mobile phones by microentrepreneurs in Kigali, Rwanda: Changes to social and business networks. Information Technologies and International Development $3(2), 3-19$

Doss, C.R., Deere, C.D., Oduro, A.D., Swaminathan, H., 2014. The gender asset and wealth gaps. Development 57 (3-4), $400-409$

Duncombe, R., Boateng, R., 2009. Mobile phones and financial services in developing countries: A review of concepts, methods, issues, evidence and future research directions. Third World Quarterly 30 (7), 1237 - 1258

Fafchamps, M., Hill, R.V., 2005. Selling at the farm gate or traveling to market. American Journal of Agricultural Economics, 87, 717 - 734

Fischer, E., Qaim, M., 2012. Gender, agricultural commercialization, and collective action in Kenya. Food Security 4 (3), $441-453$

Fozdar, I. B., Kumar, S. L., 2007. Mobile learning and student retention. International Review of Research in Open and Distance Learning 8 (2), 1-18 
GSMA., 2014. GSMA Intelligence; Mobile Money for the Unbanked: https://gsmaintelligence.com/topics/3363/dashboard/ Accessed 7. Dec. 2014

Hoddinott, J., 2012. Agriculture, health, and nutrition: Towards conceptualizing the linkages. In: Fan, S., Pandya-Lorch, R., Eds. Reshaping Agriculture for Nutrition and Health. International Food Policy Research Institute, Washington, DC, pp. 13 - 20

Hoddinott, J., Rosegrant, M., Torero, M., 2013. Investments to reduce hunger and under nutrition. Global Problems, Smart Solutions ed. by B. Lomborg (Cambridge, Cambridge University Press), UK

Jack, W., Ray, A., Suri, T., 2013. Transaction networks: evidence from mobile money in Kenya. The American Economic Review, 103, 356 - 361

Jack, W., Suri, T., 2014. Risk sharing and transactions costs: evidence from Kenya's mobile money revolution. The American Economic Review, 104, 183 - 223

Jensen, T.R., 2007. The digital provide: information (technology), market performance, and welfare in the South Indian fisheries sector. Quarterly Journal of Economics, 122, 879924.

Jensen, T. R., 2010. Information, Efficiency and Welfare in Agricultural Markets. International Association of Agricultural Economists, pp. 204 - 216

Jones, A. D., Shrinivas, A., Bezner-Kerr, R., 2014. Farm production diversity is associated with greater household dietary diversity in Malawi: Findings from nationally representative data. Food Policy, 46, $1-12$

Jussawalla, M (1999): The impact of ICT convergence on development in the Asian region. Telecommunications Policy, 23, $217-234$ 
Kabunga, N. S., Dubois, T., Qaim, M., 2014. Impact of tissue culture banana technology on farm household income and food security in Kenya. Food Policy, 45, 25 - 34

Kennedy, G., Ballard, T., Dop, M.C., 2011. Guidelines for Measuring Household and Individual Dietary Diversity. Food and Agriculture Organization, Rome

Kikulwe, E. M., Fischer, E., Qaim, M., 2014. Mobile money, smallholder farmers, and household welfare in Kenya. PLOS ONE 9(10), e109804.

Kirui, O.K., Okello, J.J., Nyikal, R.A., Njiraini, G.W., 2013. Impact of mobile phone-based money transfer services in agriculture: evidence from Kenya. Quarterly Journal of International Agriculture 52(2), $141-162$

Koppmair, S., Kassie, M., M, Qaim., 2017. Farm production, market access and dietary diversity in Malawi. Public Health Nutrition, 20 (2), 325 - 335

Levine, R., 2005. Finance and Growth: Theory and Evidence. Handbook of Economic Growth, 1 (A), $865-934$

Malmodin, J., Moberg, A., Lunden, D., Finnveden, G., Lövehagen, N., 2010. Greenhouse gas emissions and operational electricity use in the ICT and entertainment \& media sectors. Journal of Industrial Ecology, 14(5), $770-79$

Matuschke, I., Qaim, M., 2009. The impact of social networks on hybrid seed adoption in India. Agricultural Economics, 40, $493-505$

Masset, E., Haddad, L., Cornelius, A., Isaza-Castro, J., 2012. Effectiveness of agricultural interventions that aim to improve nutritional status of children: Systematic review. British Medical Journal 344, doi. 10.1136/bmj.d8222. 
Mittal, S., Gandhi, S., Tripathi, G., 2010. Socio-Economic Impact of Mobile Phones on Indian Agriculture, A working paper 246, Indian Council for Research on International Economic Relations, India

Mundlak, Y., 1978. On the pooling of time series and cross section data. Econometrica, 46 (1), 69 $-85$

Munyegera, G.K., Matsumoto, T., 2016. Mobile money, remittances, and household welfare: panel evidence from rural Uganda. World Development, 79, 127 - 137

Murendo, C., Wollni, M., 2016. Mobile money and household food security in Uganda. GlobalFood Discussion Paper 76, University of Goettingen, Germany.

Muto, M., 2012. The Impacts of Mobile Phones and Personal Networks on Rural-to-Urban Migration: Evidence from Uganda. Journal of African Economies 21 (5), 787 - 807

Muto, M., Yamano, T., 2009. The impact of mobile phone coverage expansion on market participation: panel data evidence from Uganda. World Development, 37, 1887 - 1896

Nakasone, E., Torero, M., Minten, B., 2014. The power of information: the ICT revolution in agricultural development. Annual Review of Resource Economics, 6, 533 - 550

Nakasone, E., Torero, M., 2016. A text message away: ICTs as a tool to improve food security. Agricultural Economics, 47, 49-59

Nath, V., 2001. Empowerment and governance through information and communications technologies: Women's perspective. International Information \& Library Review 33, 317 $-339$

Oseni, G., Winters, P., 2009. Rural nonfarm activities and agricultural crop production in Nigeria. Agricultural Economics, 40, 189 - 201 
PRC, 2015. Cell Phones in Africa: Communication Lifeline. Pew Research Center, Washington, DC.

Quisumbing, A. R., Maluccio, J. A., 2003. Resources at marriage and intrahousehold allocation: Evidence from Bangladesh, Ethiopia, Indonesia, and South Africa. Oxford Bulletin of Economics and Statistics, 65, $283-327$

Reardon, T., Timmer, C.P., 2012. The economics of the food system revolution. Annual Review of Resource Economics, 4, 225 - 264

Ruel, M.T., 2003. Operationalizing dietary diversity: A review of measurement issues and research priorities. Journal of Nutrition, 133: 3911S - 3926S

Scott, N., Batchelor, S., Ridley, J., Jorgensen, B., 2004. The impact of mobile phones in Africa, prepared for the Commission for Africa, London, Serneels, P.

Sekabira, H., Bonabana, W. J., Asingwire, N., 2012. Determinants for adoption of information and communications technology (ICT)-based market information services by smallholder farmers and traders in Mayuge District, Uganda. Journal of Development and Agricultural Economics, 4(14), $404-415$

Sekabira, H., Qaim, M., 2017. Mobile money, agricultural marketing, and off-farm income in Uganda. Agricultural Economics, in press.

Sibhatu, K. T., Krishna, V. V., Qaim, M., 2015. Production diversity and dietary diversity in smallholder farm households. Proceedings of the National Academy of Sciences USA $112,10657-10662$

Shiferaw, B., Kassie, M., Jaleta, M., Yirga, C., 2014. Adoption of improved wheat varieties and impacts on household food security in Ethiopia. Food Policy 44, 272 - 284 
Suri, T., Jack, W., Stoker, T.M., 2012. Documenting the birth of a financial economy. Proceedings of the National Academy of Science of the United States of America, 109, $10257-10262$

Tadesse, G., Bahiigwa, G., 2015. Mobile phones and farmers' marketing decisions in Ethiopia. World Development, 68, 296 - 307

Torero, M., Chowdhury, K S., Galdo, V., 2002. Willingness to Pay for the Rural Telephone Service in Bangladesh and Peru. Center for Development Research (ZEF), Bonn

Torero, M., von Braun, J., Eds. 2005. Information and Communication Technologies for Development and Poverty Reduction: The Potential of Telecommunication. Baltimore, MD: The Johns Hopkins University Press for IFPRI.

UBOS., 2013. Statistical Abstract. UBOS, Kampala - Uganda

UBOS., 2015. Statistical Abstract. Uganda Bureau of Statistics, Kampala.

UCC., 2013. Post, Broadcasting \& Telecommunications Annual Market Review 2012/2013. UCC, Kampala - Uganda

UCC., 2015. Postal, Broadcasting and Telecommunications Annual Market and Industry Report. Uganda Communications Commission, Kampala.

Udry, C., 1996. Gender, agricultural production, and the theory of the household. Journal of Political Economy 104 (5), 1010 - 1046

Umeh, G, N., 2008. Assessment of Mass Media Usage for Farm Information by Farmers in AFIKPO LGA of Ebonyi State: implication for information and communication Technology (ICT) Application to Agricultural Technology Transfer. Continental Journal of Information Technology, 2, 9-12 
UN., 2015. Transforming Our World: The 2030 Agenda For Sustainable Development. United Nations - New York, USA

UNCTAD., 2012.: Mobile Money for Business Development in the East African Community: A Comparative Study of Existing Platforms and Regulations. United Nations Publication UNCTAD/DTL/STICT/2012/2 - Switzerland

van Rijsbergen, B., Elbers, W., Ruben, R., Njuguna, S. N., 2016. The ambivalent impact of coffee certification on farmers' welfare: a matched panel approach for cooperatives in Central Kenya. World Development, 77, 277 -292

Von Braun, J., 2010. ICT for the Poor at Large Scale: Innovative Connections to Markets and Services. IFPRI, Washington DC

Weber, G.J., 2011. How much more do growers receive for Fair Trade-organic coffee? Food Policy, 36, 678-685

West, D, M., 2012. How Mobile Technology is Driving Global Entrepreneurship, Pp: 1-10. The Brookings Institution Washington, DC

World Bank., 2014. Migration and Remittances: Recent Developments and Outlook; Special Topic: Forced Migration. Migration and Development Brief 23

Zellner, A., Theil, H., 1962. Three-stage least squares: Simultaneous estimation of simultaneous equations. Econometrica. 30 (1), $54-78$ 
General Appendix (Household Survey Questionnaire - Uganda 2015) 


\section{GlobalFood}

Georg-August University Göttingen, Germany

Department of Agricultural Economics and Rural Development

Household Survey Questionnaire

Smallholder participation in certification schemes / adoption of mobile phone technologies
Questionnaire number:

"Dear Respondent! My name is.... I represent a survey team from the University of Goettingen (Germany). We are following up on respondents who were interviewed in 2012. The objective of this study is to learn more about farmer participation in coffee certification schemes and the adoption of mobile phone technologies. Your cooperation in answering the questions is very much appreciated. Please answer all questions as accurately and truthfully as possible. Your responses will be COMFLETELY CONFDENTAL - hat is we assure that your individual responses will not be disclosed to anyone; the answers will be used for research purpose only. The survey would only take about 2.5 hrs. THANK YOU! For this interview, we would like to talk to the two primary decision makers within this $\mathrm{HH}$ (over 18 years, one male and one female). The primary decision makers are referred to as the persons responsible for decision making both in economic and social terms. The interview has two parts. The first part should be answered by the person we interviewed in 2012. The second part should be answered by both the primary and secondary decision maker SEPARATELY.

\begin{tabular}{|c|c|c|c|}
\hline \multirow[t]{2}{*}{ 1.1 Name } & 1.2 Date of interview & 1.3 Time interview started & 1.4 Time interview ended \\
\hline & $/ 2015$ & & \\
\hline
\end{tabular}

\subsection{HH location}

\begin{tabular}{|c|c|c|c|c|}
\hline 2.1 District (1=Luwero 2=Masaka/Bukomansimbi) & 2.2 County & 2.3 Sub county & 2.4 Parish & 2.5 Village \\
\hline
\end{tabular}

\begin{tabular}{|c|c|c|c|}
\hline 2.6 GPS_north & 2.7 GPS:_south & 2.8 GPS_east & 2.9 Altitude \\
\hline
\end{tabular}

3.1 Who is the primary decision maker (HHD) within this HH? Name:

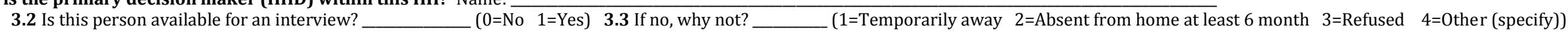

4.1 Who is the secondary decision maker within this $\mathrm{HH}$ (usually spouse)? Name:

4.2 Is this person available for an interview?

(1=Temporarily away $2=$ Absent from home at least 6 month $3=$ Refused $4=0$ ther (specify))

5.0 Respondent interviewed in 2012

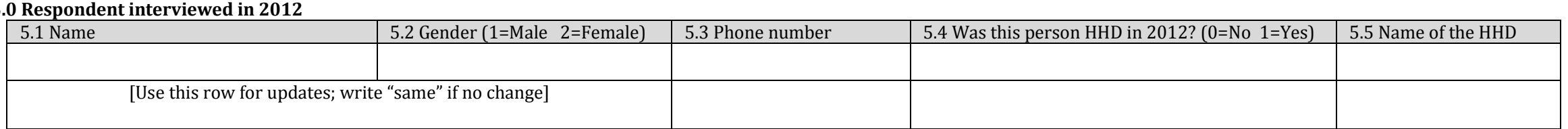

6.1 Who is the first respondent? (Preferably, this should be the respondent interviewed in 2012. This person should answer part 1 and part 2 of the questionnaire)

6.2 Is the respondent of 2012 available to answer part 1 and 2 of the questionnaire? ___ ( $0=$ No $1=Y E S \rightarrow$ make sure information above is correct and update it if necessary; then move to Q7). 6.3 If no, why is the respondent of 2012 not available for an interview? (1=Temporarily away $2=$ Absent from home at least 6 months $3=$ Death $4=0$ ther (specify))

\begin{tabular}{|c|c|c|c|}
\hline 6.4 Name of the first respondent & $\begin{array}{l}6.5 \text { Gender (1=Male } \\
2=\text { Female) }\end{array}$ & 6.6 Phone number & $\begin{array}{l}6.7 \text { This person is the } 1=\text { Primary decision maker } \\
2=\text { Secondary decision maker } 3=\text { =Third decision maker }\end{array}$ \\
\hline \multicolumn{4}{|c|}{0 Who is the second respondent? (This person should answer part 2 of the questionnaire) } \\
\hline 7.1 Name of the second respondent & $\begin{array}{l}7.2 \text { Gender }(1=\text { Male } 2=\mathrm{F} \\
\text { be the same as the first }\end{array}$ & \begin{tabular}{l|l} 
ANNOT & 7.3 Phone number \\
ent! & \\
\end{tabular} & $\begin{array}{l}\text { 7.4 This person is the } 1=\text { Primary decision maker } \\
2=\text { Secondary decision maker } 3=\text { =Third decision maker }\end{array}$ \\
\hline
\end{tabular}




\section{ECTION 1 HOUSEHOLD INFORMATION (E)}

1 How many people normally ${ }^{1}$ ive and eat their meals in this HH (including servants and other workers). Tenants who pay rent are NOT considered members. Number of HH members:

2.0 Please provide the list of names of each member in this HH starting with the head, spouse, and his /her children in order of age.

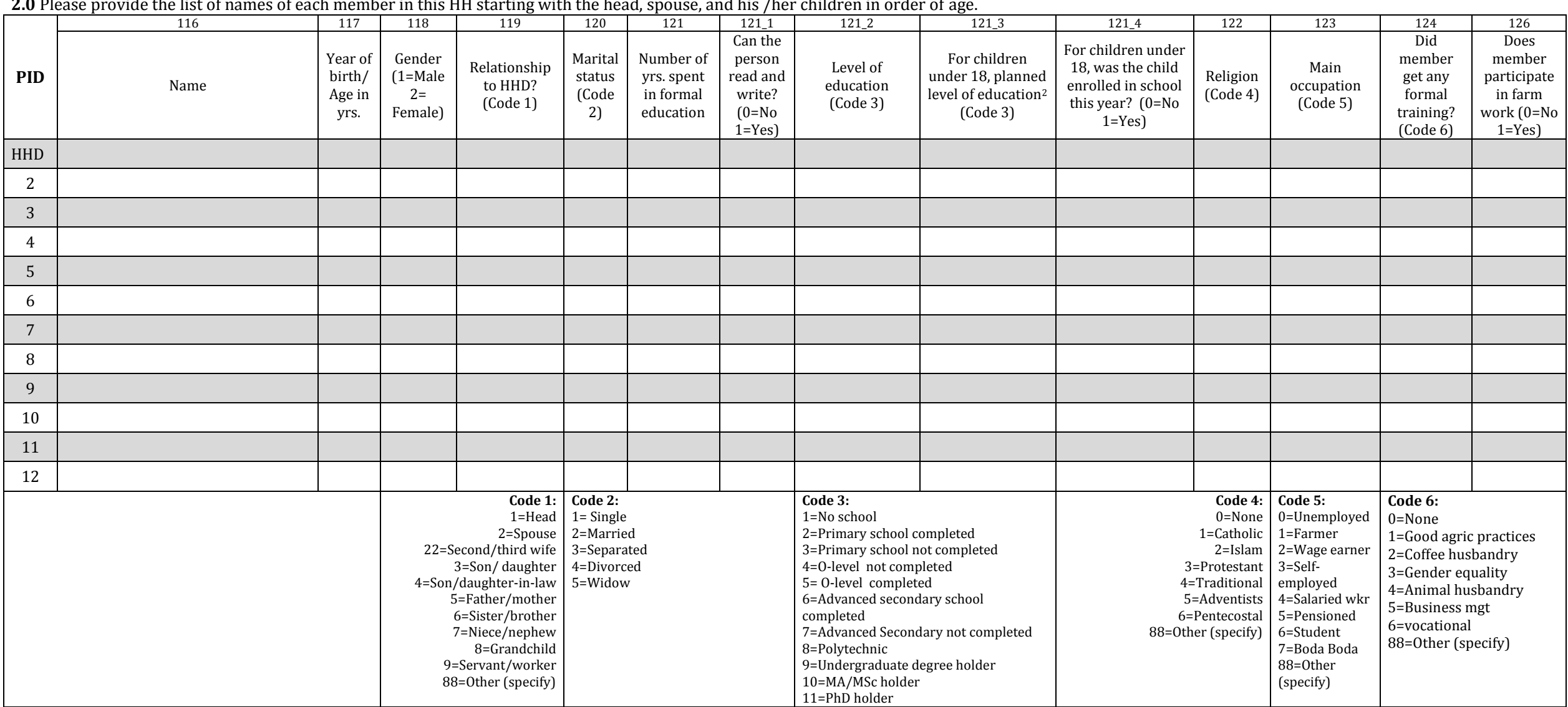

Within the last 6 month, at least 3 days a week

Up to what level of education do the parents plan to educate the child? 
1.1 How long have you been a resident of this community?

1.2 If new resident in this community, in which district were you a resident before settling in this community

1.3 Does this HH have any close relatives (parents, children, uncles, aunties, nieces, in-laws or nephews) leaving in cities away from this village?

1.4 How long have you been involved in farming? years

2.1 Are you a member of any farmer organization (includes association, cooperative, company)

2.2 Since when are you a member?
(indicate year)

$(0=$ No $\rightarrow$ move to $\mathrm{Q} 3 \quad 1=\mathrm{Yes})$

2.3 Who within the HH is a registered member? (1=HHD $2=$ Spouse $3=$ Both $88=0$ ther (specify))

3.1 Are you aware of any of the following certification schemes: Organic, Fairtrade, or UTZ?

3.2 Are you aware of any relative, neighbor or farmer that produces certified coffee?

3.3 If YES, do you interact with any of these persons? ___ $(0=\mathrm{No}, 1=\mathrm{Yes})$

$(0=$ No, $1=$ Yes $)$

4 Is or was this HH ever certified (that is under UTZ, Fairtrade, organic certified) to grow coffee?

5.0 In which of the following certification scheme(s) (did) the HH participate?

\begin{tabular}{|c|l|l|}
\hline & & $\begin{array}{l}0=\text { No, never (move to next scheme) } \\
1=\text { Yes } \\
2=\text { Only formerly }\end{array}$ \\
\hline 1 & UTZ & \\
\hline 2 & Organic & \\
\hline 3 & Fairtrade & \\
\hline 5 & Area certified &
\end{tabular}

$(0=$ No, never $\rightarrow$ move to $Q 4 \quad 1=$ Yes $)$

6.0 Provide information on the total land area owned in the last 12 months.

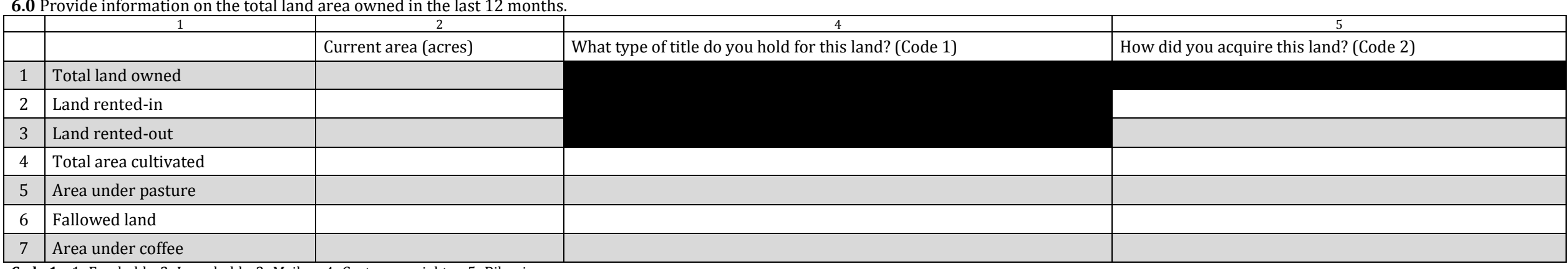

Code 1: 1=Freehold 2=Leasehold 3 =Mailo $\quad 4=$ =ustomary rights $\quad$ =Bibanja

Code 2: $1=$ Purchased $\quad 2=$ Inherited/given by parents/relatives $3=$ =Inherited (spouse) $\quad 4=$ Agreement with land/use rights owner $88=0$ ther (specify)

7.1 On average, how much time (in min) do you spend walking from your homestead to the NEAREST field? (min)

7.2 0 a a

\begin{tabular}{|c|c|c|}
\hline Years certified from & [year] & [year] \\
\hline From & & to \\
\hline From & & to \\
\hline From & & to \\
\hline
\end{tabular}


3.1 What is the average age [years] of your current productive coffee trees ${ }^{1}$ :

3.2 What is the average age [years] of your current unproductive coffee trees ${ }^{1}$ : years.

4 Is coffee production a tradition or a business for you?

5.0 Please provide information on your coffee production and marketing in the last 12 months.

\begin{tabular}{|c|c|c|c|c|c|c|c|c|}
\hline 36 & 1 & 38 & 39 & 40 & 41 & 42 & 43 & 44 \\
\hline Total quantity of red cherries & \multicolumn{4}{|c|}{ Quantity of coffee sold (kg) } & \multicolumn{4}{|c|}{ Coffee price received by farmer } \\
\hline harvested? (bag and kg per bag) & Flowers $^{2}(\mathrm{~kg})$ & $\operatorname{Red}^{3}(\mathrm{~kg})$ & Kiboko $^{4}(\mathrm{~kg})$ & $\operatorname{Kasse}^{5}(\mathrm{~kg})$ & Flowers $^{2}(\mathrm{Shs} / \mathrm{kg})$ & $\operatorname{Red}^{3}(S h s / k g)$ & Kiboko $^{4}(\mathrm{Shs} / \mathrm{kg})$ & $\operatorname{Kasse}^{5}(\mathrm{Shs} / \mathrm{kg})$ \\
\hline _______ bags & & & & & & & & \\
\hline One bag $=\ldots \ldots$ kg & & & & & & & & \\
\hline Total quantity: & $\mathbf{k g}$ & kg & kg & kg & Shs $/ \mathrm{kg}$ & Shs $/ \mathrm{kg}$ & Shs $/ \mathrm{kg}$ & Shs $/ \mathrm{kg}$ \\
\hline
\end{tabular}

6.0 Please provide the following information on coffee marketing:

\begin{tabular}{|c|c|c|c|c|c|c|c|c|c|}
\hline & & 84 & 85 & 86 & 87 & 88 & 89 & 90 & 91 \\
\hline & & \multicolumn{4}{|c|}{ Certified coffee } & \multicolumn{4}{|c|}{ NON-Certified coffee } \\
\hline & & Flower $^{2}$ & Red cherries $^{3}$ & Kiboko ${ }^{4}$ & Kasse $^{5}$ & Flowers $^{2}$ & Red cherries $^{3}$ & Kiboko $^{4}$ & Kasse $^{5}$ \\
\hline 2 & $\begin{array}{l}\text { To whom do you normally sell the following type of coffee? (Allow multiple } \\
\text { responses BUT start with most important)? (Code 1) }\end{array}$ & & & & & & & & \\
\hline 3 & Who in the $\mathrm{HH}$ negotiates for coffee prices with buyers? (Code 2) & & & & & & & & \\
\hline
\end{tabular}

Code 1: 1=Farmer group 2=Middlemen 3=Kibinge FA 4=IBERO LTD 5=KAWACOM 6=Market in Kampala 7=Local exporter 8=Farmer organization/association/company/cooperative 88=0ther (specify)

Code 2: $1=$ HHD $2=$ Spouse $3=$ Jointly $88=0$ ther (specify)

${ }^{1}$ Unproductive coffee is referred to as young coffee trees (gardens where coffee was only planted recently and does not yet produce coffee)

${ }_{3}^{2}$ Flowers: Coffee sold in the field as flowers (also include Green berries)

Red cherries: Wet coffee still in husks sold soon after harvesting

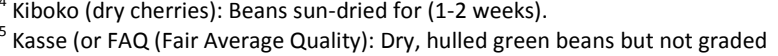


SECTION 4: LAND AREA AND GENERAL CROPS GROWN (A1)

1.0 Please provide the following details on ALL crops grown by the HH in the last 12 months. (Capture details for all the crops grown for the main harvest season and fly-harvest season 1 ).

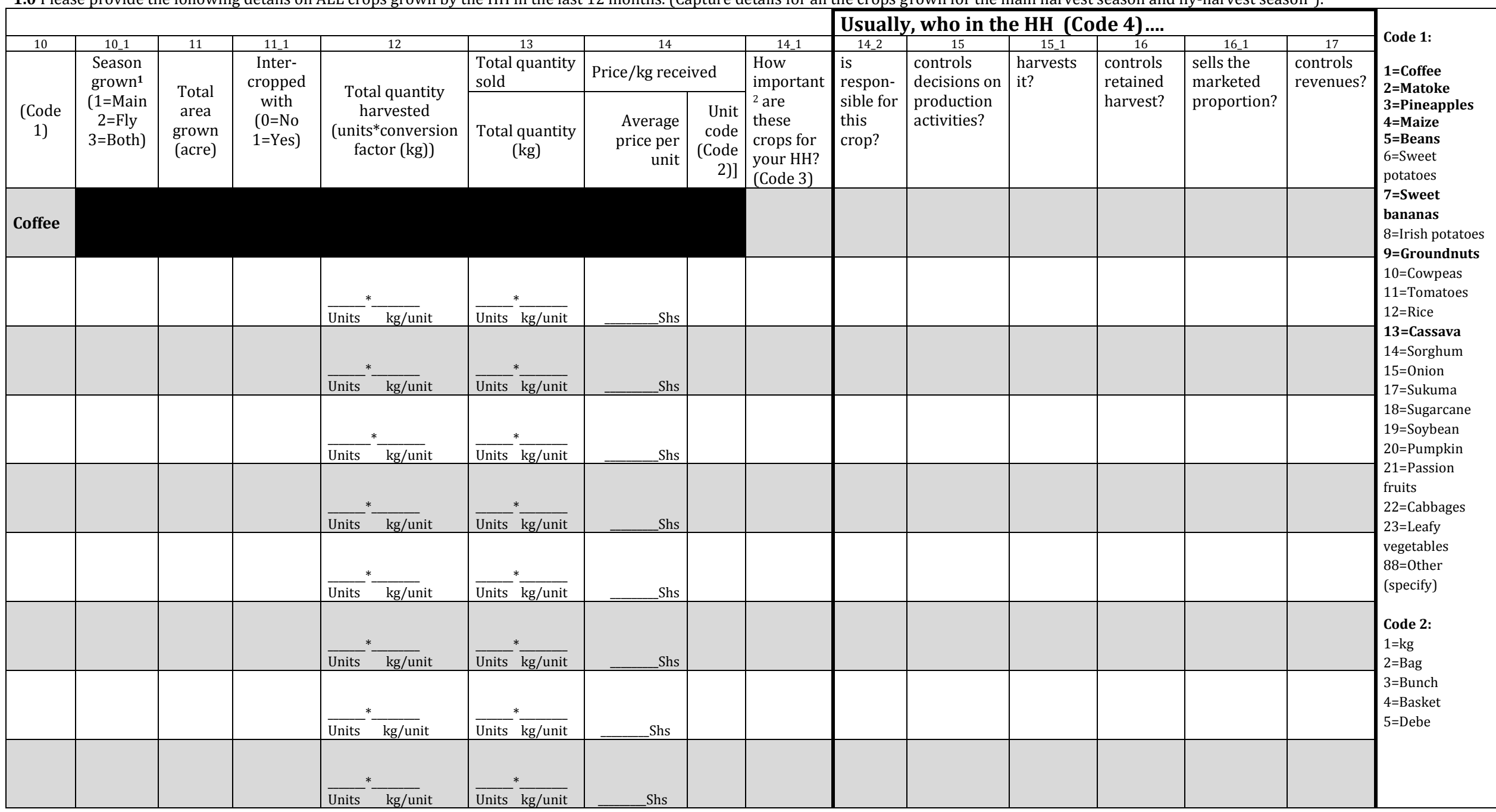

${ }^{1}$ For Masaka district: Main season June-July and Fly season Dec-Jan; for Luwero district: Main season Dec -March and Fly in Aug-Oct Importance in terms of revenues and own consumption 
Code 3: $1=$ Very important $2=$ =mportant $3=$ Not so important Code 4: $1=$ HHD $2=$ Spouse $3=$ Jointly $4=0$ thers (specify)

\section{SECTION 5: PRODUCTION COSTS FOR GENERAL CROPS AND COFFEE (A2/B2)}

1.0 For your cont 12 months (i.e. for the two seasons ${ }^{1}$ ). Then ask for additional costs accuring for other crops individually,

especially seeds and pesticides. Make sure to capture all costs (all categories) if the respective crop is not intercropped with coffee.

\begin{tabular}{|c|c|c|c|c|c|c|c|c|c|c|c|c|c|c|c|c|}
\hline \multirow{4}{*}{\begin{tabular}{|c|}
18 \\
\\
(use \\
Code 1)
\end{tabular}} & \multirow{4}{*}{$\begin{array}{c}18 \_1 \\
\text { Total area } \\
\text { (acre) }\end{array}$} & \multirow{3}{*}{\multicolumn{2}{|c|}{\begin{tabular}{|c|c|}
19 & 20 \\
Seeds/seedlings \\
\end{tabular}}} & \multirow{3}{*}{\multicolumn{2}{|c|}{\begin{tabular}{|c|c|}
46 & 47 \\
Synthetic fertilizer
\end{tabular}}} & \multirow{3}{*}{\multicolumn{3}{|c|}{\begin{tabular}{|c|c|c|}
48 & 49 & $49 \_1$ \\
\multicolumn{3}{|c}{ Manure }
\end{tabular}}} & \multirow{3}{*}{\multicolumn{3}{|c|}{\begin{tabular}{|c|c|c|}
50 & 51 & $51 \_1$ \\
\multicolumn{2}{|c|}{ Pesticides, herbicides, fungicides }
\end{tabular}}} & \multirow{4}{*}{$\begin{array}{c}52 \\
\text { Hired } \\
\text { labor } \\
\text { Total } \\
\text { cost } \\
\text { (Shs) }\end{array}$} & \multirow{4}{*}{$\begin{array}{c}\text { 52_1 } \\
\text { Tarpaulins } \\
\text { Total cost } \\
\text { (Shs) }\end{array}$} & \multirow{4}{*}{$\begin{array}{c}53 \\
\text { Machiner } \\
\text { y total } \\
\text { cost } \\
(\mathrm{Shs})^{2}\end{array}$} & \multirow{4}{*}{$\begin{array}{c}54 \\
\text { Other } \\
\text { costs }^{3} \\
\text { (Shs) }\end{array}$} & \multirow{11}{*}{ 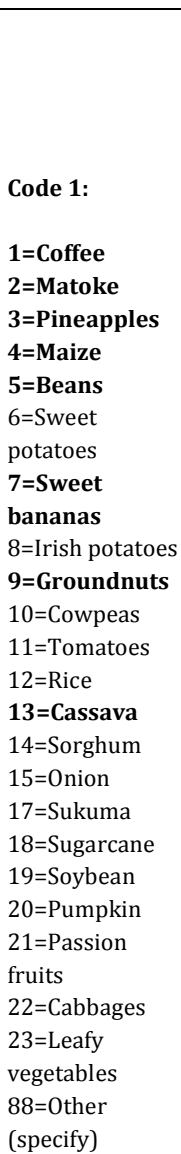 } \\
\hline & & & & & & & & & & & & & & & & \\
\hline & & & & & & & & & & & & & & & & \\
\hline & & $\begin{array}{c}\text { Total } \\
\text { quantity } \\
\text { (kg or unit) }\end{array}$ & $\begin{array}{c}\text { Cost } \\
\text { (Shs/kg or } \\
\text { unit) } \\
\end{array}$ & $\begin{array}{l}\text { Total } \\
\text { quantity } \\
(\mathrm{kg})\end{array}$ & $\begin{array}{l}\text { Cost } \\
\text { (Shs/ } \\
\text { kg) }\end{array}$ & $\begin{array}{c}\text { Quantity } \\
\text { (kg) }\end{array}$ & $\begin{array}{c}\text { Cost } \\
\text { (Shs } / \mathrm{kg})\end{array}$ & $\begin{array}{l}\text { Type } \\
\text { (Code } \\
2 \text { ) } \\
\end{array}$ & $\begin{array}{c}\text { Total } \\
\text { quantity (kg } \\
\text { or l) } \\
\end{array}$ & $\begin{array}{c}\text { Cost } \\
\text { (Shs/unit) }\end{array}$ & $\begin{array}{l}\text { Type } \\
\text { (Code } \\
3 \text { ) }\end{array}$ & & & & & \\
\hline $\begin{array}{l}\text { Coffee } \\
\text { (coffee } \\
\text { garden) }\end{array}$ & & & & & & & & & & & & & & & & \\
\hline & & & & & & & & & & & & & & & & \\
\hline & & & & & & & & & & & & & & & & \\
\hline & & & & & & & & & & & & & & & & \\
\hline & & & & & & & & & & & & & & & & \\
\hline & & & & & & & & & & & & & & & & \\
\hline & & & & & & & & & & & & & & & & \\
\hline
\end{tabular}

Code 2: $1=$ Cow dung $2=$ Cooking material refuse $3=$ Compost $4=0$ ther (specify)

Code 3: $1=$ =Round-up, $2=$ Gramaxon, $3=$ Dudu bitooke, $4=$ Weedmaster $5=$ Antkiller $6=0$ thers (specify)

${ }^{1}$ For Masaka district: Main season June-July and Fly season Dec-Jan; for Luwero district: Main season Dec -March and Fly in Aug-Oct

${ }^{2}$ If the farmer owns machinery ask for the cost of operation (e.g. fuel, hiring, maintenance). We are only interested in the variable costs.

Other additional costs incurred by the farmer (e.g. packaging). 
2.3 If yes, what was the total cost of constructing that drying place?

\section{SECTION 6: FARMING PRACTICES AND POST-HARVEST MANAGEMENT IN COFFEE}

\begin{tabular}{|c|l|}
\hline 1 & Do you intercrop or use crop cover on your coffee fields? \\
\hline 2 & Do you employ erosion measures (e.g. terraces) in your coffee gardens? \\
\hline 3 & On average, how many shade trees do you have in your gardens? (trees/acre) \\
\hline 4 & Did you cut any shade trees within the last 12 months? \\
\hline 5 & Do you use mulching/composting? \\
\hline 6 & Do you use organic pesticides? \\
\hline 7 & $\begin{array}{l}\text { Do you/ would you find it difficult to access chemical pesticides, herbizides, } \\
\text { fungicides? (e.g. because the products are too expensive, store too far away, etc.) }\end{array}$ \\
\hline 8 & Do you prune your coffee? \\
\hline 9 & Do you sometimes pick yellow or green cherries to reduce time spend on picking? \\
\hline 10 & Do you sometimes pick green cherries when you face urgent cash needs? \\
\hline 11 & How do you dry your coffee? [indicate most important one] \\
\hline 12 & How do you store your coffee after drying it? [indicate most important one] \\
\hline 13 & How do you ascertain the moisture content? [indicate most important one] \\
\hline 14 & What would you say is the factor constraining your coffee production most? \\
\hline 15 & Do you keep records for your coffee production activities? [if no, move to next section] \\
\hline $15 a$ & \\
\hline 15 & \\
\hline 16 & Who is usually responsible for record keeping? [indicate most important] \\
\hline 17 & Is someone helping that person in record keeping? [indicate most important] \\
\hline & Do you keep records on inputs? \\
\hline
\end{tabular}

\begin{tabular}{|c|c|}
\hline \multicolumn{2}{|l|}{\begin{tabular}{|c|}
$29 \_1$ \\
Currently
\end{tabular}} \\
\hline \begin{tabular}{|c|}
$29 \_1$ \\
Currently \\
\end{tabular} & \\
\hline & \\
\hline & \\
\hline & \\
\hline & \\
\hline & \\
\hline & \\
\hline & \\
\hline & \\
\hline & \\
\hline & \\
\hline & \\
\hline & \\
\hline & \\
\hline & \\
\hline & \\
\hline & \\
\hline & \\
\hline & \\
\hline & \\
\hline & \\
\hline & \\
\hline
\end{tabular}

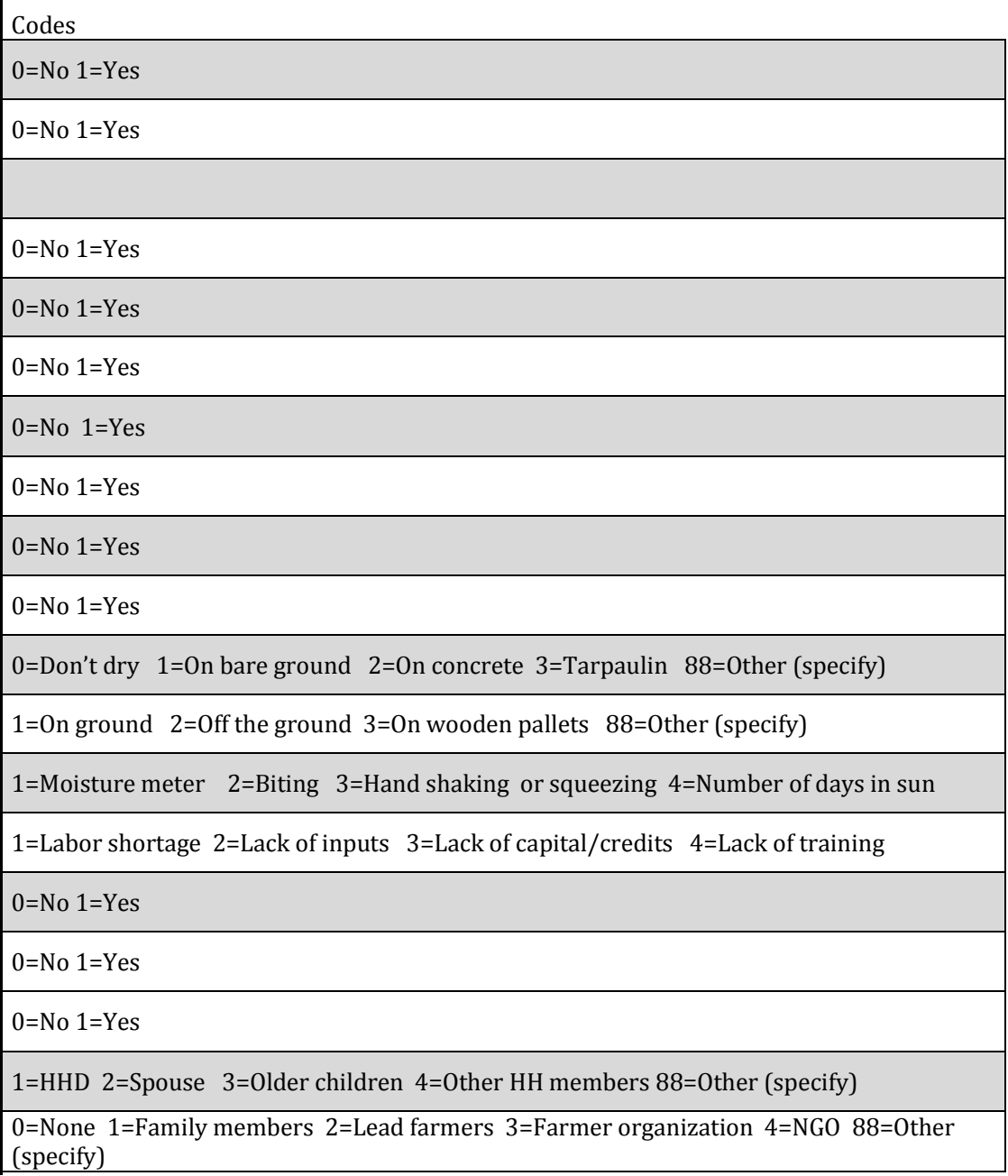


SECTION 7: LIVESTOCK PRODUCTION (C)

1.0 Please provide the following information on livestock owned by your $\mathrm{HH}$ in the past $\mathbf{1 2}$ months.

\begin{tabular}{|c|c|c|c|c|c|c|c|c|c|c|}
\hline \multirow{2}{*}{$\begin{array}{l}\text { Livestock and } \\
\text { livestock } \\
\text { products } \\
\text { (Code 1) } \\
\end{array}$} & \multirow{2}{*}{$\begin{array}{c}100 \\
\text { Number } \\
\text { currently } \\
\text { owned }\end{array}$} & \multirow{2}{*}{$\begin{array}{c}100 \_2 \\
\text { Who } \\
\text { owns it? } \\
\text { (Code 2) }\end{array}$} & \multirow{2}{*}{$\begin{array}{c}101 \\
\text { Estimated total current } \\
\text { value if all items sold } \\
\text { (Shs) }\end{array}$} & \multirow{2}{*}{$\begin{array}{c}103 \\
\text { Did you sell [item] in } \\
\text { the last } 12 \text { months } \\
(0=\text { No } 1=\text { Yes })\end{array}$} & \multirow{2}{*}{$\begin{array}{c}104 \\
\text { If yes, what was the } \\
\text { total value received }{ }^{2} \\
\text { (Shs) }\end{array}$} & 105 & $\begin{array}{c}106 \\
\text { tal Cost of } \mathrm{Pr}\end{array}$ & \begin{tabular}{|c|}
107 \\
\end{tabular} & 108 & \multirow{3}{*}{\begin{tabular}{|l} 
Code 1: \\
\\
30=Cow, bull, calve \\
31=Chicken \\
9=Rabbits \\
10=Goat
\end{tabular}} \\
\hline & & & & & & Fodder & Hired labor & Veterinary & Other costs & \\
\hline & & & Shs & & & & & & & \\
\hline & & & Shs & & & & & & & $\begin{array}{l}12=\text { Sheep } \\
13=\text { Donkeys }\end{array}$ \\
\hline & & & Shs & & & & & & & $16=$ Ducks \\
\hline & & & Shs & & & & & & & $88=0$ ther (specify) \\
\hline & & & Shs & & & & & & & $\begin{array}{l}\text { 18=Meat } \\
19=\text { Milk }\end{array}$ \\
\hline & & & Shs & & & & & & & $\begin{array}{l}20=\text { Eggs } \\
21=\text { Honey }\end{array}$ \\
\hline & & & Shs & & & & & & & $88=0$ ther (specify) \\
\hline
\end{tabular}

\section{SECTION 8: ASSET OWNERSHIP (D)}

1.0 Please provide the following information on assets owned by this HHD in the past 12 months?

\begin{tabular}{|c|c|c|c|c|c|c|c|c|c|}
\hline \multirow{2}{*}{\multicolumn{2}{|c|}{ Item }} & \multirow{3}{*}{$\begin{array}{c}109 \\
\begin{array}{c}\text { Units currently } \\
\text { owned }\end{array} \\
\end{array}$} & \multirow{3}{*}{$\begin{array}{c}110 \\
\begin{array}{c}\text { Estimated total current value } \\
\text { (Shs) all items }\end{array}\end{array}$} & \multirow{3}{*}{$\begin{array}{c}\text { 111_1 } \\
\text { Who }{ }^{1} \text { owns it? } \\
\text { (Code } 1 \text { ) }\end{array}$} & \multirow{2}{*}{\multicolumn{2}{|c|}{ Item }} & \multirow{3}{*}{$\begin{array}{c}109 \\
\begin{array}{c}\text { Units currently } \\
\text { owned }\end{array} \\
\end{array}$} & \multirow{3}{*}{$\begin{array}{c}110 \\
\begin{array}{l}\text { Estimated total current value (Shs) } \\
\text { all items }\end{array} \\
\end{array}$} & \multirow{3}{*}{$\frac{111 \_1}{\text { Who }^{1} \text { owns it? (Code 1) }}$} \\
\hline & & & & & & & & & \\
\hline 4 & Wheel barrow & & & & 14 & Generator & & & \\
\hline 5 & $\begin{array}{l}\text { Knapsack } \\
\text { sprayer }\end{array}$ & & & & 15 & $\begin{array}{l}\text { Furniture (chairs, } \\
\text { tables) }\end{array}$ & & & \\
\hline 7 & Motor vehicle & & & & 16 & Television & & & \\
\hline 8 & Motor cycle & & & & 17 & Radio & & & \\
\hline 9 & Bicycle & & & & 18 & Mobile Phones & & & \\
\hline 30 & Pangas, hoes & & & & 19 & Sleeping beds & & & \\
\hline 11 & Pruning saw & & & & 20 & Water pump: & & & \\
\hline 13 & Water tanks & & & & 21 & Jewelry & & & \\
\hline 88 & Other (specify) & & & & 88 & Other (specify) & & & \\
\hline
\end{tabular}

Code 1: 1=HHD 2=Spouse 3=Jointly 4=N.A. 88=0ther 
2.0 Provide the following information on HH assets currently owned.

\begin{tabular}{|c|c|c|c|}
\hline & \multirow{2}{*}{ Current ownership } & \\
\hline 1 & Type of dwelling & & $\begin{array}{l}\begin{array}{l}1=\text { Mud hut with grass thatching } 2=\text { Mud hut with asbestos/iron roof } 3=\text { Brick house with grass thatching } 4=\text { Brick house with } \\
\text { asbestos/iron roof }\end{array} \\
\end{array}$ \\
\hline 2 & Tenure status of dwelling & & $1=0$ wn with title deeds $2=0$ wn without title deeds $3=$ Rented $\quad 4=$ Borrowed without pay $88=0$ ther (specify) \\
\hline 3 & Total number of rooms owned & & \\
\hline 4 & Type of toilet & & 1=Bush $2=$ Flush $3=$ =Ventilated latrine $\quad 4=$ Pit latrine $\quad 88=0$ ther (specify) \\
\hline 5 & Main source of drinking water & & $1=$ Private tap $2=$ Public tap/borehole $4=$ River, stream, lake, pond, well, springs $\quad 5=$ Rain water $\quad 88=0$ ther (specify) \\
\hline 6 & Do you usually treat your drinking water? & & $\begin{array}{l}=\text { No } \quad 1=\text { Boil } \quad 2=\text { =Chlorine/bleach } 3=\text { Use traditional herbs } \quad 4=\text { Use chemicals (water guard, liquid) } \quad 5=\text { =Filter/sieve } \quad 6=\text { Decant } \\
7=\text { Other (specify) }\end{array}$ \\
\hline 7 & Main source of lighting & & $2=$ Paraffin lantern $3=$ =Candles $\quad 4=$ Wick Lamp $88=$ =ther (specify) \\
\hline 8 & Main type of cooking fuel & & $5=$ =Paraffin/Kerosene $\quad 6=$ Solar $\quad 7=$ Biogas $\quad 88=0$ ther (specify) \\
\hline
\end{tabular}

2.9 Does this HH have any close wealthy ancestors (parents, uncles, aunties, nieces, in-laws or nephews)? 1=Yes, $0=$ No

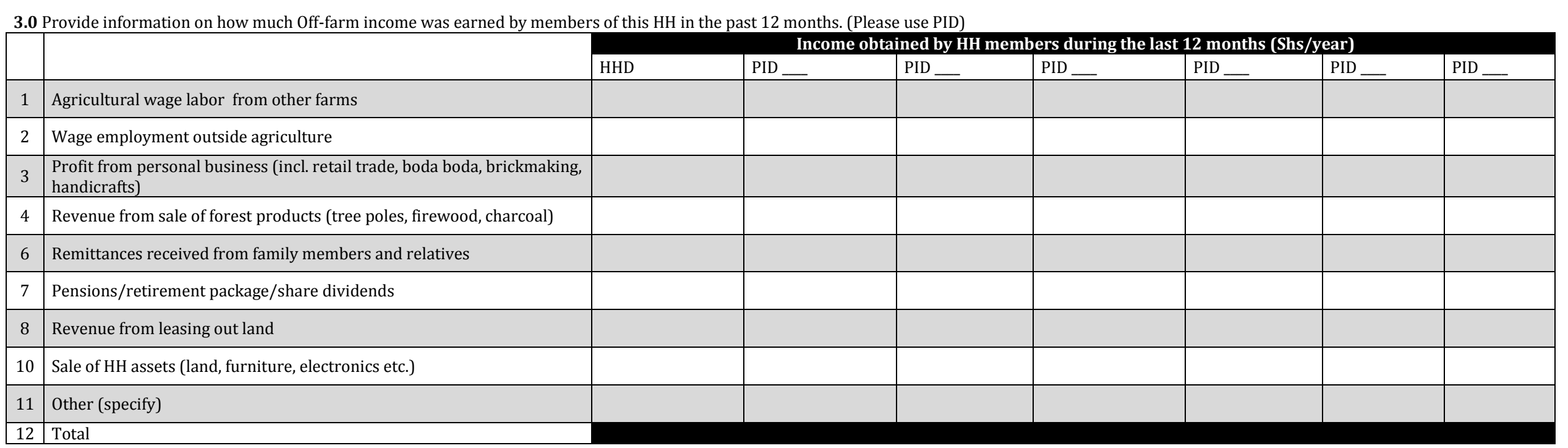


SECTION 9: NON-FOOD EXPENDITURE (H)

1.0 Consider the last 12 months, generally how much has your HH spent on the items listed in a typical month, term, or year (see specification indicated for each item)? (Enter zero if nothing is consumed. Enter 99 if they don't know)

\begin{tabular}{|c|c|c|c|c|c|}
\hline P & Item & $\begin{array}{c}162 \\
\text { Fxnenditure }(\text { total value in }\end{array}$ & \multicolumn{2}{|r|}{ Item } & 163 \\
\hline 1 & Rentals (house) per month & & 8 & Telephone bills (including mobile) per month & \\
\hline $1 \mathrm{~b}$ & Rentals land per year & & 9 & Ceremonies (church, weddings, festivals, burials) per year & \\
\hline 2 & Kitchen utensils (pots, pans, plates, cutlery) per year & & 10 & Firewood/Charcoal per month & \\
\hline 3 & HH furniture (beds, tables, chairs) per year & & 11 & Remittances or transfers to other individuals per year & \\
\hline 4 & Clothing and footwear (fabric, clothes, towels, shoes) per year & & 12 & Repairs of machinery, equipment, housing per year & \\
\hline 5 & Electricity, Gas, Paraffin, Biogas (for cooking and light) per month & & 13 & Amount paid as interest, other fees, amagoba per month & \\
\hline 6 & Education, books, school fees, uniforms per term & & 14 & Public transport (boda-boda, taxi, bus) per month & \\
\hline 7 & Health care (consultation fees, medicines, spectacles) per year & & 30 & Hygiene and beauty products, cosmetics per year & \\
\hline 15 & Membership fees (COOP, Fmr groups, Burial societies per year & & 88 & Other major non-food items (specify) per month & \\
\hline
\end{tabular}

2.0 In the past 12 months, how much did your HH spend on the items for individual HH members under 25? (Use PID; write down "0" if the item/service is provided for free and " 88 " if not applicable)

\begin{tabular}{|c|c|c|c|c|c|}
\hline $\begin{array}{l}\text { HH member } \\
\text { PID }\end{array}$ & $\begin{array}{l}\text { How much do you pay for school } \\
\text { fees and uniforms for [NAME]? }\end{array}$ & $\begin{array}{l}\text { For extra classes, books, and stationary } \\
\text { (exercise books, pens, etc.)?? }\end{array}$ & $\begin{array}{l}\text { For transport to } \\
\text { school? }\end{array}$ & $\begin{array}{l}\text { For health related costs (vaccinations, medical } \\
\text { consultation, medicine, other health measures) }\end{array}$ & For clothing and footwear? \\
\hline & & & & & \\
\hline & & & & & \\
\hline & & & & & \\
\hline & & & & & \\
\hline & & & & & \\
\hline & & & & & \\
\hline & & & & & \\
\hline & & & & & \\
\hline
\end{tabular}

\section{SECTION 10: FOOD EXPENDITURE (GI)}

1 On average, how many people were present in the last 7 days? In this section children are defined as persons under 18 years.
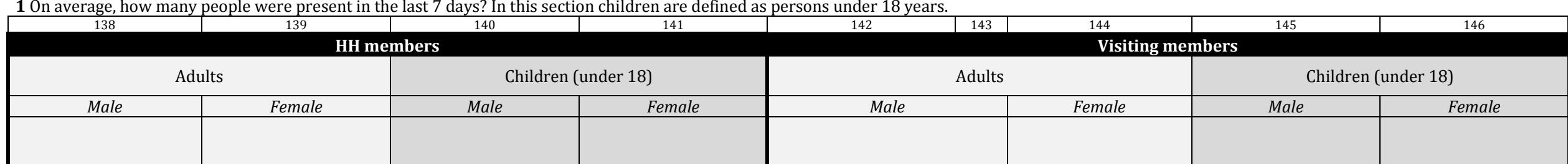

2.0 In the past 7 days indicate how much of the following food items your HH consumed and the value in Shs. (For all food consumed, including own-produced, bought, gifts and from food aid program, by all people living in $\mathrm{HH}$ ) 


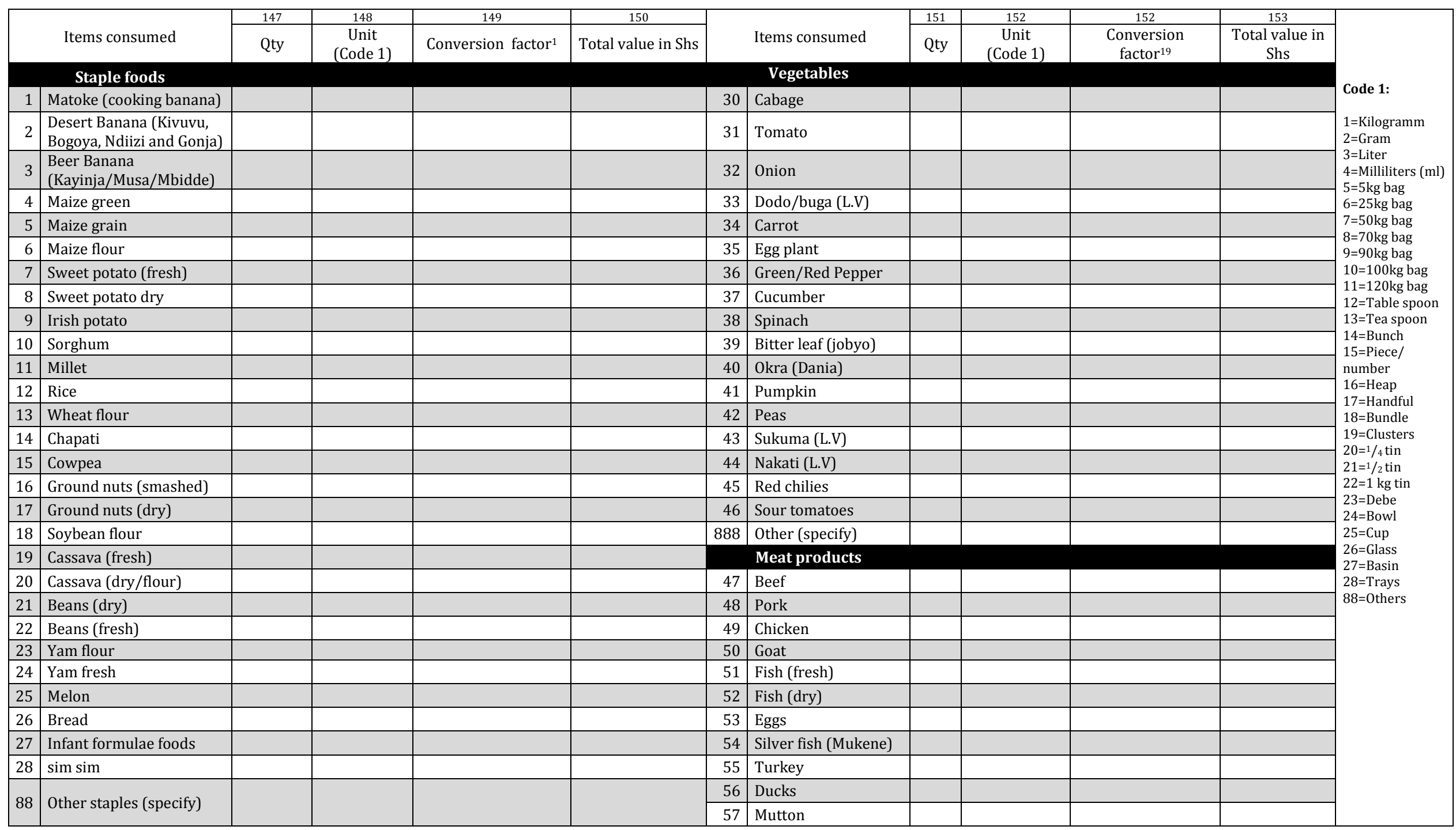

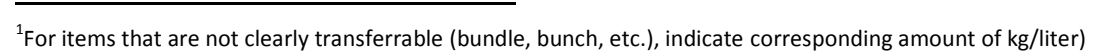


2.0 Continued (In the past 7 days indicate how much of the following food items your HH consumed and the value in Shs. (For all food consumed, including own-produced, bought, gifts and from food aid program, by all people living in $\mathrm{HH}$ )

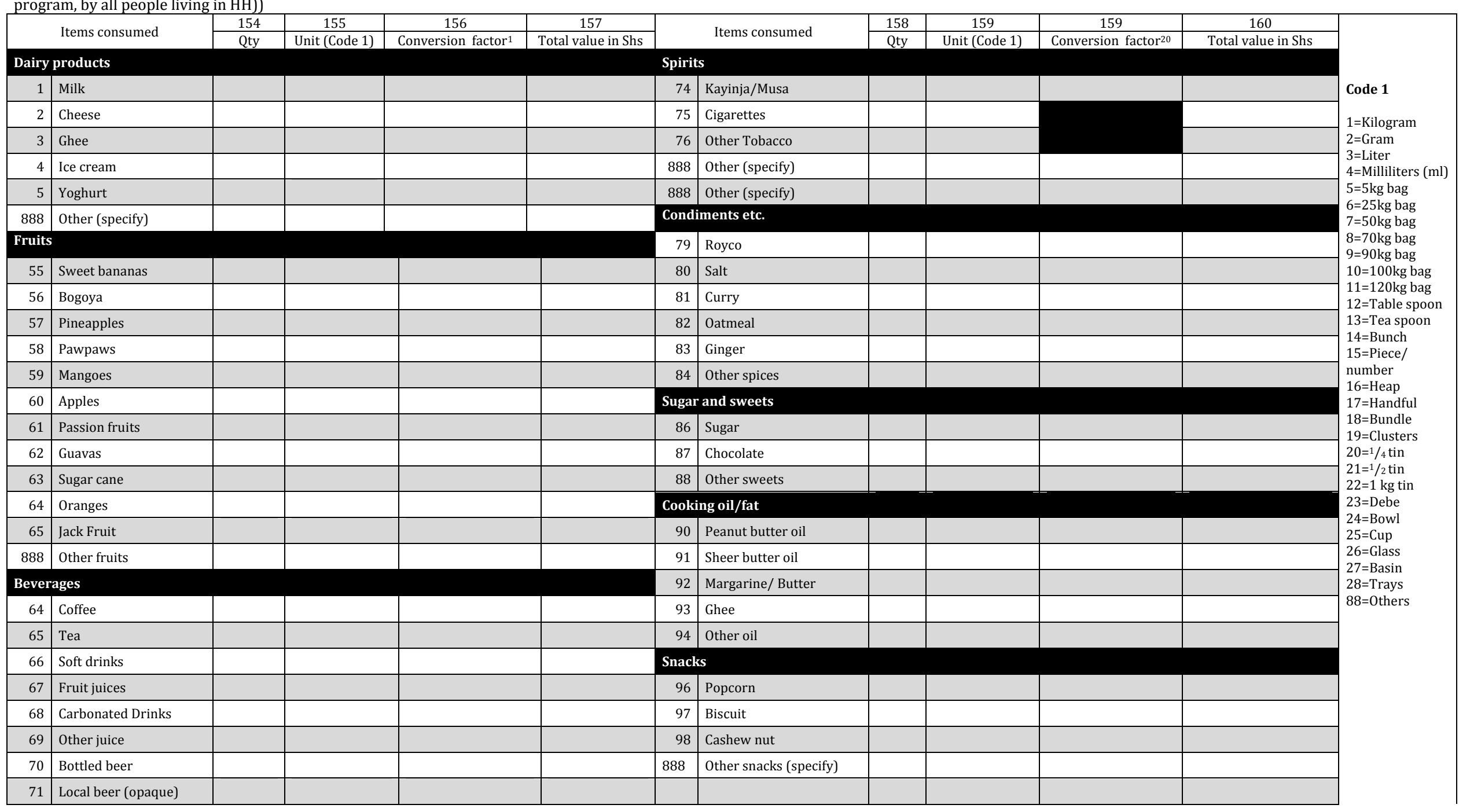

$\overline{{ }^{1} \text { For items that are not clearly transferrable (bundle, bunch, etc.), indicate corresponding amount of } \mathrm{kg} / \text { liter }}$ 
$\mid$

3.1 During the past 7 days, did any of the $\mathrm{HH}$ members eat food away from home (e.g. in schools, restaurants, during ceremonies)?

3.2 If yes, indicate the number of times they ate food away and the value of meals in the past 7 days.

\begin{tabular}{|c|c|c|c|c|c|c|c|c|c|c|c|}
\hline & & HHD & PID:_____ & PID:___ & PID:____ & PID:_____ & PID:____ & PID: & PID:___ & PID:___ & PID:____ \\
\hline 1 & Number of times & & & & & & & & & & \\
\hline 2 & $\begin{array}{l}\text { Value of meals (Shs) (total for all } 7 \\
\text { days) }\end{array}$ & & & & & & & & & & \\
\hline 3 & $\begin{array}{l}\text { Which foods does member normally } \\
\text { eat (Code } 1 \text { ) }\end{array}$ & & & & & & & & & & \\
\hline
\end{tabular}

Code 1: 1=Beans 2=Posho 3=Maize porridge 4=Meat 5=Fish 6=Rice 88=0ther (specify)

\section{SECTION 11: SERVICES (I)}

1.0 How far in kilometers would you have to travel to access the following services? (Indicate distance to nearest source in $\mathrm{km}$ )

\begin{tabular}{|c|c|c|c|c|c|}
\hline & & Distance to nearest source $(\mathrm{km})$ & & & Distance to nearest source (km) \\
\hline 1 & Financial credit & & 12 & Tarmac road (and bus connection to Kampala) & \\
\hline 22 & Micro-credit and saving facility & & 15 & Electricity & \\
\hline 10 & Commercial bank & & 16 & Water source for $\mathrm{HH}$ use & \\
\hline 3 & Input market for coffee & & 14 & Watering source (livestock) & \\
\hline 4 & Output market for coffee (different from farm-gate) & & 18 & Internet & \\
\hline 5 & Coffee collection center & & 20 & Religious facilities & \\
\hline 6 & Extension office/r & & 22 & Hospital, clinic or doctor & \\
\hline 21 & Coffe nurseries run by fellow farmers & & 7 & Primary school & \\
\hline 9 & Source of coffee seedlings & & 8 & Secondary school & \\
\hline 11 & Promoter lead farmer for coffee & & 19 & University & \\
\hline
\end{tabular}


1.1 Did anyone in this HHD own a mobile phone handset?

$(0=$ No $1=$ Yes $) \rightarrow$ If NO, move to the next section!

1.2 Which year did the earliest user of a mobile phone in this HH start using a mobile phone? [indicate year]

\begin{tabular}{|c|c|c|c|c|c|c|c|}
\hline & & Currently (2015) & in 2012 & & & Currently & in 2012 \\
\hline 1 & $\begin{array}{l}\text { Is/Was your HH's area covered with mobile phone network? } \\
(\mathrm{No}=0 \text { Yes=1) }\end{array}$ & & & 5 & $\begin{array}{l}\text { How far was the (nearest) shop/place where members of this HH could } \\
\text { buy/bought a mobile phone handset? }\end{array}$ & $\mathrm{km}$ & $\mathrm{km}$ \\
\hline 2 & $\begin{array}{l}\text { Is/Did this HH's area have mobile phone telecom service center } \\
\text { in nearest town? (No=0 Yes=1) }\end{array}$ & & & 6 & $\begin{array}{l}\text { How far was the (nearest) shop/place where members of this HH } \\
\text { could/bought airtime? }\end{array}$ & $\mathrm{km}$ & $\mathrm{km}$ \\
\hline 3 & $\begin{array}{l}\text { Did sometimes members of this HH use neighbors' or friends' } \\
\text { phones for calls or messages? [ALL] (No=0 Yes }=1 \text { ) }\end{array}$ & & & 7 & $\begin{array}{l}\text { How far was the (nearest) shop/place where members of this HH } \\
\text { could/recharged the phone battery? }\end{array}$ & $\mathrm{km}$ & $\mathrm{km}$ \\
\hline 4 & $\begin{array}{l}\text { Of the TEN neighbors to this HH, how many of them have } \\
\text { mobile phones? (indicate number) }\end{array}$ & & & 8 & $\begin{array}{l}\text { Are/were there mobile phone or computer use training centers in your } \\
\text { HH's vicinity? }\end{array}$ & & \\
\hline
\end{tabular}

3.0

\begin{tabular}{|c|c|c|c|}
\hline & & Currently & in 2012 \\
\hline 1 & $\begin{array}{l}\text { Who in this HH owned a mobile phone? (For only those who had, record their PIDs under respective } \\
\text { years in second and third column on the right). }\end{array}$ & $\begin{array}{ll}\text { 1. } & - \\
\text { 2. } & \\
\text { 3. } \\
\text { 4. } & - \\
\end{array}$ & $\begin{array}{ll}\text { 1. } & - \\
\text { 2. } & - \\
\text { 3. } & \\
\text { 4. } & - \\
\end{array}$ \\
\hline 2 & $\begin{array}{l}\text { Generally, how many times did (PERSON (record PID in second and third column under respective } \\
\text { year)) use mobile phone per day? (Making and receiving calls)? }\end{array}$ & $\begin{array}{ll}\text { 1. } & - \\
2 . & - \\
\text { 3. } & - \\
\text { 4. } & - \\
\end{array}$ & $\begin{array}{ll}\text { 1. } & - \\
\text { 2. } & - \\
\text { 3. } & - \\
\text { 4. } & - \\
\end{array}$ \\
\hline 3 & How many network bars (reflecting network strength) is/would your mobile phone show? & & \\
\hline 4 & $\begin{array}{l}\text { Which Mobile Phone network provider is } \backslash \text { was the HHD subscribed to? } \\
\text { (1=MTN } 2=\text { Airtel } 3=\text { UTL } 4=\text { KT } 5=\text { =range } 6=\text { Smile } 7=\text { Warid } \quad 8=\text { Vodafone } 9=0 \text { thers (specify)) }\end{array}$ & & \\
\hline 5 & $\begin{array}{l}\text { How does } \backslash \text { did the HHD rate the quality of telephone network coverage here? } \\
(1=\text { very good } 2=\text { Good } 3=\text { Fair } 4=\text { Poor } 5=\text { Very poor })\end{array}$ & & \\
\hline 6 & On average, how much did the HHD spend in total on airtime per month? & Shs & Shs \\
\hline
\end{tabular}


1 Does any member in this HH use mobile money services?

$(0=\mathrm{No} \quad 1=\mathrm{Yes}) \rightarrow$ If $\mathrm{NO}$, move to the next section!

2 Which year did the earliest user of MM in this HH start using mobile money services? Year

3 Is/Was your HH's area covered with MM service network?

4 Of TEN of this HH's nearest neighbors, how many in this village use MM?

5 Did this HH's area have a MM service center in the nearest town? (1=Yes, $0=$ No)

6 How far was the nearest MM service center where members of this HH could withdraw or deposit money into M-account?

7 How much would it cost (one way trip) for a member from this HH to reach that MM center?

8 Did any member of this HH, ever used friends' or neighbors MM services? (No=0 Yes=1)

$8 \mathrm{~b}$ If you received a text message could you open and read it?

9 Who in this HH used Mobile Money? [Record only PIDs for those who used MM in the second and third columns on the righ].

10 On average, how much money did/would the HHD receive per year?

11 On average, how much money did/would the HHD send per year?

12 Which mobile money operator is the HHD subscribed to?

12 1=MTN 2=Airtel 3=UTL 4=Smile 5=Orange 6=Easy Money 7=Vodafone 8=K2 9=Warid 10=Others (specify)

13 Does/did the HHD know how much he/she is/was charged to send 10,000 UGX via MM services? (No=0 Yes=1)

\begin{tabular}{|c|c|c|c|}
\hline & & Currently & in 2012 \\
\hline 14 & $\begin{array}{l}\text { What activity did the HHD mostly do via MM services? (tick ONE) (1=Withdrawing money } 2=\text { Sending Money } 3=\text { Paying bills } 4=\text { Paying school fees } 5=\text { Saving money } \\
6=\text { Buying airtime } 7=\text { Buying inputs } 8=\text { =Paying laborers } 9=\text { Transfer money } \quad 10=0 \text { thers (specify)) }\end{array}$ & & \\
\hline 15 & $\begin{array}{l}\text { Generally, for WHAT purpose did the HHD receive most remittances via MM services? (tick ONE) } \\
\begin{array}{ll}(1=\text { Paying school fees } \quad 2=\text { Given birth } \quad 3=\text { Had food shortage } \quad 4=\text { Lost (dead) relative } 5=\text { =Social group function } \quad 6=\text { General HH financial aid } \quad 7=0 \text { thers (specify)) }\end{array}\end{array}$ & & \\
\hline 16 & To whom did the HHD mostly send money/remittances via MM services? Tick ONE (Code 6) & & \\
\hline 17 & From whom did the HHD mostly receive money/remittances via MM services? Tick ONE (Code 6) & & \\
\hline 18 & Averagely, how distant (in Km) was the person the HHD mostly sent/received money? & & \\
\hline 19 & Did the HHD ever receive/pay back any money from the person he/she mostly sent/received money via MM services? & & \\
\hline 20 & Did the HHD sometimes channel MM remittances sent/planned/aimed for a particular purpose to other abruptly urgent HH needs? $(\mathrm{No}=0$, Yes=1) & & \\
\hline
\end{tabular}

Code 6: 1=Spouse 2=Son(s) 3=Daughter(s) 4=Friend(s) 5=Farmer group members 6=Father 7=Mother 8=Politician 9=Farm laborers 10=Business partners 11=0thers (specify) 
22 Do you sometimes have severe $\mathrm{HH}$ misunderstandings? 1=Yes, $0=\mathrm{No}$

\section{SECTION 14: ANTHROPOMETRY}

Explain selection and measurement procedure to respondents. Select one male and one female infant (2-5 years) for the anthropometric measurement. Use random selection procedure if there are more than two potential infants. Also select the parents of the selected infants. If one or all of them are not present -or if the mother of the selected children is pregnant - use random selection procedure to select another male and/or female HH member.

\begin{tabular}{|c|c|c|c|c|c|}
\hline & & Female infant & Male infant & Female adult & Male adult \\
\hline 1 & Name & & & & \\
\hline 2 & Person ID & & & & \\
\hline 3 & Age & & & & \\
\hline 4 & Name of the mother & & & & \\
\hline 5 & Name of the father & & & & \\
\hline 6 & $\begin{array}{l}\text { Is the selected female adult } \\
\text { pregnant? (If yes, do not } \\
\text { continue. Select another } \\
\text { female HH member!) }\end{array}$ & & & $-(0=$ No $1=$ Yes $)$ & \\
\hline
\end{tabular}

\begin{tabular}{|c|c|c|c|c|c|c|c|c|c|}
\hline & $x^{2}$ & Female infant & Male infant & Female adult & Male adult & \multirow{7}{*}{$\begin{array}{l}\text { Code 1: } \\
1=\text { Malaria } \\
2=\text { Diarrhea } \\
\text { 3=Fever } \\
4=\text { Stomach ache } \\
5=\text { =omiting } \\
6=\text { Flue/cold } \\
7=\text { =eadache } \\
8=\text { Skin problems } \\
9=\text { Eye problem } \\
\text { 10=Ear/nose/ throat } \\
\text { 11=Pain when } \\
\text { passing urine } \\
12=\text { Typhoid } \\
13=\text { Pneumonia } \\
14=\text { Fainting } \\
15=\text { Intestinal worms } \\
88=\text { =ther (specify) }\end{array}$} & \multirow{7}{*}{$\begin{array}{l}\text { Code 2: } \\
\text { (If more than one, } \\
\text { record the two most } \\
\text { severe) } \\
\\
\text { 1=Diabetes } \\
2=\text { Hypertension } \\
3=\text { Cardiovascular/ heart } \\
\text { disease } \\
4=\text { Kwashiorkor } \\
5=\text { Cancer } \\
\text { 6=Rickets } \\
7=\text { If not at birth } \\
\text { (blindness) } \\
8=\text { Goiter } \\
9=\text { Gout } \\
10=\text { Bad teeth } \\
11=\text { HIV/Aids } \\
12=\text { Tuberculosis }\end{array}$} & \multirow{7}{*}{$\begin{array}{l}\text { Code } 3: \\
1=\text { All of the } \\
\text { time } \\
2=\text { Most of the } \\
\text { time } \\
3=\text { =Some of the } \\
\text { time } \\
4=\text { None/almos } \\
\text { t none of the } \\
\text { time }\end{array}$} & \multirow{7}{*}{$\begin{array}{l}\text { Code 4: } \\
0=\text { Never } \\
\text { 1=Sometimes } \\
\text { 2=Frequently } \\
88=\text { Not asked } \\
\text { because } \\
\text { respondent } \\
\text { may be } \\
\text { offended }\end{array}$} \\
\hline 1 & $\begin{array}{l}\text { During the last } 2 \text { weeks, did any selected } \mathrm{HH} \\
\text { member suffer from an acute illness/condition that } \\
\text { resulted in weight loss? (Code } 1 \text { ) }\end{array}$ & & & & & & & & \\
\hline 2 & $\begin{array}{l}\text { Does this member suffer from any chronic diseases? } \\
\text { (Code 2) }\end{array}$ & & & & & & & & \\
\hline 3 & $\begin{array}{l}\text { Does this infant have a pot belly or skin/hair } \\
\text { changes? }{ }^{1}\end{array}$ & & & & & & & & \\
\hline 4 & $\begin{array}{l}\text { How often does your occupation/work activity } \\
\text { require lots of physical effort in a typical week? } \\
\text { (Code 3) }\end{array}$ & & & & & & & & \\
\hline 5 & Do you drink alcohol? (Code 4) & & & & & & & & \\
\hline 6 & $\begin{array}{l}\text { Did you ever regularly smoke cigarettes? }(0=\text { No } \\
1=\text { Yes) }\end{array}$ & & & & & & & & \\
\hline
\end{tabular}

${ }^{1}$ May be indicating Kwashiorkor (protein energy malnutrition with the following symptoms: edema, large belly that sticks out, skin and hair changes). 


\begin{tabular}{|c|c|c|c|c|c|}
\hline & & & & & \multirow{3}{*}{ Male adult } \\
\hline \multicolumn{2}{|c|}{ 3.0 Report result of measurements } & Female infant & Male infant & Female adult & \\
\hline 1 & Calibration weight & \multicolumn{3}{|c|}{ ok } & \\
\hline 2 & Weight in kg & $\mathrm{kg}$ & $\mathrm{kg}$ & $\mathrm{kg}$ & $\mathrm{kg}$ \\
\hline 3 & Type of cloth ( $0=$ =Light clothing $1=$ Heavy clothing) & & & & \\
\hline 4 & Height in $\mathrm{cm}$ & $\mathrm{cm}$ & $\mathrm{cm}$ & $\mathrm{cm}$ & $\mathrm{cm}$ \\
\hline 5 & Hip circumference in cm & & & $\mathrm{cm}$ & cm \\
\hline 6 & Waist circumference in $\mathrm{cm}$ & & & $\mathrm{cm}$ & $\mathrm{cm}$ \\
\hline
\end{tabular}

4.1 Is any $\mathrm{HH}$ member currently pregnant?

4.2 PID: months:

( $0=$ No $1=$ Yes) (If yes, indicate PIDs and moths of pregnancy)

PID: months:

PID:

$(0=$ No $1=Y e s)$

5.1 Is any member within the $\mathrm{HH}$ currently breastfeeding? _[number of breastfeeding women]

6.1 In 2012 (May to August), was any HH member pregnant? $(0=$ No $1=$ Yes $)$

6.2 If yes, how many HH members? [number of pregnant women]

7.1 In 2012 (May to August), was any HH member breastfeeding? $(0=$ No $1=$ Yes $)$

7.2 If yes, how many $\mathrm{HH}$ members? [number of breastfeeding women] 


\section{Part 2: Gender disaggregated part (Make sure to interview the male and female respondent separately!)}

Name of the respondent:

\section{SECTION 15: CHOICE EXPERIMENT}

1.1 Carefully explain the procedure using choice card No. 0 and translate the description of the pictures, record choices made by the respondent.

\begin{tabular}{|l|l|c|c|c|}
\hline & & \multicolumn{2}{|c|}{ Indicate respondent's choice here with a 1 (put 0 for the options not chosen) } \\
\hline & Choice set number & Option 1 & Option 2 & Option 3 \\
\hline Choice set & & & & \\
\hline Choice set & & & & \\
\hline Choice set & & & & \\
\hline Choice set & & & & \\
\hline Choice set & & & & \\
\hline Choice set & & & & \\
\hline
\end{tabular}

\section{SECTION 16: ATTITUDES TOWARDS CERTIFICATION}

2.0 Is or was this $\mathrm{HH}$ ever certified?

( $(0=$ No $1=$ Yes $) \rightarrow$ If no, move to question 3 .

2.1 Do you think your $\mathrm{HH}$ benefits from being certified?

2.2 Do you feel you personally benefit from certification?

$(0=$ No $1=$ Yes $)$

2.3 If yes, what is the GREATEST benefit for YOU ? (Code 1)

$(0=$ No $1=$ Yes $)$ ode 1: $1=$ =Economic benefits (higher price, more income, better market access) $2=$ Training opportunities $3=$ Social benefits (bargaining power, trust among members, collective activities, development projects, being famous) $4=0$ ther (specify)

2.4 Would you say that certification is costly for your HH (e.g. because of required investments)? $(0=$ No $1=$ Yes $)$

2.5 Do you find it difficult to meet certification requirements such as rules regarding the use of pesticides, prohibition of child and prisoner labor, or coffee quality requirements?

3.0 Only ask NON certified respondents that were NEVER certified. $\rightarrow$ If the $\mathrm{HH}$ is certified, move to next section.

3.1 Did you ever consider participating in a certification scheme such as UTZ, Fairtrade, Organic?

3.2 Did anyone ever offer you support to become certified (e.g. farmer organization or NGO) $(0=$ No $1=$ Yes $)$

3.3 Do you think your HH could benefit from certification? $(0=$ No $1=$ Yes $)$

3.4 Do you think you could personally benefit from certification? $(0=$ No $1=$ Yes $)$ Code 1: 1=Economic benefits (higher price, more income, better market access) $2=$ Training opportunities $3=$ =Social benefits (bargaining power, trust among members, collective activities, development projects) 4=0ther (specify)

3.6 Do you feel certification would be too expensive for your HH (because it requires certain investments)?
3.7 Would you find it difficult to meet certification requirements such as rules regarding the use of pesticides, prohibition of child and prisoner labor, or coffee quality requirements? $(0=$ No $1=$ Yes $)$ 


\section{SECTION 17: SOCIAL NETWORKS, ACCESS TO SERVICES (S.I \&S.L)}

\begin{tabular}{|c|c|}
\hline 1 & Are you a (registered) member of any group? \\
\hline 2 & Is any other $\mathrm{HH}$ member a member of any group? \\
\hline 3 & $\begin{array}{l}\text { Did you hold any leadership position/role in the community within } \\
\text { the last } 3 \text { years? }\end{array}$ \\
\hline 4 & $\begin{array}{l}\text { Did any other member of this } \mathrm{HH} \text { hold any leadership position/role } \\
\text { in the community within the last } 3 \text { years? }\end{array}$ \\
\hline 5 & $\begin{array}{l}\text { Are you able to receive credit for agricultural production when you } \\
\text { need such credit? }\end{array}$ \\
\hline 6 & $\begin{array}{l}\text { Has anyone in your } \mathrm{HH} \text { in fact taken any loan or borrowed cash/in- } \\
\text { kind in the past } 12 \text { months? }\end{array}$ \\
\hline 7 & $\begin{array}{l}\text { Who makes the decision about what to do with the borrowed } \\
\text { money/item? (Code 4) }\end{array}$ \\
\hline 8 & Do you, personally, (not your $\mathrm{HH}$ ) have a savings account? \\
\hline 9 & Did you have a savings account in $2012 ?$ \\
\hline 10 & Does any other HH member have a savings account? \\
\hline
\end{tabular}

0=No 1=Yes

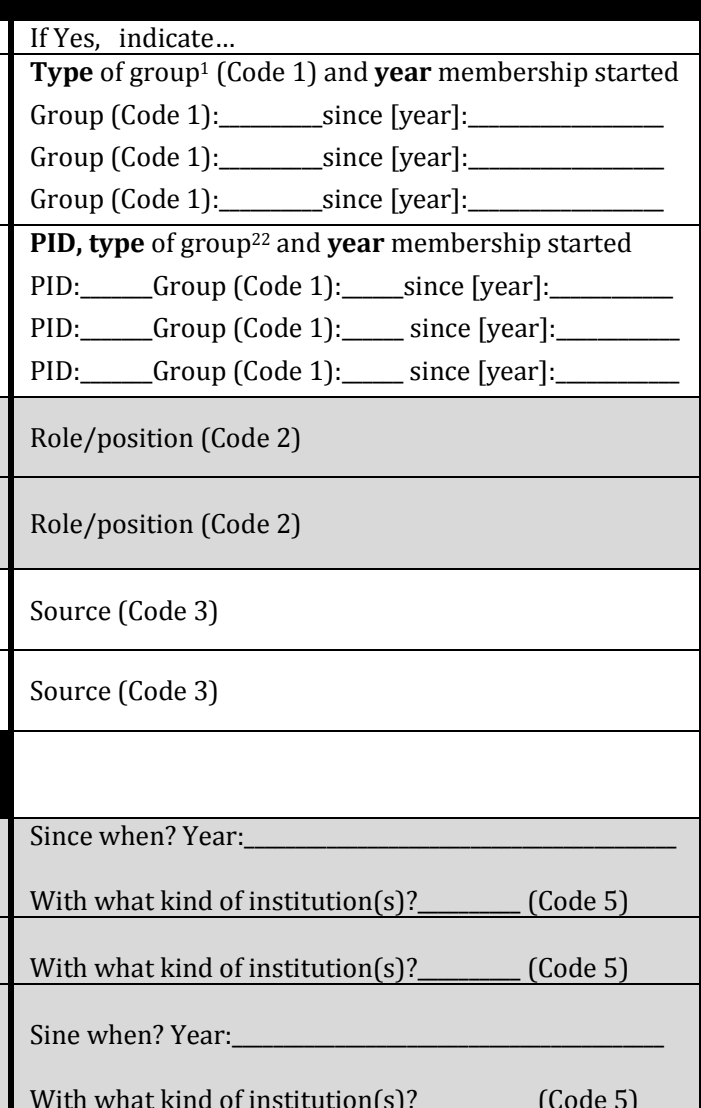

Codes

Code 1

$1=$ Farmer organization

$2=$ Women's group

$3=$ Credit and savings group

4=Religious groups

ce groups (e.g. burial societies)

7 Thade and business association

$$
\text { Code 2 }
$$

4=Religious organization

5=Farmer organization/group

6=Extension officer

$\begin{array}{ll}\text { Committee } & 7=\text { =Cultural-spirit } \\ & 8=\text { Lead farmer }\end{array}$

Code 3:

$1=$ Farmer group

$2=$ Cooperative

$88=0$ thers (specify)

6=Local agro dealers
$7=$ Farmers

$4=$ Local bank

Code 4: $\quad 3=$ Jointly

$1=\mathrm{HHD}$

$2=$ Spouse

(e. Centenary)

$2=$ Semi-formal financial institution (e.g. MFI, SACCO)

$3=$ Mobile Telephone Operator

$4=0$ ther (specify)

5.0 Agricultural training and information

5.1 Are you able to receive agricultural information when you need such information? $(0=$ No $1=$ Yes $)$

5.2 If yes, what is the most important source (Code 5) (allow multiple answers, but START with the most important) Code 5: 1=Extension staff 2 =Extension bulletins $4=0$ ther farmers $5=$ Radio $6=$ TV $\quad 7=$ Newspaper $8=$ Mobile phone alerts $88=0$ ther (specify)

${ }^{1}$ If multiple, indicate 3 most important ones. 
5.3 Did you interact with an extension officer on agricultural related issues in the last 12 months?

5.4 Did you attend any field days or demonstrations on coffee farming in the last 12 months?

5.5 Within the last 12 months, did you participate in any training on the following issues:

1. Pest regulation/use of (organic) pesticides

2. Safe handling and storage of pesticides, usage of protective clothing 3. Measures to improve soil fertility, measures to avoid soil erosion (e.g. fertilizers, compost, terraces)

4. Measures to improve coffee quality: coffee harvesting, drying, storage 6. Gender equality 7. Health, nutrition 8. [ONLY ask CERTIFIED farmers ]General certification requirements (regulations of Fairtrade/Organic/UTZ) $6 \quad$ Within the last 12 months, did you participate in any community meeting or farmer group meeting? 9. Any other training? (Specify) Code 6: $1=$ Farmer organization $2=\mathrm{HRNS}$ (only Luwero) $3=0$ ther actors (incl. government or NGOs)

7.0 Speaking in the public

1 Do you feel comfortable speaking up during community or farmer group/organization meetings to share your point of view?

2 Do you feel comfortable speaking up in public to protest the misbehavior of authorities or elected officials?

\begin{tabular}{l|l|l|l}
$0=$ No $1=$ Yes & No. of times & Provider (Code 6) \\
\hline & &
\end{tabular}

8.0 How are travel arrangements in your HH? Do you travel?

1 Do you travel to markets?

2 Do you travel to relatives?

3 Do you travel to health centers/doctor?

4 Do you travel to Masaka/Luwero town?

5 Do you travel to Kampala?

Code 8: 1=By HHD 2=By male HH member 3=By female HH member 88=0ther (specify)

\section{SECTION 18: INTRA HOUSEHOLD DECISION MAKING}

\section{Do you agree with the following statements?}

\begin{tabular}{|l|l|l|r|}
\hline Yes, alone & Yes, accompanied by...(Code 8) & No/never & Distance (min travel time) \\
\hline & & & $\min$ \\
\hline & & & $\min$ \\
\hline & & & $\min$ \\
\hline & & & $\min$ \\
\hline
\end{tabular}

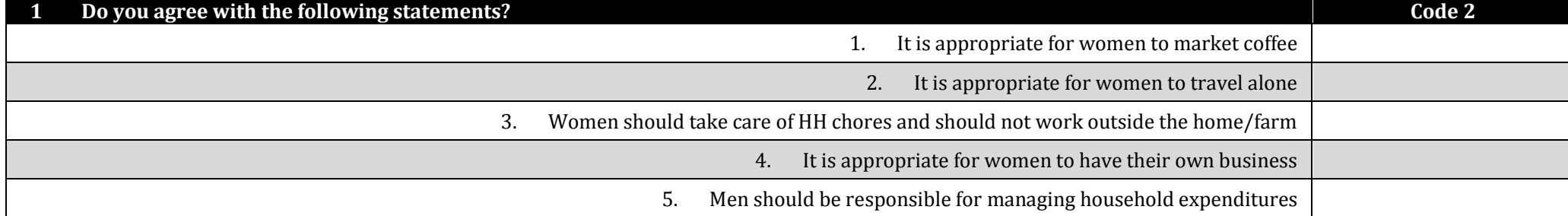




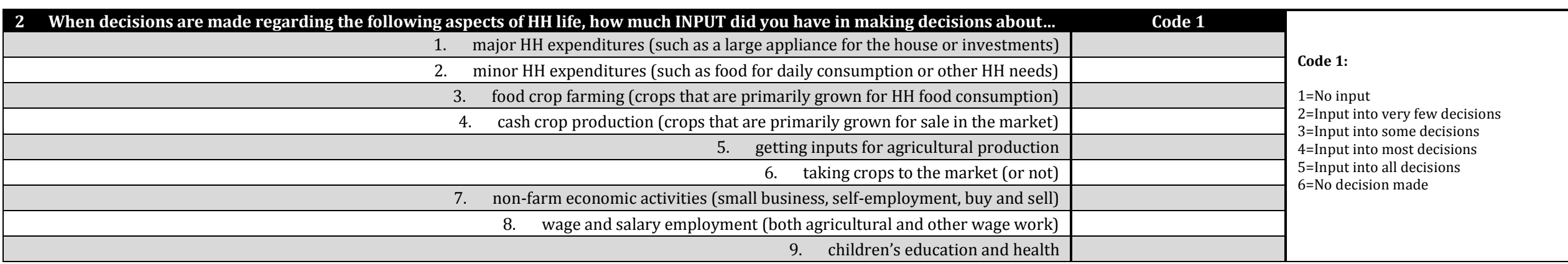

\section{SECTION 19: TIME ALLOCATION}

1 In the last complete 24 hours, starting yesterday morning at 3 am, finishing 2:59 am of the current day, which activities did you carry out? (Intervals are marked in 15 min intervals. Please insert numbers given under code 1 . Ask respondents to narrate their day themselves.)

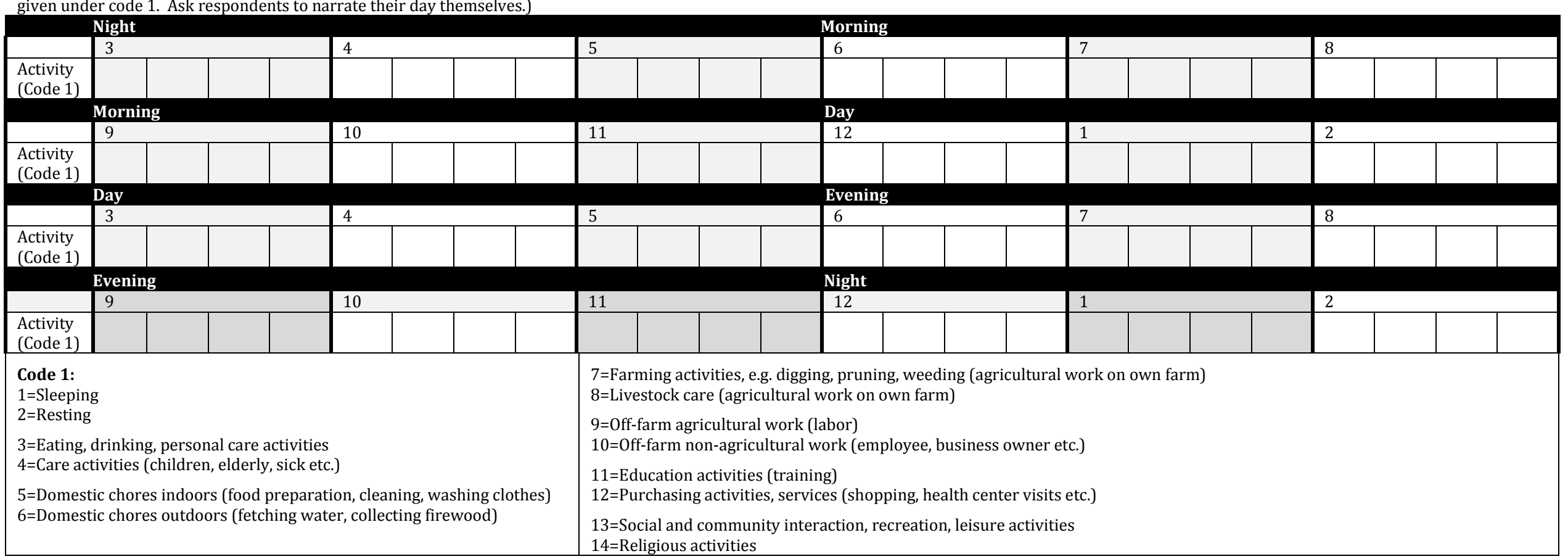

2 How satisfied are you with your available time for leisure activities like visiting neighbors, watching TV, listening to the radio, seeing movies or doing sports?(Code 2 ) Code 2: $1=$ Very satisfied $\quad 2=$ Satisfied $3=$ =Fairly satisfied $\quad 4=$ =Unsatisfied $\quad 5=$ =ery unsatisfied 
Part 2: Gender disaggregated part (Make sure to interview the male and female respondent separately!)

Name of the respondent:

\section{SECTION 15: CHOICE EXPERIMENT}

1.1 Carefully explain the procedure using choice card No. 0 and translate the description of the pictures, Record choices made by the respondent.

\begin{tabular}{|c|c|c|c|c|}
\hline & Choice set number & \multicolumn{3}{|c|}{ Indicate respondent's choice here with a 1 (put 0 for the options not chosen) } \\
\hline Choice set & & & Option 1 & Option 3 \\
\hline Choice set & & & & \\
\hline Choice set & & & & \\
\hline Choice set & & & & \\
\hline Choice set & & & & \\
\hline Choice set & & & & \\
\hline
\end{tabular}

\section{SECTION 16: ATTITUDES TOWARDS CERTIFICATION}

2.0 Is or was this $\mathrm{HH}$ ever certified?

2.1 Do you think your HH benefits from being certified?

2.2 Do you feel you personally benefit from certification?

$(0=$ No $1=Y$ Yes $) \rightarrow$ If no, move to question 3 . $(0=$ No $1=$ Yes $)$

2.3 If yes, what is the GREATEST benefit for YOU? (Code 1)

$(0=$ No $1=$ Yes $)$ projects, being famous) $4=0$ ther (specify)

2.4 Would you say that certification is costly for your HH (e.g. because of required investments)?

3.0 Only ask NON certified respondents that were NEVER certified. $\rightarrow$ If the HH is certified, move to next section.

3.1 Did you ever consider participating in a certification scheme such as UTZ, Fairtrade, Organic?

3.2 Did anyone ever offer you support to become certified (e g farmer organization or NCO)?

3.3 Do you think your HH could benefit from certification?
3.4 Do you think you could personally benefit from certification?

$(0=$ No $1=$ Yes $)$

$(0=$ No $1=$ Yes $)$

3.5 If yes, what would you think would be the GREATEST benefit YOU could derive from certification? (Code 1) $(0=$ No $1=$ Yes $)$

Code 1: $1=$ =Economic benefits (higher price, more income, better market access) $2=$ Training opportunities $3=$ Social benefits (bargaining power, trust among members, collective activities, development projects) $4=0$ ther (specify)

3.6 Do you feel certification would be too expensive for your HH (because it requires certain investments)? $(0=$ No $1=$ Yes $)$

3.7 Would you find it difficult to meet certification requirements such as rules regarding the use of pesticides, prohibition of child and prisoner labor, or coffee quality requirements? $(0=$ No $1=$ Yes $)$ 


\begin{tabular}{|c|c|c|c|c|c|}
\hline \multicolumn{6}{|c|}{ SECTION 17: SOCIAL NETWORKS, ACCESS TO SERVICES (S.I \&S.L) } \\
\hline 1 & Are you a (registered) member of any group? & & $\begin{array}{l}\text { Type of group }{ }^{24} \text { (Code 1) and year membership } \\
\text { started } \\
\text { Group (Code 1):___ since [year]:___ since [year]:___ } \\
\text { Group (Code 1):___ since [year]:__ } \\
\text { Group (Code 1):___ }\end{array}$ & \multirow{2}{*}{\multicolumn{2}{|c|}{$\begin{array}{l}\text { Codes } \\
\text { Code } 1 \\
\text { 1=Farmer organization } \\
\text { 2=Women's group } \\
\text { 3=Credit and savings group } \\
\text { 4=Religious groups } \\
\text { 5=Mutual help or insurance groups (e.g. burial societies) } \\
6=\text { Trade and business associations } \\
\text { 7=Local government groups } \\
\text { 8=0ther (specify) }\end{array}$}} \\
\hline 2 & Is any other HH member a member of any group? & & $\begin{array}{l}\text { PID, type of group }{ }^{22} \text { and year membership started } \\
\text { PID:___Group (Code 1):___ since [year]:_____ } \\
\text { PID:___Group (Code 1):___ since [year]:_____ since [year]:_ }\end{array}$ & & \\
\hline 3 & $\begin{array}{l}\text { Did you hold any leadership position/role in the community within } \\
\text { the last } 3 \text { years? }\end{array}$ & & Role/position (Code 2) & \multirow{2}{*}{$\begin{array}{l}\text { Code 2: } \\
\text { 1=Sub-county chief } \\
\text { 2=Parish chief } \\
\text { 3= Local Council } \\
\text { Committee }\end{array}$} & \multirow{2}{*}{$\begin{array}{l}4=\text { Religious organization } \\
5=\text { Farmer organization/group } \\
6=\text { Extension officer } \\
7=\text { Cultural-spiritual leader } \\
\text { 8=Lead farmer } \\
\text { 88=0thers (specify) }\end{array}$} \\
\hline 4 & $\begin{array}{l}\text { Did any other member of this HH hold any leadership position/role } \\
\text { in the community within the last } 3 \text { years? }\end{array}$ & & Role/position (Code 2) & & \\
\hline 5 & $\begin{array}{l}\text { Are you able to receive credit for agricultural production when you } \\
\text { need such credit? }\end{array}$ & & Source (Code 3) & \multirow{2}{*}{$\begin{array}{l}\text { Code 3: } \\
\text { 1=Farmer group } \\
\text { 2=Cooperative } \\
\text { 4=Local bank } \\
\text { 5=Exporter }\end{array}$} & \multirow{2}{*}{$\begin{array}{l}\text { 6=Local agro dealers } \\
\text { 7=Farmers } \\
\text { 8=Mobile money service providers } \\
\text { 88=0ther (specify) }\end{array}$} \\
\hline 6 & $\begin{array}{l}\text { Has anyone in your } \mathrm{HH} \text { in fact taken any loan or borrowed cash/in- } \\
\text { kind in the past } 12 \text { months? }\end{array}$ & & Source (Code 3) & & \\
\hline 7 & $\begin{array}{l}\text { Who makes the decision about what to do with the borrowed } \\
\text { money/item? (Code } 4 \text { ) }\end{array}$ & & & $\begin{array}{l}\text { Code 4: } \\
1=\text { HHD } \\
2=\text { Spouse }\end{array}$ & $\begin{array}{l}3=\text { Jointly } \\
88=0 \text { ther }\end{array}$ \\
\hline 8 & Do you, personally, (not your HH) have a savings account? & & $\begin{array}{l}\text { Sine when? Year: } \\
\text { With what kind of institution(s)? }\end{array}$ & \multirow{3}{*}{\multicolumn{2}{|c|}{$\begin{array}{l}\text { Code 5: } \\
\text { 1=Formal bank (e.g. Centenary) } \\
\text { 2= Semi-formal financial institution (e.g. MFI, SACCO) } \\
3=\text { Mobile Telephone Operator } \\
\text { 4=Other (specify) }\end{array}$}} \\
\hline 9 & Did you have a savings account in $2012 ?$ & & With what kind of institution(s)? ___ (Code 5) & & \\
\hline 10 & Does any other HH member have a savings account? & & $\begin{array}{l}\text { Sine when? Year: } \\
\text { With what kind of institution(s)? } \\
\text { (Code 5) }\end{array}$ & & \\
\hline
\end{tabular}

5.0 Agricultural training and information

5.1 Are you able to receive agricultural information when you need such information? $(0=$ No $1=$ Yes $)$

5.2 If yes, what is the most important source (Code 5) (allow multiple answers, but START with the most important)

\footnotetext{
${ }^{24}$ If multiple, indicate 3 most important ones.
} 


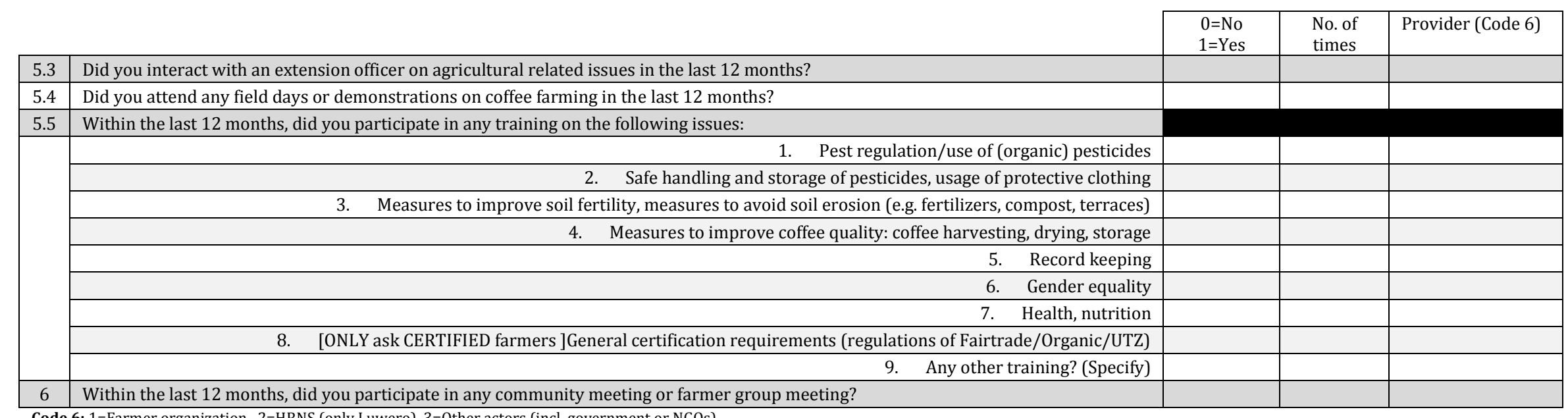

Code 6: 1=Farmer organization 2=HRNS (only Luwero) 3=0ther actors (incl. government or NGOs)

7.0 Speaking in the public

1 Do you feel comfortable speaking up during community or farmer group/organization meetings to share your point

$1 \quad \begin{aligned} & \text { Do you feel } \\ & \text { of view? }\end{aligned}$

2 Do you feel comfortable speaking up in public to protest the misbehavior of authorities or elected officials?

\begin{tabular}{|l|l|}
\cline { 2 - 2 } & (Code 7) \\
\hline
\end{tabular}

Code 7:

$1=$ No, not at all

Yes, but with a great deal of difficulty

Yes, but with little difficulty

$5=$ Yes, very comfortable

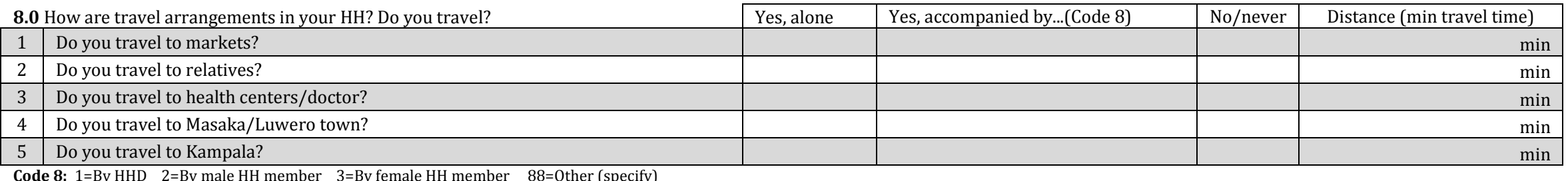

Code 8: 1=By HHD 2=By male HH member $\quad 3=$ By female HH member $\quad$ 88=0ther (specify) 


\section{$1 \quad$ Do you agree with the following statements?}

\section{Code 2}

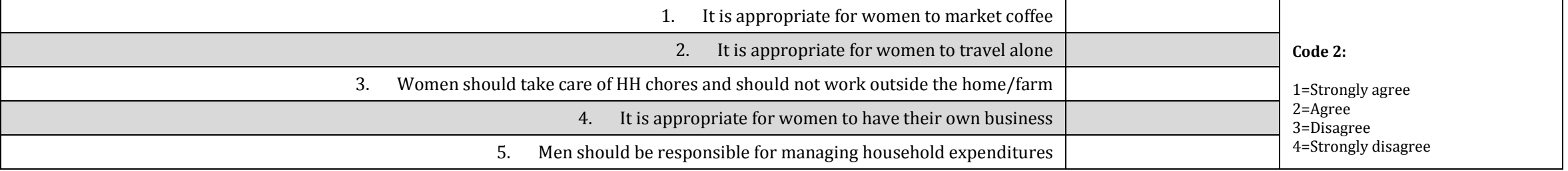

When decisions are made regarding the following aspects of HH life, how much INPUT did you have in making

\section{2 decisions about}

1. major HH expenditures (such as a large appliance for the house or investments)

2. minor HH expenditures (such as food for daily consumption or other HH needs)

3. food crop farming (crops that are primarily grown for $\mathrm{HH}$ food consumption)

4. cash crop production (crops that are primarily grown for sale in the market) 5. getting inputs for agricultural production 6. taking crops to the market (or not)

7. non-farm economic activities (small business, self-employment, buy and sell) 8. wage and salary employment (both agricultural and other wage work)

9. children's education and health

\section{Code 1}

\begin{tabular}{|l|}
\hline \\
\hline \\
\hline \\
\hline \\
\hline
\end{tabular}

Code 1:

$1=$ No input

$2=$ Input into very few decisions

$3=$ Input into some decisions

4=Input into most decisions

$5=$ Input into all decisio

$6=$ No decision made 
SECTION 19: TIME ALLOCATION

1 In the last complete 24 hours, starting yesterday morning at 3 am, finishing 2:59 am of the current day, which activities did you carry out? (Intervals are marked in 15 min intervals. Please insert numbers given under code 1 . Ask respondents to narrate their day themselves.)

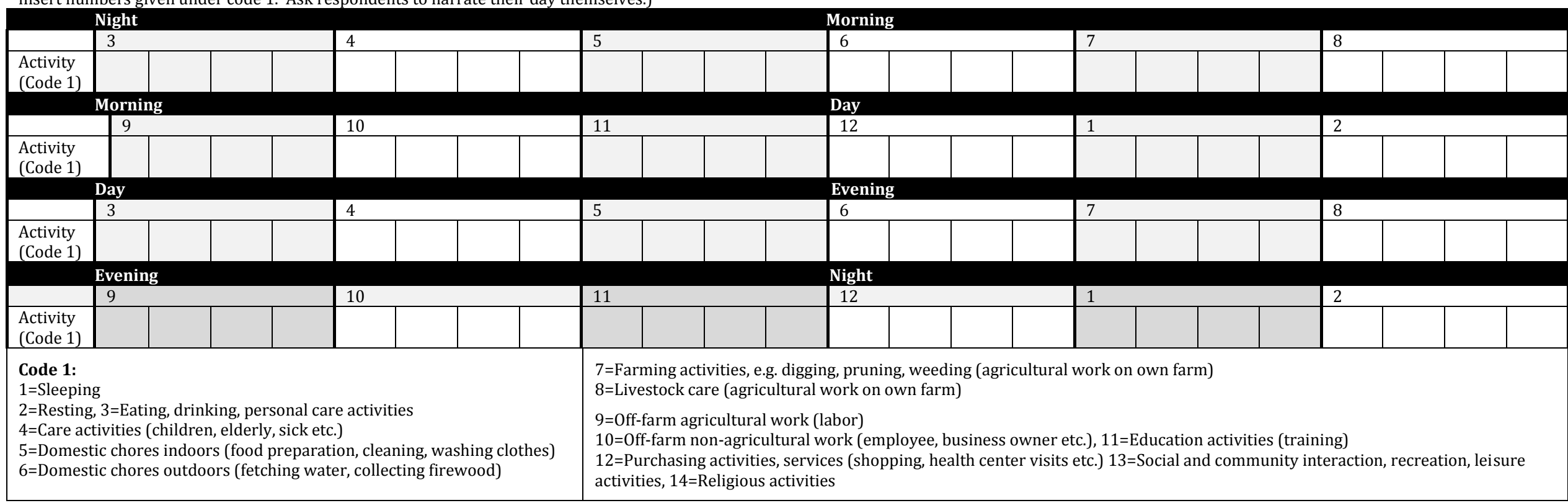

2 How satisfied are you with your available time for leisure activities like visiting neighbors, watching TV, listening to the radio, seeing movies or doing sports?(Code 2) Code 2: $1=$ =ery satisfied $\quad 2=$ Satisfied $3=$ =Fairly satisfied $4=$ Unsatisfied $\quad 5=$ Very unsatisfied 